Technische Fachhochschule Wildau

Fachbereich Betriebswirtschaft/Wirtschaftsinformatik

Studiengang Wirtschaftsinformatik

\title{
Konzeption und Realisierung einer Webseite für die Lateinamerika-Kooperation an der TFH Wildau
}

\author{
Diplomarbeit
}

zur Erlangung des akademischen Grades

Diplom Wirtschaftsinformatikerin (FH)

eingereicht von: Carolina Augspurg

Matrikelnummer: 04277059

eingereicht am: $\quad 1.07 .2008$

Betreuer: $\quad$ Prof. Dr. Christian Müller

Prof. Dr. Ulrike Tippe 


\section{Inhaltsverzeichnis}

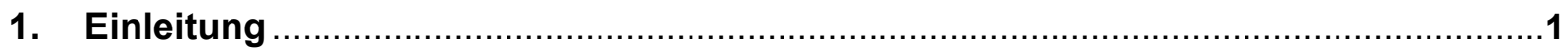

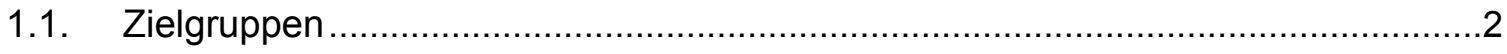

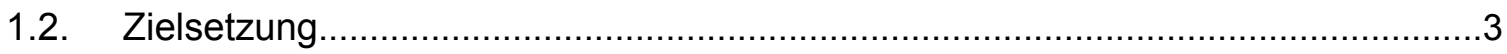

1.2.1. Webseite als Kommunikationsplattform ............................................... 3

1.2.2. Webseite als Marketinginstrument ................................................... 4

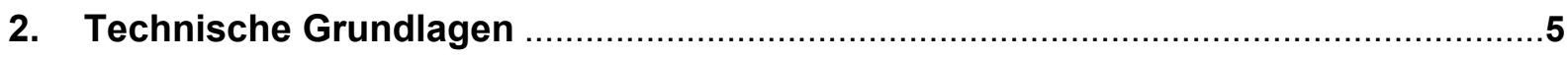

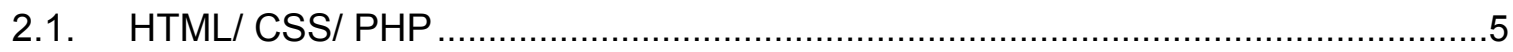

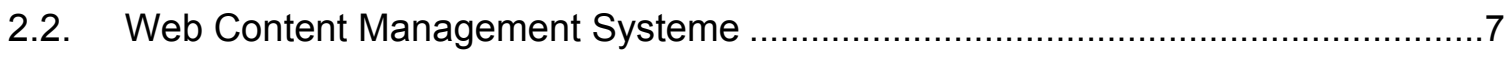

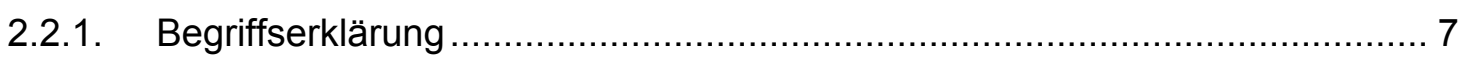

2.2.2. Charakteristische Aspekte von CMS .................................................... 9

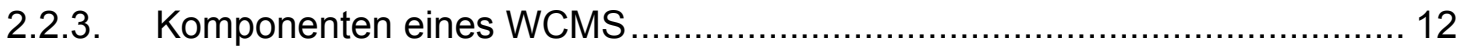

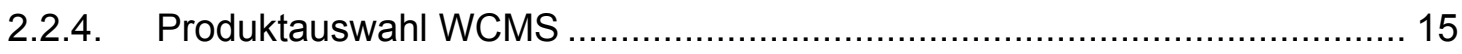

2.3. Herkömmliches Webpublishing vs. Content Management System ......................16

2.3.1. Vor- und Nachteile des herkömmlichen Webpublishing ................................ 16

2.3.2.Stärken und Schwächen von Content Management Systemen....................... 18

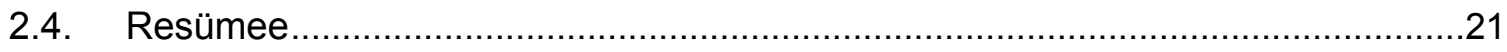

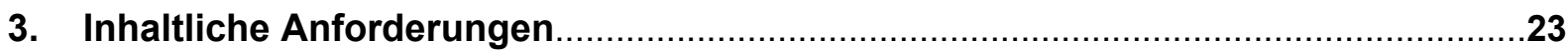

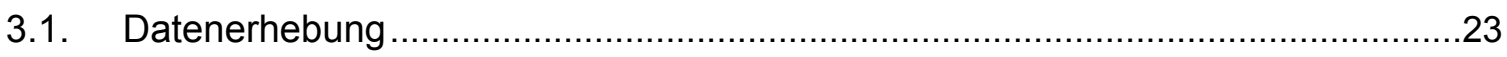

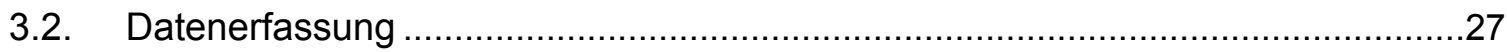

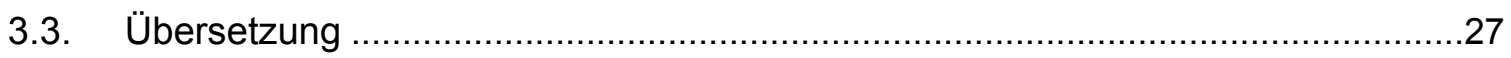

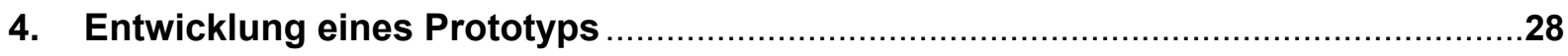

4.1. Erstellung eines Prototyps mit HTML/CSS/PHP .........................................28

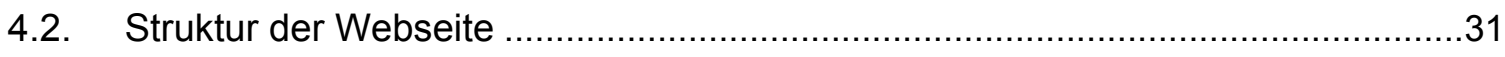




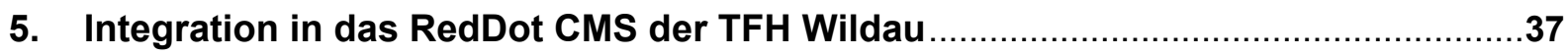

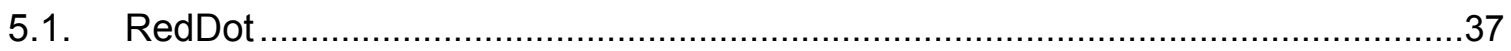

5.2. Einführung der Webseite in das RedDot CMS ..................................................40

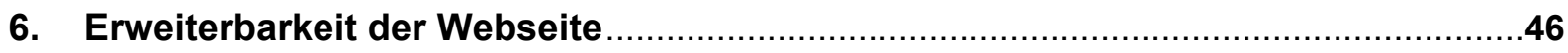

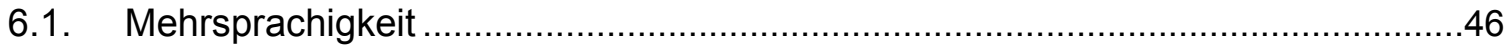

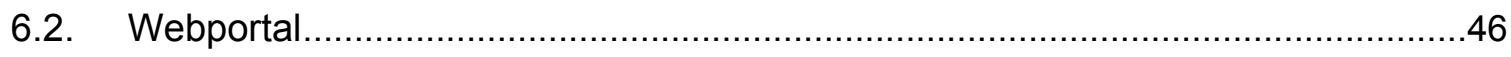

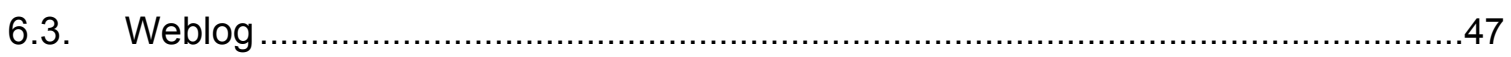

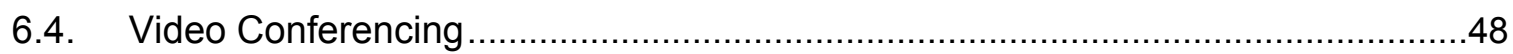

6.5. Ersetzen der „Fachbereiche-Seite“ im Internet-Auftritt der TFH Wildau ...............48

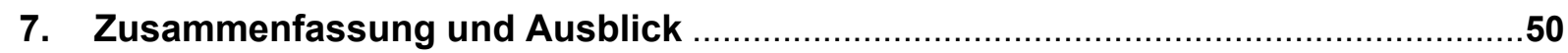

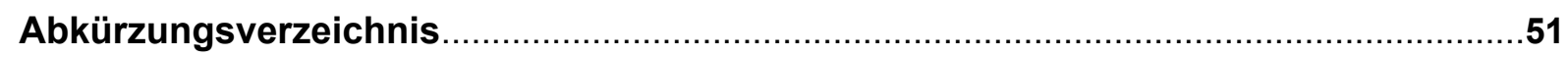

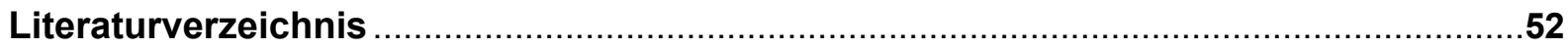

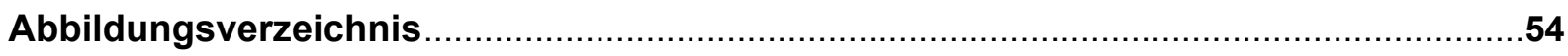

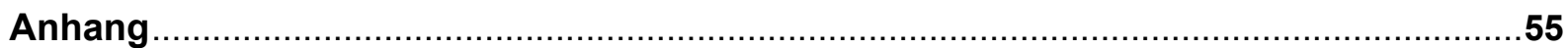




\section{Einleitung}

Auf der Suche nach einem erfolgreichen Konzept zur Erhöhung des Ausländeranteils an der Technischen Fachhochschule Wildau und aufgrund des großen Interesses der lateinamerikanischen Schüler an einem Studium in Deutschland entstand die Idee einer Kooperation zwischen Deutschen Schulen in Lateinamerika und der TFH Wildau. Diese Kooperation besteht nun bereits seit acht Jahren.

Zu Beginn der Kooperation wurde an der Deutschen Schule in Guatemala von einer Studienmöglichkeit an der TFH Wildau berichtet, mittlerweile ist die Anzahl der Kooperationspartner in Lateinamerika gestiegen. Jedes Jahr führt die TFH Wildau eine Informationsreise durch und präsentiert sich in Ländern wie El Salvador, Costa Rica und Kolumbien.

Als Unterstützung für den Kooperationsaustausch wird im Rahmen dieser Diplomarbeit ein Konzept für eine Informations- und Kommunikationsplattform zwischen der TFH Wildau und den lateinamerikanischen Deutschen Schulen entwickelt. Der Einsatz des Internets als Informations- und Kommunikationsplattform bringt eine neue und attraktive Möglichkeit für die TFH Wildau, sich den Kooperationspartnern vorzustellen.

Die vorliegende Diplomarbeit beschreibt die technischen Grundlagen für die Entwicklung einer Webseite mit herkömmlichen Webpublishing Techniken wie HTML, CSS und PHP sowie die Integration der Webseite in das Content Management System der TFH Wildau. Eine Analyse und Gegenüberstellung der Stärken und Schwächen dieser Technologien wird dargestellt.

Es werden inhaltliche Anforderungen an den Internet-Auftritt erörtert, spezifiziert und in einer Prototyp-Implementierung mit HTML, CSS und PHP umgesetzt und erfüllt. Die daraus entwickelte Struktur der Webseite sowie die aufbereiteten Inhalte werden in das Content Management System der TFH Wildau - RedDot CMS - integriert.

Eine weitere Aufgabe dieser Diplomarbeit ist es, Erweiterungsmöglichkeiten in Bezug auf Inhalt und Funktionalität der Webseite aufzuzeigen. 


\subsection{Zielgruppen}

Der folgende Abschnitt analysiert und erörtert die verschiedenen Zielgruppen der Lateinamerika-Webseite.

\section{Lateinamerikanische Schüler und Studenten}

Die Lateinamerika-Kooperation an der Technischen Fachhochschule Wildau richtet sich in erster Linie an Schüler aus Deutschen Schulen in verschiedenen lateinamerikanischen Ländern wie Costa Rica, El Salvador, Guatemala oder Kolumbien.

Diese Schüler haben durch die gründlich erlernte deutsche Sprache und durch ihren Besuch in einer Deutschen Schule bereits eine stärkere Beziehung zu Deutschland als andere Ausländer. Sie bringen aufgrund ihrer Sprachkenntnisse und des hohen Leistungsniveaus die besten Voraussetzungen für ein erfolgreiches Studium in Deutschland mit.

Mit der Webpage sollen Schüler aus lateinamerikanischen Deutschen Schulen und auch deren Eltern innerhalb der Kooperation über Weiterbildungsmöglichkeiten in Deutschland an der TFH Wildau umfassend informiert und aufgeklärt werden.

Neben den Schülern bekommen auch die ehemaligen Schüler, die sich zunächst für ein Studium im Heimatland oder in einem anderen Ausland entschlossen haben, eine Möglichkeit sich zu informieren. Voraussetzung für ein Studium an der TFH Wildau sind gute Kenntnisse in der deutschen Sprache bzw. ein gültiges Deutsches Sprachdiplom Stufe I und Stufe II.

Zu den Zielgruppen dieser Kooperations-Website zählen auch die lateinamerikanischen Studenten der TFH Wildau. Diese Studenten sollen ihre Erfahrungen und Erlebnisse über das Leben und Studieren in Wildau mit lateinamerikanischen Schülern austauschen können.

\section{Deutsche Schüler/Studenten}

Zu weiteren Zielgruppen zählen Schüler, Freunde, Studierende, Dozentinnen und Dozenten, Professorinnen und Professoren sowie alle an Lateinamerika Interessierten. Sie sollen ausführlich über die Kooperation und die Lateinamerika Länder - Chile, Costa Rica, El Salvador, Guatemala, Mexiko, Kolumbien und Venezuela - aufgeklärt werden. 
Es ist bekannt, dass ein Aufklärungsbedarf zu lateinamerikanischen Ländern besteht, da immer wieder von Kommilitonen, Bekannten oder Freunden die Frage gestellt wurde: „Wo liegt eigentlich El Salvador oder Guatemala?".

Informationen über diese Länder z.B. über ihre Traditionen, Lebensweise oder Landschaften, sind zu vermitteln. Die Erstellung von Länderprofilen zielt daher auf einen aktiven kulturellen Austausch und die Bereitstellung von Informationen ab.

\subsection{Zielsetzung}

In diesem Abschnitt werden die Ziele dieser Arbeit definiert. Die Webseite soll einerseits eine Kommunikationsplattform für die lateinamerikanischen Studenten der TFH Wildau und den Schülern, die in Lateinamerika Deutsche Schulen besuchen, darstellen. Anderseits dient die Webseite als Marketinginstrument für die Neugewinnung von Studenten mit lateinamerikanischer Herkunft.

\subsubsection{Webseite als Kommunikationsplattform}

Die Webseite kann die Kommunikation und den Informationsaustausch zwischen Schülern aus lateinamerikanischen Deutschen Schulen und lateinamerikanischen Studenten aus Wildau durch die Publikation und Verwaltung von Inhalten unterstützen. Gleichermaßen soll das Interesse bei deutschen Studenten und Schülern geweckt werden, mehr über lateinamerikanische Länder und deren Kulturen zu erfahren.

Es soll gewährleistet werden, dass Schüler in Lateinamerika die Möglichkeit haben, sich über Kooperationsabkommen zwischen der TFH Wildau und den Kooperationspartnern zu informieren. Die Vorzüge - resultierend aus diesen Kooperationen - finden sich in einer übersichtlichen und verständlichen Darstellung wieder. Darüberhinaus bekommen die Schüler einen Zugang zu Informationen und Inhalten der verschiedenen Fachbereiche und Studienangebote der TFH Wildau. Es wird ein erster Überblick über die Finanzierungs- und Arbeitsmöglichkeiten sowie mögliche Stipendien geboten. Mit der Erstellung von Profilen über lateinamerikanische Studenten in Wildau soll für die Schüler aus lateinamerikanischen Deutschen Schulen eine Übersicht zur Verfügung gestellt werden, welche Studierende welchen Studiengang an der TFH Wildau belegen oder belegt haben. Somit bekommen Schüler eine Gelegenheit, einen ersten Kontakt mit einem oder mehreren Studenten aufzunehmen. 
Eine spezielle Auswahl an Bildern über den Campus der TFH Wildau, den verschiedenen Wohnheimen, den lateinamerikanischen Studenten sowie Sehenswürdigkeiten in Berlin ist zum Vermitteln von Impressionen über das Studieren und Leben in Wildau gedacht. Informationsmaterialen in Form von Berichten und Bildern geben deutschen Interessenten einen Eindruck über lateinamerikanische Länder, deren Kulturen und Traditionen. Um dies zu ermöglichen, werden Länderprofile erstellt, in denen die allgemeinen Grunddaten, Bilder, Überblicke über Universitäten sowie weitere Informationsquellen zu den jeweiligen lateinamerikanischen Ländern dargestellt werden.

\subsubsection{Webseite als Marketinginstrument}

Die Webseite soll als Marketinginstrument dienen und die Möglichkeit bieten, die Technische Fachhochschule Wildau und ihre Angebote und Leistungen weltweit zu präsentieren.

Eine Webseite besitzt allgemein den Vorteil, von jedem und überall abrufbar zu sein. Die auf der Seite veröffentlichten Informationen sind permanent vorhanden, und können so gezielt beworben werden. Diese Informationsplattform kann auf den Homepages der Deutschen Schulen verlinkt werden und somit eine Basis für die jährlichen Besuche bzw. Vorträge über die TFH Wildau in den lateinamerikanischen Ländern bilden.

„Eine ebenfalls unter Marketing-Gesichtspunkten hochinteressante Eigenschaft von einem Internet-Auftritt einer Webseite liegt darin, dass mit Hilfe von Browsern Informationen multimedial präsentiert werden können. “1 Man ist so in der Lage den lateinamerikanischen Schülern aus Deutschen Schulen nicht nur textbasierten Informationen zur Verfügung zu stellen, sondern auch andere Inhalte, z.B. Flyer zu den jeweiligen Studiengängen, Bilder, Videos und Präsentationen zeigen zu können. Der Nutzer kann sich multimedial informieren. Die Vorzüge von einem Studium in Wildau können besser und vielleicht sogar interessanter zum Ausdruck gebracht werden.

Dafür ist es notwendig, die Webseite laufend zu aktualisieren. Neue Bilder oder Berichte über das Studium oder Leben in Wildau verbessern die Informationsqualität der Webseite. Der Internet-Auftritt soll den Nutzer animieren, sich länger auf der Webseite aufzuhalten und den Angeboten zu folgen.

[1] Prof. Dr. H: Klaus Rieder, Dipl.-Volksw. D: Witter-Rieder (2000) 


\section{Technische Grundlagen}

Dieses Kapitel erörtert die technischen Grundlagen für die Entwicklung der LateinamerikaWebseite. Zum einen werden die Ausgangspunkte für eine Prototyp-Entwicklung mit HTML-, CSS- und PHP-Techniken erklärt, anderseits die wesentlichen Grundlagen zum Thema Web Content Management Systeme vermittelt. Dazu erfolgt zunächst eine Definition des Begriffes „Web Content Management Systeme“ (WCMS). Anschließend werden die Merkmale und Komponenten eines WCMS erläutert und eine Marktübersicht der WCMS präsentiert. Der letzte Teil dieses Kapitels behandelt einen Vergleich zwischen herkömmlichen Webpublishing und Content Management Systeme.

\subsection{HTML/ CSS/ PHP}

\section{Hypertext Markup Language - HTML}

HTML ist die Abkürzung für Hypertext Markup Language. Diese Auszeichnungssprache (Markup Language) ist eine Möglichkeit, Textelemente zu kennzeichnen. HTML-Dateien werden im Klartextformat abgespeichert und sind somit software- und plattformunabhängig. Mit HTML können auf diese Weise Inhalte schnell und einfach einer breiten Öffentlichkeit bereitgestellt werden.

HTML-Dateien lassen sich mit fast jedem Textbearbeitungsprogramm editieren und enthalten, neben dem eigentlichen Text, spezifische Befehle, die in Tags stehen. Fast alle Befehle in HTML bestehen aus einem Anfang-Tag und einem Schluss-Tag.

„Eine gewöhnliche HTML-Datei besteht grundsätzlich immer aus einem Header (Kopf), der Angaben zu Titel etc. enthält, und einem Body (Körper), der den eigentlichen Text mit Überschriften, Verweisen und Grafikreferenzen enthält.“2

[2] N. Krugmann (2001) 


\section{Cascading Style Sheets - CSS}

Cascading Style Sheets ist eine Technik, um die Formatierung bzw. das Layout einer HTMLSeite zu verändern. Mit Hilfe von CSS ist es möglich, den Inhalt und das Aussehen einer HTML-Datei von einander zu trennen. Dadurch werden Anpassungen am Layout recht einfach, da nur an zentralen Stellen Daten geändert werden müssen.

CSS legt die Darstellung besonderer Inhalte oder Bereiche innerhalb einer HTML-Seite fest. Neben verschiedenen Angaben zu Farben und Schriften bietet CSS die Möglichkeit Elemente frei zu positionieren oder Hintergrundbilder festzulegen.

„Durch den Einsatz von CSS erreicht man:

- mehr Flexibilität bezüglich der Ausgabemedien,

- Einheitlichkeit in der Präsentation,

- schnelle Aktualisierbarkeit

- Befreiung der Quelldaten von Formatierungsinformationen

- Verkürzung der Ladezeiten der Web-Site“3

Es gibt verschiedene Möglichkeiten, eine CSS-Regel dem HTML-Code zuzuweisen. In einem Stylesheet können die Schrifteigenschaften (Schriftgröße, Schriftart, Schriftfarbe), Linkeigenschaften, Tabelleneigenschaften, Listeneigenschaften u.v.m. in einer externen Datei definiert, gespeichert und über das Element „link“ im „head“ der HTML-Dokumente eingebunden und verknüpft werden.

<link href = "style.css" rel = "stylesheet" type = "text/css" />

\section{Hypertext Preprocessor-PHP}

„PHP ist eine weit verbreitete und für den allgemeinen Gebrauch bestimmte Open Source Skriptsprache, welche speziell für die Webprogrammierung geeignet ist und in HTML eingebettet werden kann." ${ }^{4}$ Der PHP-Code steht zwischen speziellen Anfangs- und Abschluss-Tags (<?php ...?>).

[3] H. Köhler (2008)

[4] PHP: Einleitung - Manual- (2008) 
Ein PHP-Skript wird auf einem Server ausgeführt, auf dem sich die Webseite befindet, d.h. der Quelltext wird nicht an den Browser übermittelt, sondern von einem Interpreter auf dem Webserver interpretiert. Erst die Ausgabe des PHP-Interpreters wird an den Browser gesendet.
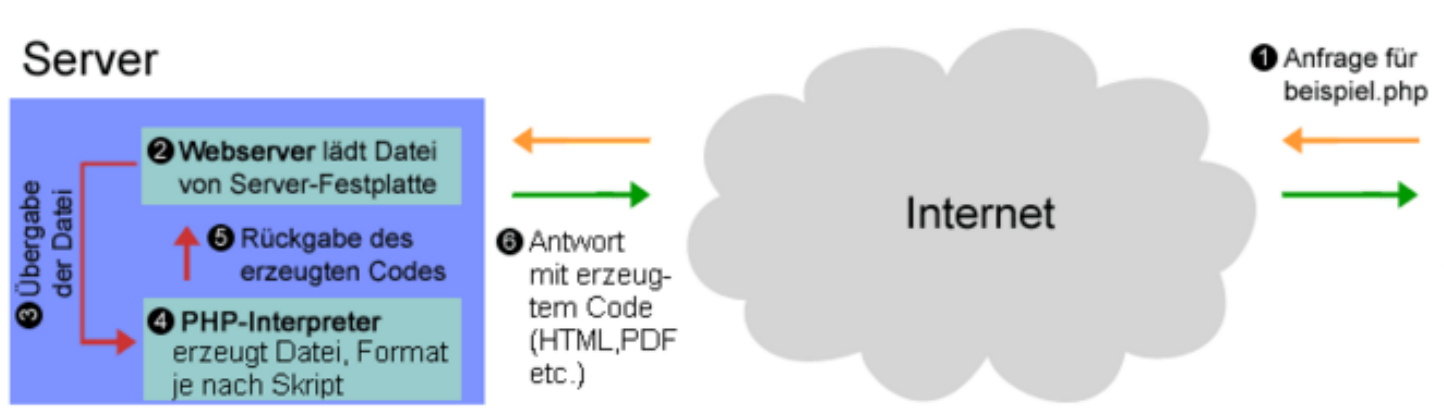

Abbildung 1: Darstellung der Funktionsweise von PHP $^{5}$

Mit PHP können Inhalte dynamisch, d.h. erst bei Aufruf, in eine HTML-Webseite integriert werden.

\subsection{Web Content Management Systeme}

In diesem Kapitel werden die wesentlichen Grundlagen zum Thema Web Content Management Systeme vermittelt. Dazu erfolgt zuerst die Definition des Begriffes „Web Content Management Systeme“ (WCMS). Anschließend werden die Merkmale und Komponenten von einem WCMS aufgelistet und erklärt, sowie der Nutzen, Vor- und Nachteile dargestellt.

\subsubsection{Begriffserklärung}

Um sich dem Thema Web Content Management Systeme zu nähern, werden in diesem Abschnitt die im Zusammenhang stehenden Begriffe erörtert.

[5] S. Brose (2008) 
$\underline{\text { Web }}$

Im Zusammenhang mit dem Begriff WCMS kann „Web“ in allen drei möglichen

Anwendungsformen des Webs - Internet, Intranet und Extranet - genannt werden.

Das Internet ist für die Öffentlichkeit gedacht und ist somit für jeden nahezu unbeschränkt zugänglich. „Das Intranet hat, im Vergleich dazu, eine definierte, meist unternehmensinterne Zielgruppe“6 . Hier haben die Mitarbeiter eines Unternehmens die Möglichkeit Informationen untereinander auszutauschen und verschiedene Anwendungen zu nutzen.

Das Extranet dient der Kommunikation zwischen Unternehmen z.B. mit Kunden, Partnern oder Zulieferern und ist somit nicht der Allgemeinheit, sondern nur ausgewählten Personen innerhalb und außerhalb des Unternehmens zugänglich.

\section{$\underline{\text { Content }}$}

Der Begriff „Content“ kommt aus dem Englischen und bedeutet wörtlich übersetzt Inhalt. Im Zusammenhang mit CMS wird er als „Wissen“, „Information“ und „Dokumente“ verstanden.

Das Online Lexikon „ITWissen“ definiert Content als Sammelbegriff für alle medialen Inhalte. ${ }^{7}$ Content besteht aus einzelnen digitalen Asserts, wie z.B. Bilder, Texte, Grafiken oder Videos.

Erst durch die Verarbeitung von wichtigen Informationen bekommt der Content für einen ausgewählten Nutzerkreis einen entsprechenden Nutzwert.

\section{Content Management}

Der Begriff "Content-Management" wird in Literatur und Praxis allgemein in Verbindung mit der Verwaltung von Webseiten und der Verarbeitung von Informationen für diese verwendet, z.B.: das Erstellen des Layouts, Einfügen der Inhalte, Veröffentlichung der Inhalte und Vergabe von Zugriffsrechten, Publizierung und Archivierung.

„Das Content Management befasst sich mit der systematischen Sammlung, Erstellung, Speicherung und Veredelung von strukturierten Inhalten und Mediendaten aller Art in einem einzigen, fein granulierten (logischen) Bestand. “8

[6] O.Zschau, D. Traub, R. Zahradka (2001)

[7] It-Lexikon (2008)

[8] G. Rothfuss, C.Ried (2001) 


\section{$\underline{\text { Web Content Management Systeme }}$}

Die Umsetzung von Content Management wird von einem Web Content Management System unterstützt und automatisiert. Sie werden für die Beschaffung, Erzeugung, Aufbereitung, Verwaltung und Präsentation von Inhalten (Text, Bilder, Sounds und Videos) im Web (Internet, Intranet oder Extranet) eingesetzt.

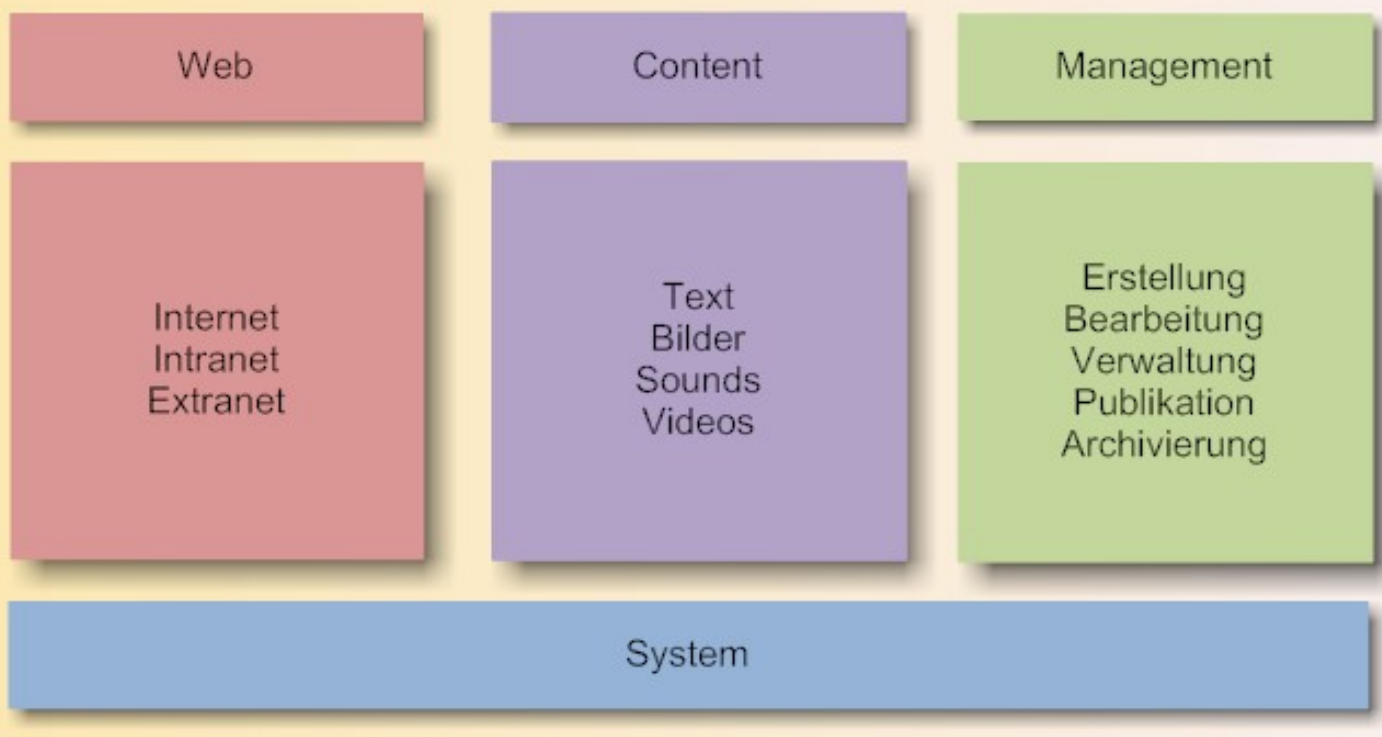

$=$ Web Content Management System

Abbildung 2: Web Content Management System ${ }^{9}$

Solche Systeme basieren auf der Trennung von Inhalt und Layout. Dieses Prinzip erlaubt Autoren ohne HTML-Kenntnisse die Erstellung von Webinhalten in Form von Bildern, Texten oder Links über das Web verarbeiten zu können.

\subsubsection{Charakteristische Aspekte von CMS}

In diesem Abschnitt werden Hauptmerkmale von Content Management Systemen betrachtet.

[9] O.Zschau, D. Traub, R. Zahradka (2001) 


\section{Trennung von Layout und Inhalt}

Ein Hauptmerkmal und Kernprinzip von Content Management Systemen ist die Trennung von Layout und Inhalt, d.h. die Speicherung der Inhalte erfolgt unabhängig von Ausgabeformat und Design.

Die Anordnung und Darstellung der Inhalte basiert auf Vorlagen (Templates). Sie werden durch das Content Management System bei der Generierung einer Webseite automatisch mit Inhalten gefüllt.

Der Inhalt besteht im folgenden Beispiel (Abb. 3) aus einer Überschrift, einer Grafik und einem Fließtext. Die Darstellung wird durch das Template definiert, das die Positionierung der Überschrift, Grafik und Text festlegt. Hier werden außerdem Eigenschaften wie z.B. Schriftfarbe und Schriftgröße bestimmt. Aus diesen Informationen zu Struktur, Inhalt und Darstellung ergibt sich eine fertige HTML-Seite.

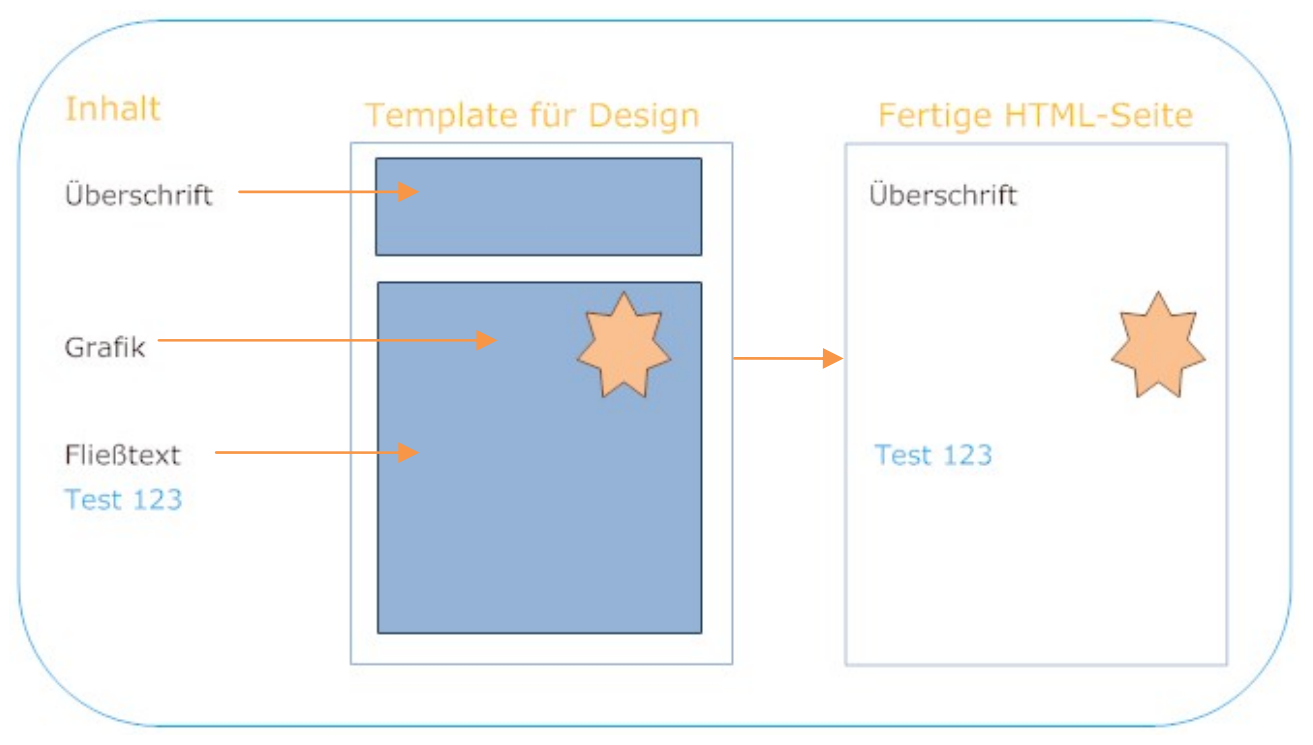

Abbildung 3: Funktionsweise eines Content Management Systems ${ }^{10}$

Mit dem Einsatz von Templates ist es möglich:

- das Layout einer Webseite einzuhalten

- die Verwaltung und Pflege des Contents mit begrenzten Programmierkenntnissen zu gestalten

- Änderungen innerhalb des Layouts ohne großen Aufwand nur mit Anpassungen weniger Templates durchzuführen

- Einhaltung eines Corporate Designs

[10] Jablonski, S.; Meiler, C. (2001) 


\section{Content Life Cycle}

„Ein Web Content Management System verwaltet den Content idealerweise von der Erstellung bis hin zu dessen Generierung oder Entfernung aus dem System.“" ${ }^{11}$ Unabhängig von der Art des Contents (Text, Bild, Video, usw.) folgt es diesem Prozess.

Die Darstellung in Abb. 4 stellt die Reihenfolge der einzelnen Abschnitte innerhalb des Content Life Cycle dar.

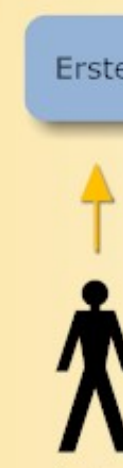

Autor

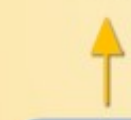

Revision

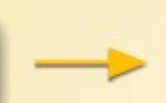

Vorlage
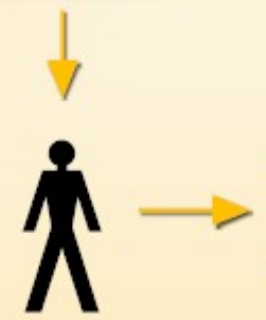

Freigabe

Chefredakteur

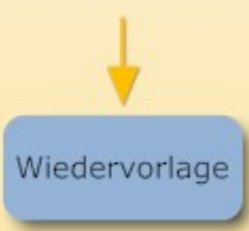

Abbildung 4: Content Life Cycle
Publikation

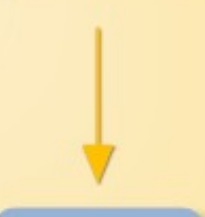

Archivierung

Im ersten Schritt erstellt der Autor den Content. Je nachdem was für ein Content vorliegt, benötigt man verschiedene Tools für die Erstellung, z.B. für einen Text ein Textverarbeitungsprogramm, für HTML einen Editor oder für Fotos ein Bildbearbeitungsprogramm.

Bevor die durch den Autor fertiggestellten Inhalte freigegeben werden dürfen, überprüft diese ein anderer Mitarbeiter (z.B. Chefredakteur).

Ist der Chefredakteur mit den zu veröffentlichen Inhalten zufrieden und einverstanden, werden diese freigegeben und in die Publikationsphase weitergeleitet, ansonsten gehen die Inhalte zum Autor zur Revision und Überarbeitung zurück (Wiedervorlage).

[11] P. Glantsching (2004) 
Freigegebene Inhalte erscheinen publiziert im Inter-, Intra- oder Extranet und sind damit entsprechend öffentlich dargestellt. Der Übergang von der internen Verarbeitung zur externen Veröffentlichung findet in diesem Schritt statt.

Ist der Content nicht mehr aktuell, sollte er aus der Webseite entfernt werden. Dafür findet eine Archivierung der Inhalte statt. „So lassen sich alte Stände der Webseite einsehen, wiederherstellen oder auf Basis alter Beiträge Neue der gleichen Art erstellen, indem die Alten als Muster benutzt werden." ${ }^{2}$

Mit dem Content Life Cycle werden Teamarbeit und Qualitätsmanagement unterstützt. Dadurch, dass der Autor nicht mehr auf einen Techniker angewiesen ist, um seine Inhalte zu publizieren, findet während des Veröffentlichungsprozesses eine Dezentralisierung der Bearbeitung von Content statt.

\subsubsection{Komponenten eines WCMS}

Web Content Management Systeme bestehen aus einzelnen Komponenten:

- Asset-Management

- Workflow-Management

- Benutzer- und Zugriffsverwaltung

- Import- und Exportschnittstellen

\section{Asset-Management}

Das Asset-Management umfasst alle Funktionen, die benötigt werden, den Inhalt einer Seite zu verwalten, zu strukturieren und darzustellen. Hier werden Assets (Text, Grafiken, Videos, usw.) getrennt vom Websitelayout zentral erfasst, verwaltet und gespeichert. Weitere Aufgaben von einem Asset-Management-Tool sind: die Suche, Versionierung und Archivierung von Daten.

[12] O. Zschau (2000) 


\section{Workflow-Management}

„Die Workflow Komponente unterstützt die Abwicklung der Anwendungsprozesse und damit die Einhaltung des Content Life Cycle, welche dem Content Management zu Grunde liegen.“13

Zu den Funktionen einer Workflow-Komponente zählen:

- Benachrichtigungen

- Statusanzeigen

- Freigabezyklen

- Aufgabeverteilungen

- Erstellung von Protokollen

Durch diese Funktionen findet eine Kontrolle und Freigabe von Inhalten statt, was eine inhaltliche Qualität der Webseite sichert.

\section{Benutzer- und Zugriffsverwaltung}

Diese beiden Komponenten, Benutzerverwaltung und Zugriffsverwaltung, arbeiten sehr eng miteinander zusammen.

Die Benutzerverwaltung dient der Zuordnung von Rollen und Berechtigungen für jeden Benutzer, d.h. sie stellt fest, welche Benutzer welchen Content bearbeiten dürfen bzw. müssen.

Die Zugriffsverwaltung schützt die Infrastruktur innerhalb des WCMS vor unerlaubtem Zugriff.

[13] P. Glantsching (2004): 


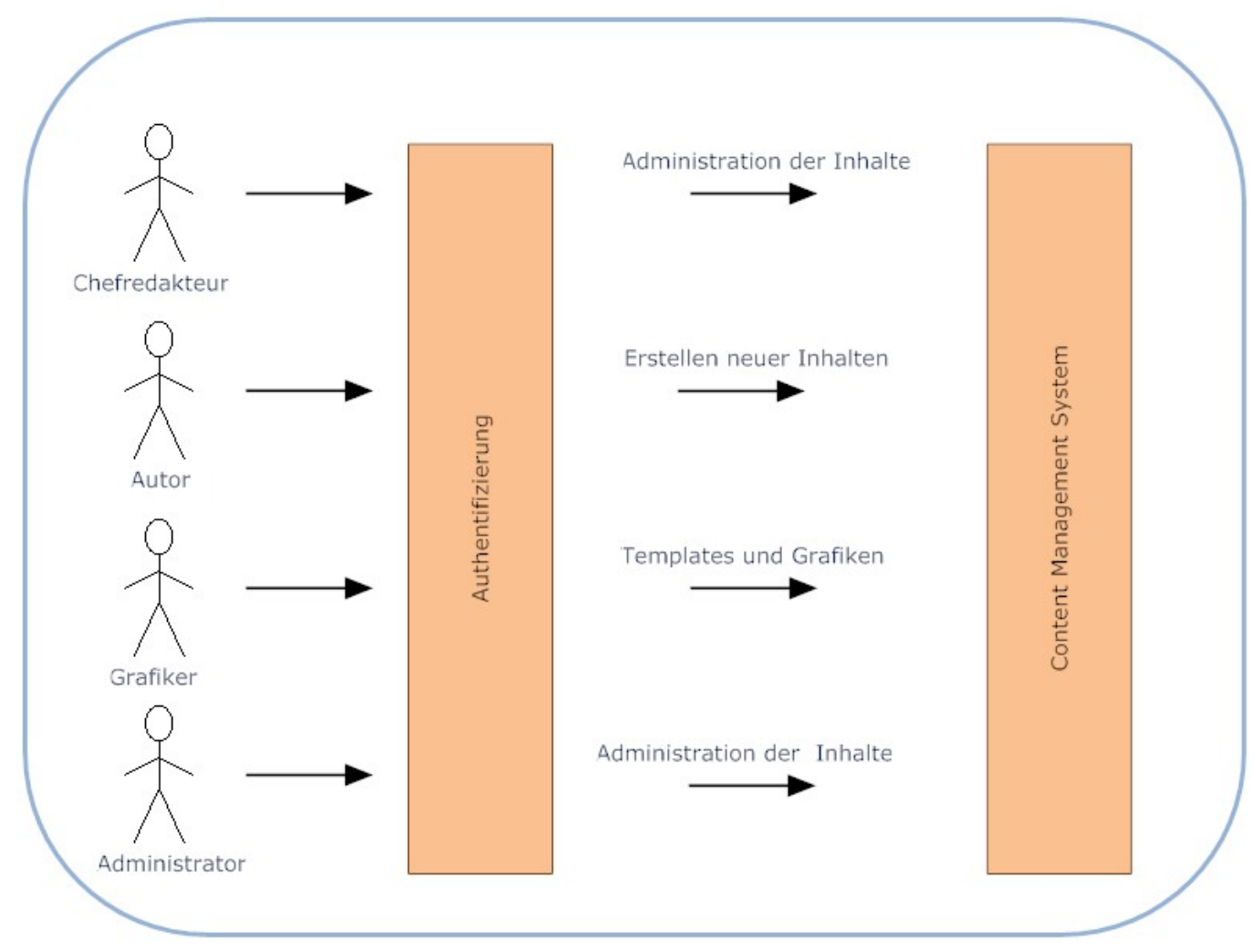

Abbildung 5: Rechte und Rollen im CMS

Bei Web Content Management Systemen erfolgt die Authentifizierung von verschiedenen Benutzern und ihren Rollen, z.B. Chefredakteur, Autor, Grafiker oder Administrator in der Regel über ein Anmeldeformular. Der Administrator erstellt Profile für die verschiedenen Benutzergruppen, in denen festgestellt wird, welche Rechte dem Benutzer zugeteilt sind.

\section{$\underline{\text { Import- und Exportschnittstellen }}$}

Mit der Neu-Einführung von einem WCMS besteht der Bedarf, Content zu im- und exportieren. Über spezielle Schnittstellen können Inhalte in das CMS integriert und weiterverarbeitet (Import) oder bereits existierende Inhalte in andere Systeme exportiert werden. Der XML-Standard - Extensible Markup Language - für den Datenaustausch setzt sich hier immer mehr durch. 


\subsubsection{Produktauswahl WCMS}

Der Markt umfasst ein ziemlich unübersichtliches Angebot an verschiedenen Web Content Management Systemen. Die richtige Wahl für ein geeignetes System bleibt abhängig von den speziellen Bedürfnissen des Unternehmens.

Bei der Klassifizierung von Content Management Systemen kann zwischen verschiedenen Merkmalen unterschieden werden. Die Art der Auslieferung der erstellten Seiten an den Nutzer ist ein Unterscheidungsmerkmal. Diese kann entweder statisch, dynamisch oder hybrid sein.

In der folgenden Tabelle ist eine Auswahl an bekannte Content Management Lösungen mit statischen, dynamischen oder hybriden Eigenschaften dargestellt. Anhand einiger Parameter werden technische Grundeigenschaften dieser fünf CMS gegenübergestellt.

\begin{tabular}{|c|c|c|c|c|c|}
\hline Produktname & FirstSpirit & Joomla! & OpenCms & RedDot & TYPO3 \\
\hline $\begin{array}{l}\text { Produkt- } \\
\text { beschreibung }\end{array}$ & $\begin{array}{l}\text { mächtige und } \\
\text { umfassende } \\
\text { Lösung zur } \\
\text { Verwaltung, } \\
\text { Pflege und } \\
\text { Publikation } \\
\text { sämtlichen } \\
\text { Contents }\end{array}$ & $\begin{array}{l}\text { Leistungs- } \\
\text { fähiges } \\
\text { Open- } \\
\text { Source-CMS, } \\
\text { das einfach } \\
\text { zu warten ist }\end{array}$ & $\begin{array}{l}\text { sehr einfach zu } \\
\text { bedienen und } \\
\text { sorgt für } \\
\text { schnellen } \\
\text { Erfolg beim } \\
\text { Anwender }\end{array}$ & $\begin{array}{l}\text { dient der } \\
\text { Erstellung, } \\
\text { Pflege, } \\
\text { Kontrolle, } \\
\text { Verwaltung } \\
\text { und } \\
\text { Archivierung } \\
\text { von Content }\end{array}$ & $\begin{array}{l}\text { kostenlos } \\
\text { erhältliches } \\
\text { Open Source } \\
\text { Content } \\
\text { Management } \\
\text { System, } \\
\text { zugeschnitten } \\
\text { auf die } \\
\text { Bedürfnisse } \\
\text { von } \\
\text { Unternehmen }\end{array}$ \\
\hline Art Webaufruf & Statisch & $\begin{array}{l}\text { Hybrid } \\
\text { (dynamisch/ } \\
\text { statisch) }\end{array}$ & Dynamisch & Statisch & $\begin{array}{l}\text { Hybrid (Free } \\
\text { Add On) }\end{array}$ \\
\hline Betriebssysteme & Beliebig & Beliebig & Beliebig & Windows & Beliebig \\
\hline Webserver & Beliebig & Apache & IIS, Apache & IIS & Beliebig \\
\hline Datenbanken & Beliebig & MySQL & $\begin{array}{l}\text { MySQL, } \\
\text { Oracle, u.a. }\end{array}$ & $\begin{array}{l}\text { Jede beliebige } \\
\text { ODBC-DB }\end{array}$ & Verschiedene \\
\hline Skriptsprachen & Java & PHP & Java & $\begin{array}{l}\text { beliebig (Perl, } \\
\text { ASP, PHP, ..) }\end{array}$ & PHP \\
\hline Preise & $\begin{array}{l}\text { Server \& } \\
\text { Client } 29.000 \\
€ \\
+ \text { Lizenzpreis }\end{array}$ & $\begin{array}{l}\text { Kostenlos } \\
\text { (Open } \\
\text { Source) }\end{array}$ & $\begin{array}{l}\text { Kostenlos } \\
\text { (Open Source) }\end{array}$ & $\begin{array}{l}\text { Redaktions- } \\
\text { Server } \\
20.000 € \\
\text { Administrator- } \\
\text { Lizenz } 5.000 €\end{array}$ & $\begin{array}{l}\text { Kostenlos } \\
\text { (Open Source) }\end{array}$ \\
\hline
\end{tabular}




\section{Technische Grundlagen \\ Herkömmliches Webpublishing vs. Content Management System

Dynamische Systeme erzeugen die Seiten bei jedem Aufruf neu. Diese haben den Vorteil, dass Veränderungen sofort nach der Freigabe sichtbar werden bzw. dass die Seiten immer aktuell zu sehen sind. Nachteilig ist, dass unter Last es zu einer verzögerten Auslieferung der Seiten kommen kann.

Statische Systeme legen jede Webseite in eine Datenbank oder als Datei ab und sind im Vergleich zu dynamischen Systemen deutlich performanter. Der Seitenaufruf gelingt schneller, da der Webserver nur statische Seiten ausliefern muss. Ein Nachteil ist, dass ein gewisser Zeitraum zwischen dem Erstellen und der tatsächlichen Publikation der Inhalte liegt.

Hybride Systeme kombinieren dynamische und statische Methoden. Sie können somit die Vorteile aus beiden nutzen. Lediglich die Inhalte, die dynamisch aus einer Datenbank generiert werden müssen, wie z.B. News oder personalisierte Inhalte, werden zur Laufzeit erzeugt. Alle anderen Inhalte (Navigation, bestimmte Texte oder Bilder) liegen statisch vor und sorgen damit für eine optimale Performance des Systems.

\subsection{Herkömmliches Webpublishing vs. Content Management System}

In dem folgenden Abschnitt werden die Vor- und Nachteile von herkömmlichem Webpublishing mit den Stärken und Schwächen von Content Management Systemen verglichen.

\subsubsection{Vor- und Nachteile des herkömmlichen Webpublishing}

Ein Nachteil des herkömmlichen Webpublishing ist die mühsame Pflege der Inhalte. Je mehr die Seite wächst, desto schwieriger ist es, den Überblick von z.B. Verlinkungen innerhalb der Seite zu behalten. Jede kleine Veränderung oder Aktualisierung innerhalb der Struktur führt zu hohen Zeitaufwänden, da alles manuell und in den meisten Fällen statisch programmiert wurde.

Werden Strukturveränderungen durchgeführt, muss man große Mengen vorhandener HTMLSeiten editieren (Siehe Abb. 7). Die Entstehung von Inkonsistenzen oder Tote-Links lässt sich kaum vermeiden. 


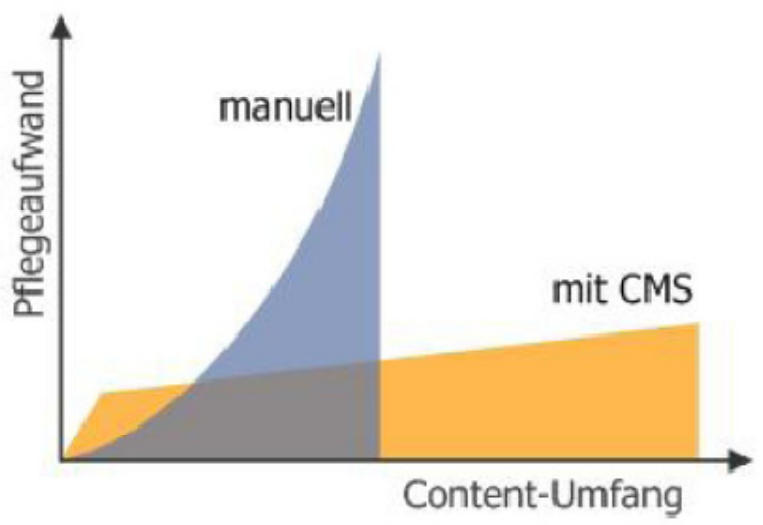

Abbildung 7: Exponentiell wachsender Pflegeaufwand ohne $\mathrm{CMS}^{14}$

Bei dem herkömmlichen Webpublishing liegt die Verantwortung des Publizierens der Inhalte bei einem Techniker. Er administriert den Webserver sowie andere Applikationen und ist meistens für die Veröffentlichung der Inhalte im Web verantwortlich (zentralisierte Pflege der Inhalte).

Jeder Mitarbeiter besitzt eigene Kernkompetenzen in ausgewählten Bereichen, die ihm erlauben mit eingeschränktem Wissen qualitätsvolle Inhalte für die Veröffentlichung zu gestalten. In verschiedenen Unternehmen kommt es oft vor, dass die Mitarbeiter nicht genügend technische Kompetenzen besitzen um Inhalte in das Web zu stellen, d.h. die Inhalte werden nicht von denjenigen publiziert, die diese erstellen.

Der Techniker muss die technische Aufbereitung und HTML-Programmierung der Inhalte übernehmen, obwohl er selber i.d.R. wenig Verständnis für den Inhalt der Seite besitzt. Er ist gezwungen in aufwendiger Koordinationsarbeit die Inhalte aus den Fachabteilungen zusammen zutragen. Die Inhalte müssen meistens in der Darstellung aufbereitet und angepasst werden, damit sie internetkonform publiziert werden können. Daraus ergeben sich Qualitätsverluste, da Mitarbeiterkompetenzen nicht berücksichtig werden. Ohne den Techniker können Informationen nicht veröffentlicht werden, dies führt zu einem „Flaschenhals“ innerhalb des Informationsflusses.

Eine Entwicklung von Webpages mit HTML kann keine effektive dezentralisierte gemeinsame Arbeit von Mitarbeitern mit verschiedenen Kompetenzen anbieten. Aus dieser Situation ergibt sich ein weiteres Problem: die mangelnde Qualitätssicherung. Durch einen fehlenden Workflow besteht die Gefahr, dass Qualitätslücken entstehen. 


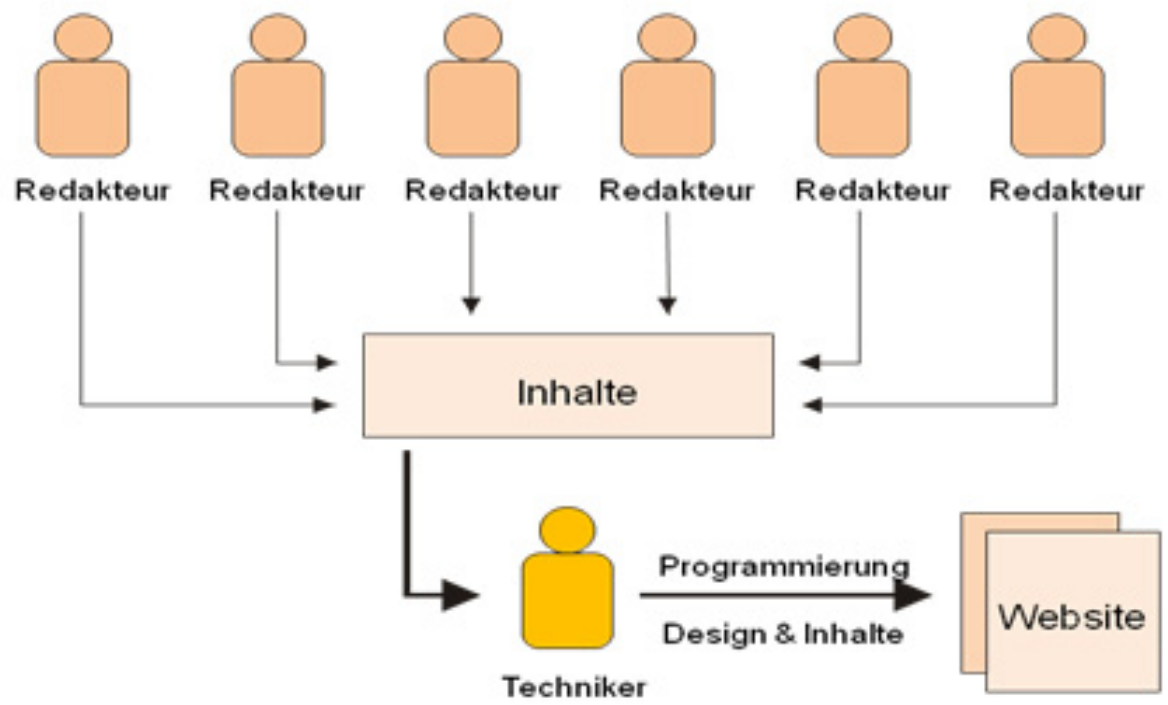

Abbildung 8: Herkömmliches Webpublishing ${ }^{15}$

Trotz der genannten Nachteile einer statischen HTML Webseite bleibt diese immer noch eine schnelle und günstige Variante eine Webpage bzw. einen ersten Lösungsversuch zu erstellen.

Zu den Vorteilen von HTML zählen:

- Die Schreibweise in HTML erfolgt auf eine einfache Art, so dass die Erstellung von (wenigen übersichtlichen) Seiten kein großes Problem darstellt.

- Der HTML-Code besteht aus reinem Text und kann deswegen mit jedem Textbearbeitungsprogramm bearbeitet werden; ein zusätzlicher Editor wird nicht benötigt.

- Da HTML plattformunabhängig ist, kann es in jedem System dargestellt werden.

\subsubsection{Stärken und Schwächen von Content Management Systemen}

Mit der Einführung von einem CMS sollen verschiedene Probleme aus dem herkömmlichen traditionellen Webpublishing gelöst werden. Das Hauptziel von einem Content Management System ist die Anforderungen von dem Content Management zu erfüllen, d.h. große Contentmengen übersichtlich und einheitlich zu organisieren und zu publizieren.

[15] O. Zschau (2000) 
Die Abschnitte aus dem Life Content Cycle (Erstellung, Kontrolle, Freigabe/Wiedervorlage, Publikation und Archivierung) sind automatisiert. Komplette Inhalte werden automatisch archiviert und ganze Navigationsleisten vollständig generiert.

Es wird eine Konsistenz der Links gewährleistet und tote Links vermieden.

Das Einhalten dieses Workflows sichert eine gewisse Qualität und Konsistenz der veröffentlichten Inhalte, da nur kontrollierter Content publiziert wird. Ein erfolgreiches Qualitätsmanagement im Unternehmen ermöglicht schließlich bessere Wettbewerbsfähigkeiten gegenüber der Konkurrenz.
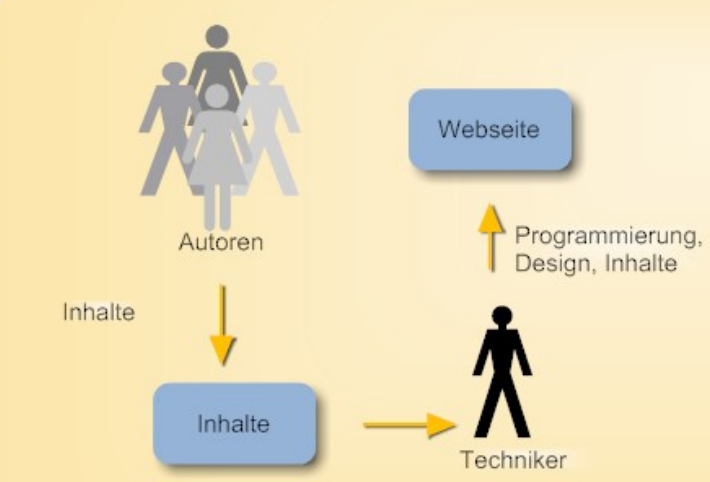

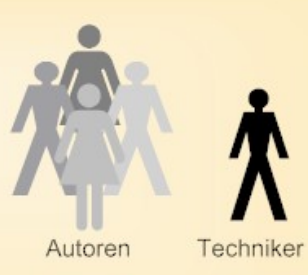

Inhalte

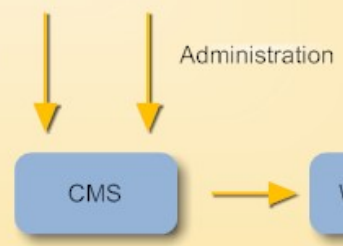

Webseite

Abbildung 9: Webpublishing mit und ohne Content Management Systeme

Die Darstellung in Abb. 9 verdeutlicht den Arbeitsverlauf mit und ohne Content Management System.

Mit dem Einsatz von CMS können die Corporate Identity Vorgaben eines Unternehmens erfüllt werden, da das Layout einer Webseite für alle Unterseiten vorgegeben wird.

Durch die Automatisierung und die Trennung von Inhalt und Layout findet im Vergleich zu traditionellen HTML-Webseiten eine Dezentralisierung der Arbeit statt. Die Autoren können unabhängig von den Technikern selbständig Inhalte erstellen, bearbeiten und letztendlich publizieren. Dank der Seitenbearbeitung im Browser ist man außerdem in der Lage, standortunabhängig zu arbeiten. Es entsteht eine fließende Kette, was die Zeit von der Erstellung bis zur Publikation einer Webseite (Time-to-Web) verkürzt. 
Die einzelnen Bausteine einer Webseite werden mit der Trennung von Inhalt und Layout so zerlegt, dass Mitarbeiter entsprechend ihrer Kernkompetenzen in den

Veröffentlichungsprozess der Webseite eingebunden werden können. So lassen sich Mitarbeiter besser motivieren und an dem Web-Projekt beteiligen.

Steigen beim herkömmlichen Webpublishing die Kosten exponentiell mit dem Umfang der Webseite an, so kann beim Einsatz eines Content Management Systems ein linearer Anstieg erreicht werden. ${ }^{16}$ Das ist realisierbar dank der Zeiteinsparung beim Publizieren und durch die Automatisierung und Dezentralisierung mehrerer Aufgaben (Siehe Abb. 7, S.17).

Die Vorteile und der Nutzen von einem Web Content Management System sind in Abb. 10 dargestellt.

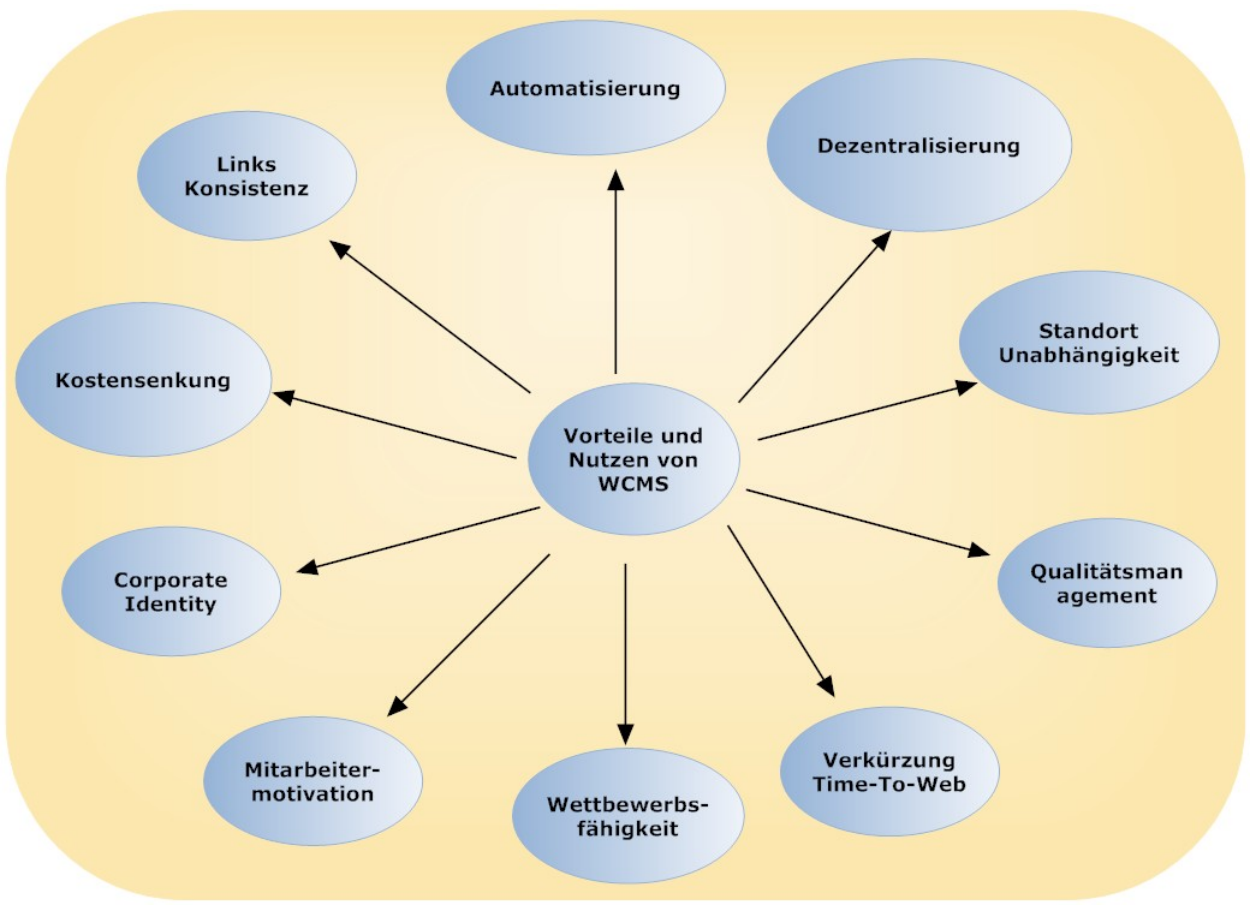

Abbildung 10: Vorteile und Nutzen von WCMS

Die Einführung von Web Content Management Systemen bringt nicht nur Chancen mit sich, sondern kann auch mit Risiken behaftet sein.

Ein WCMS verfehlt schnell sein Ziel, wenn es zu unflexibel entwickelt wurde oder wenn spezifische Anforderungen vom Unternehmen nicht umgesetzt werden können. Die Trennung von Inhalt und Layout verursacht eine (gewollte) Einheitlichkeit der Webseite, was aber auch ein sehr starres, unflexibles Gerüst anlegt. Es besteht die Gefahr, dass zu wenig Freiräume zur individuellen Gestaltung zur Verfügung stehen.

[16] O.Zschau, D. Traub, R. Zahradka (2001) 


\subsection{Resümee}

Man muss sich im Klaren darüber sein, dass die Einführung eines WCMS keine sofortige Kostenersparnis bedeutet, sondern man im Gegenteil am Anfang mit großen Investitionskosten von Software, Server und mögliche Anpassungen rechnen muss. Bei kommerziellen Content Management Systemen können hohe Lizenz- und Supportkosten anfallen. Die Kosten für Open Source CMS begrenzen sich auf Hardware- und Personalkosten für die Umsetzung und Schulungen.

Ohne eine genaue Analyse der Bedürfnisse bzw. der Anforderungen an das System, können sich weitere Schwächen eines CMS bemerkbar machen. Eine ausführliche Untersuchung der Soll-Eigenschaften ist wichtig für eine Produktauswahl.

Ein WCMS ist keine Universallösung für sämtliche Probleme. Eine halbherzige Umsetzung eines CMS kann sich durch schlechte Akzeptanz und Performanceprobleme eher negativ auswirken.

Es gilt die Integration eines CMS in bestehende Abläufe und existierende Infrastruktur erfolgreich umzusetzen. Eine Anpassung an die organisatorischen Abwicklungen ist dabei meistens notwendig.

Der Einsatz von einem Web Content Management System bedeutet nicht, dass der Content sich jetzt selbständig aktualisiert. Die Kombination aus aktuellen und interessanten Informationen ist ein Teil des Erfolges vom Gesamtsystem. Die Inhalte der Seite regelmäßig zu aktualisieren, bleibt immer noch Aufgabe des Menschen.

Die Grenzen von Web Content Management Systemen sind in Abb. 11 zusammengefasst. 


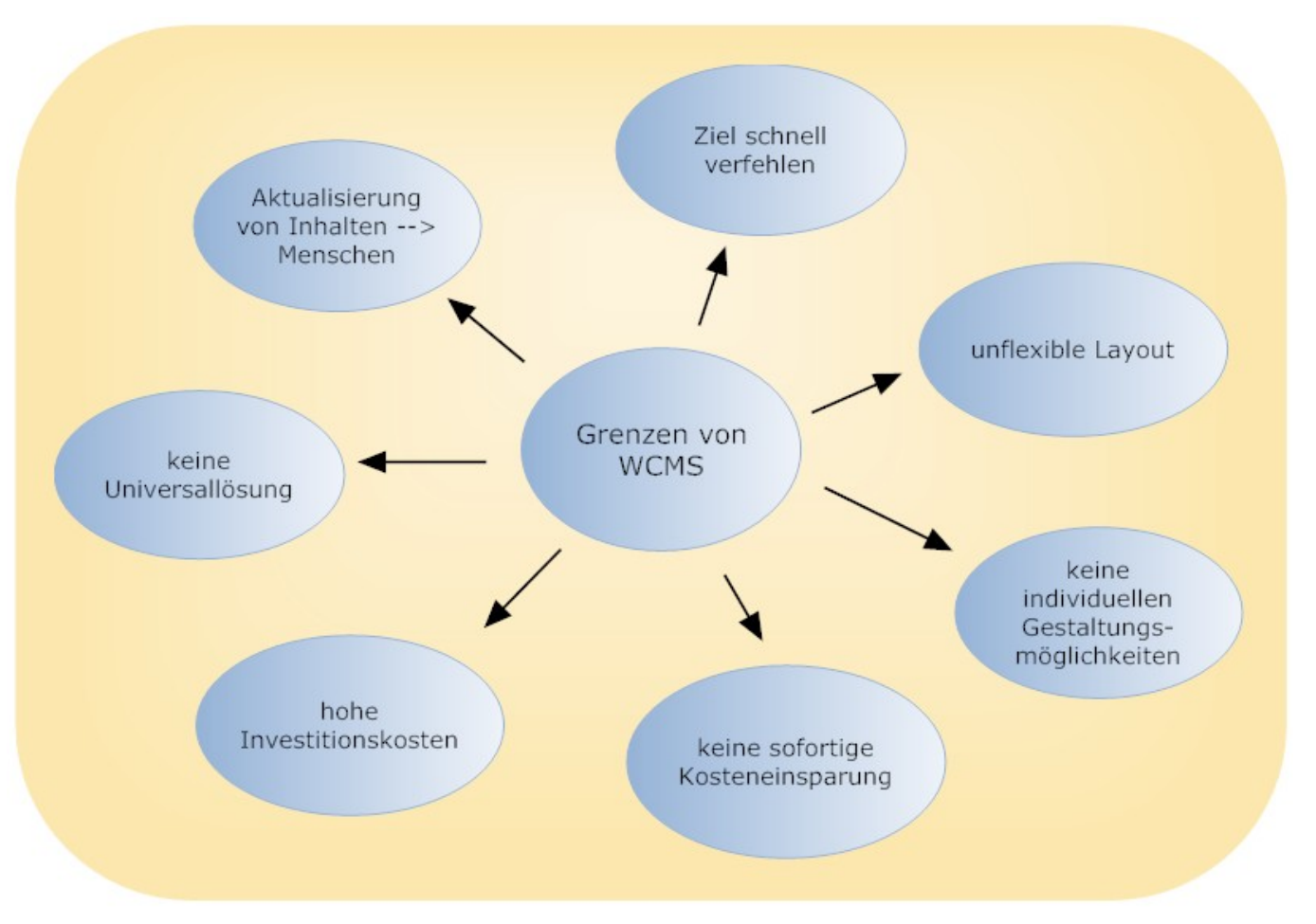

Abbildung 11: Grenzen von WCSM

Nichts desto trotz stellen die Vorteile eines CMS eine Möglichkeit dar, den Problemen des herkömmlichen Webpublishing zu begegnen. Die Einführung eines Content Management erlaubt ein direktes Publizieren der Inhalte, auch für Mitarbeiter ohne HTML-Kenntnisse. Dabei werden die Kernkompetenzen jedes Mitarbeiters berücksichtigt und man ist in der Lage dezentralisierter zu arbeiten. Durch den automatisierten Workflow ist einer Qualitätsund Konsistenzsicherung der Inhalte gewährleistet, da nur kontrollierter Inhalt publiziert wird. Änderungen im Layout und in der Struktur der Webseite sind durch eine einfache Anpassung der Vorlagen realisierbar.

Allgemein gilt: Je größer ein Webprojekt ist, desto eher sollte man bei der Planung den Einsatz eines WCMS überdenken. 


\section{Inhaltliche Anforderungen}

In Zusammenarbeit mit Prof. Dr. Christian Müller werden die inhaltlichen Kriterien der Webseite abgestimmt. Die erforderlichen Daten für die Profilerstellung der jeweiligen Studenten und Länder, die Beschreibung von Finanzierungsmöglichkeiten und die Bilder für eine Galerie, müssen erhoben, erfasst und aufbereitet werden.

\subsection{Datenerhebung}

„Die Erhebung ist ein Prozess zur Bereitstellung von Daten. Dabei ist es unerheblich, in welcher Form dies geschieht, also z.B. (fern)mündlich, schriftlich oder elektronisch. Die zu erhebenden Daten existieren zwar, stehen aber nicht unmittelbar zur Verfügung. “17

Es bestehen unterschiedliche Wege, die verschiedenen Methoden und Verfahren zur Datenerhebung zu systematisieren. Häufig jedoch ist die Unterscheidung zwischen Primärund Sekundärerhebung zu erkennen.

„Bei der Primärerhebung werden Daten eigens für den Untersuchungszweck erhoben. Sekundärerhebungen werden auf Grundlage von unternehmensexternen oder für andere unternehmensinterne Zwecke erhobenen Daten durchgeführt. “18

Methoden der Primärerhebung unterscheiden sich in Befragung und Beobachtung. Zu den Sekundärerhebungsmethoden zählen die Inventurmethode, die Dokumentenanalyse und der Unternehmensvergleich (Siehe Abb. 12, S. 24).

Bei den Datenerhebungen für die Webseite ist die Methode der Primärerhebung (Befragungen) anzuwenden. „Ziel ist es, von den Befragten die gewünschten, zielgerichteten Informationen zu erhalten."19

Die benötigten Daten für den Inhalt der Webseite sollen von lateinamerikanischen Studenten in Form von mündlichen Interviews und per E-Mailverkehr elektronisch erhoben werden. Am Ende der Erhebung stehen die Daten zur Verfügung, die entweder schon aufbereitet sind oder erst den Prozess der Datenerfassung durchlaufen müssen.

[17] K. Hömberg, D. Jodin, M. Leppin (2004)

[18] K. Hömberg, D. Jodin, M. Leppin (2004)

[19] S. Broda (2005): 


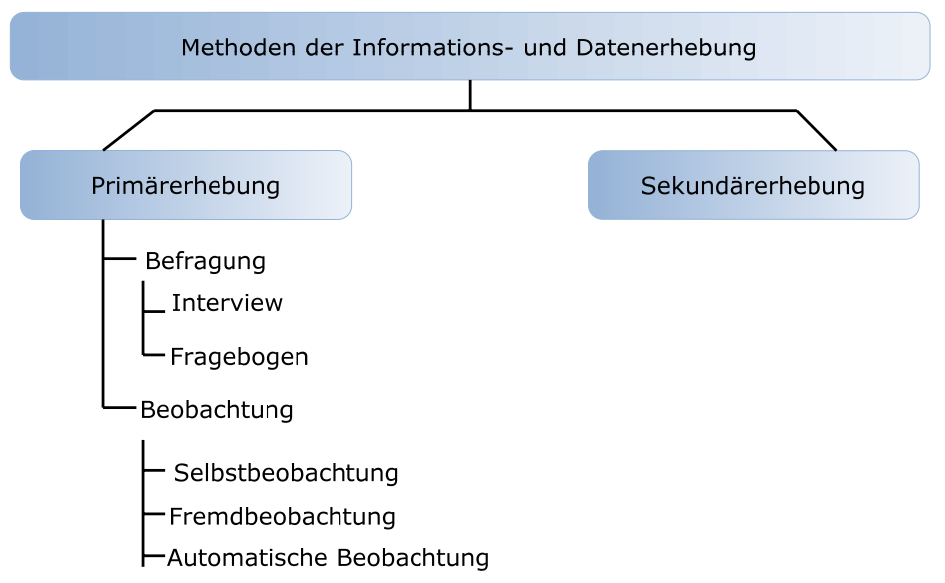

Abbildung 12: Methoden der Informations- und Datenerhebung

\section{Befragungen zu den Profilen}

Eines der wesentlichen Ziele dieser Kooperation und somit der Webseite ist es, den Informationsaustausch zwischen lateinamerikanischen Schülern von Deutschen Schulen und lateinamerikanische Studenten an der TFH Wildau anzuregen und zu vereinfachen. Um eine erste Kommunikation bzw. Kontaktmöglichkeit zwischen den Schülern und den Studenten zu ermöglichen, sollen Profile von und über die Studenten erstellt werden.

In den Profilen sollen folgende Informationen von den Studenten enthalten sein:

- Persönliche Angaben
- Name
- Alter
○ E-Mail-Adresse

- Studiengang

- Studiengangbeschreibung

- Angaben zum Studienbeginn

- Semesterstand

- TFH Wildau

- Bericht über das Leben in Wildau/Studium in Wildau

- Bild

Als Kommunikationsmittel für die Datenerhebung sind hier E-Mails zu bevorzugen, da die Daten so bereits in digitaler Form vorliegen und weiterverarbeitet werden können. 


\section{Befragungen zu Finanzierungsmöglichkeiten}

Ein wichtiger Aspekt bei der Auswahl eines Auslandstudiums sind u.a. die anfallenden Kosten. Gerade in lateinamerikanischen Ländern muss man davon ausgehen, dass nicht alle Familien über ausreichende finanzielle Mittel verfügen, um ihren Kindern eine Ausbildung im Ausland zu ermöglichen. Viele entscheiden sich aus diesem Grund nicht ins Ausland zu gehen und in ihren Heimatländern zu studieren.

Um dem entgegen zu wirken und mit dem Ziel die Anzahl der lateinamerikanischen Studenten an der TFH Wildau zu vergrößern ist es für diese Kooperation wichtig, auf der Webseite über Finanzierungsmöglichkeiten während eines Studiums für einen aus Lateinamerika stammenden Schüler zu informieren.

Dazu muss eine allgemeine Recherche über existierende Finanzierungs- und Arbeitsmöglichkeiten für Ausländer in Deutschland durchgeführt werden. Nachdem diese definiert werden, erfolgen Untersuchungen, welche der lateinamerikanischen Studenten an der TFH Wildau:

- als studentische Hilfskraft an der TFH Wildau arbeiten

- als studentische Mitarbeiter außerhalb der TFH Wildau arbeiteten

- einen Nebenjob ausüben

- über Praktikumserfahrungen verfügen

- ein Stipendium bekommen.

Nach einer Einteilung der Studenten in die o.g. Gruppen, kann man die Studenten nach spezifischen Angaben und Erfahrungen mündlich oder schriftlich per Email befragen. Ausgewählte Studenten innerhalb jeder Gruppe (1-2 pro Gruppe) nehmen an der Umfrage teil.

In Interviews mit Studenten soll ermittelt und hervorgehoben werden, wie der Bewerbungsablauf als Studentische Hilfskraft, für einen Nebenjob oder bei einem Praktikumsangebot ist. Als ebenfalls wissenswert gelten Voraussetzungen für den Erhalt eines Stipendiums. 


\section{$\underline{\text { Umfrage zu den Grunddaten der Heimatländer }}$}

Eine Darstellung der verschiedenen lateinamerikanischen Länder auf der Webseite ist wichtig, um Zielgruppen zu informieren und anzusprechen.

Mit Profilen über lateinamerikanische Länder soll insbesondere die deutsche Zielgruppe erreicht werden. Deutsche Interessenten finden mit dieser Webseite grundlegende Informationen zu Lateinamerika.

Die Länderprofile bieten folgende Rubriken:

- Landesinformationen

- Landkarte

- Weitere Quellen

- Bilder

- Weitere Bilder und touristische Informationen

- Universitäten

Die Inhalte zu den Rubriken Landesinformationen, Landkarte, Weitere Quellen, Weitere Bilder und touristische Informationen sollen recherchiert und für die Veröffentlichung aufbereitet werden. Studenten selbst sollen einige länderspezifische Informationen bereitstellen.

Die folgende Tabelle gibt einen kurzen Überblick über den aktuellen Anteil der Studierenden aus den jeweiligen Herkunftsländern.

\begin{tabular}{cc}
\hline Herkunftsland & Anzahl der Studenten \\
\hline Chile & 1 \\
\hline Costa Rica & 1 \\
Guatemala & 12 \\
\hline El Salvador & 8 \\
Kolumbien & 1 \\
\hline Mexiko & 1 \\
\hline Venezuela & 1 \\
\hline Abbildung 13: Lateinamerikanische Studenten an der TFH Wildau
\end{tabular}

Für die Sammlung der Daten der lateinamerikanischen Partnerländer sollen die Studenten mittels E-Mail Nachrichten angesprochen werden.

Es werden Bilder zu den entsprechenden Herkunftsländern und eine Liste über bekannte Universitäten in jedem Land angefordert. Eine Antwort ist per E-Mail vorgesehen. Jedes Land wird dann gemäß den erhaltenen Informationen in einem Profil vorgestellt. 


\section{Umfrage zu Bildern}

Es ist vorgesehen, in dem Webprojekt einen zusätzlichen Bereich in der Seitenstruktur anzulegen. Dieser Bereich soll die Bilder aus Wildau und seiner Umgebung, Berlin und seine Sehenswürdigkeiten und den Campus und die Labore der TFH Wildau enthalten. Die auf diesen Seiten dargebotenen Fotos stellen die Studenten bereit.

\subsection{Datenerfassung}

Nach der Erhebung der Daten durch Befragungen und Interviews, müssen diese erfasst bzw. aufbereitet werden. „Die Datenerfassung bezeichnet alle manuellen und automatischen Arbeitsvorgänge, mit denen anfallende Daten in eine maschinenlesbare Form gebracht werden. “20

Die Daten aus den Interviews mit den Studenten müssen durch Aussortierungen und Bewertungen analysiert und strukturiert werden. Nach Auswertung und Analyse der erhaltenden Informationen soll es möglich sein, diese in eine einheitliche Darstellungsform zu bringen und als Bestandteil der Webseite zu veröffentlichen.

\section{3. Übersetzung}

Der erste Schritt der Webseiten-Entwicklung ist eine Anfertigung einer deutschsprachigen Version der Webseite. Eine Übersetzung der Seite in die Fremdsprache Spanisch ist bereits während den ersten Projektbesprechungen als Hauptanforderung an die Webseite definiert worden. Die Übersetzungsarbeiten sollen demzufolge bei der Erstellung des ersten Prototyps der Webseite beginnen und parallel zu Entwicklungstätigkeiten ausgeführt werden.

Um den Übersetzungsaufwand zu reduzieren, wird schon bei der Datenerhebung darauf hingewiesen, dass die Profilinformationen der Studenten möglichst zweisprachig (deutsch und spanisch) anzufertigen sind. Eine Übersetzung von Deutsch auf Spanisch ist von besonderer Bedeutung, denn nur so kann sichergestellt werden, dass alle Zielgruppen der Kooperation angesprochen werden. Die Informationen sollen nicht ausschließlich für die lateinamerikanischen Schüler (deutschsprachig) zugänglich sein, sondern auch für deren Eltern, die meistens nicht die deutsche Sprache beherrschen.

[20] H. Engesser, V. Claus, A. Schwill (1993): 


\section{Entwicklung eines Prototyps}

Dieses Kapitel verdeutlicht die Struktur und die Entwicklung eines Rapid Prototyps in HTML mithilfe von CSS und PHP der Lateinamerika Webseite.

\subsection{Erstellung eines Prototyps mit HTML/CSS/PHP}

Es besteht die Anforderung, schnell einen ersten lauffähigen Prototyp für das KooperationsWebprojekt zu erstellen. Dafür wird das Konzept einer statischen Webseite entwickelt, was einen schnellen, zu ersten Ergebnissen führenden Lösungsansatz des Projektes bietet. Dieser wird mit Programmiertechniken wie CSS und PHP unterstützt.

Die erforderlichen Daten wurden von den lateinamerikanischen Studenten in schriftlicher und digitaler Form oder mündlich übermittelt, anschließend erfasst und in eine einheitliche Form gebracht.

Studenten der TFH Wildau erstellten dazu u.a. Berichte für die Veröffentlichung auf der Webseite. Prof. Dr. Christian Müller, Verantwortlicher für die Lateinamerika-Kooperation an der TFH Wildau, und von Prof. Dr. Lázló Ungvári, Präsident der TFH Wildau, stellten zusätzlich Informationen bereit. Trotzdem blieben zahlreiche inhaltliche Fragen offen, die im Laufe der Zeit eigenständig recherchiert wurden.

Die Webseite konnte von Deutsch auf Spanisch erfolgreich übersetzt werden. Dabei stellte die Studiengänge-Seite den größten Zeitaufwand dar, da diese fachliche und wissenschaftliche Begriffe zu jedem Studiengang beinhaltet.

Nachdem die Daten vollständig erfasst und aufbereitet vorlagen, wurde ein Prototyp der Webseite entworfen und entwickelt. Für die Implementierung dieser Seite waren zahlreiche HTML-Dokumente zu erstellen. Diese wurden mit einer geeigneten Verzeichnisstruktur systematisiert und abgelegt: Deutsche HTML-Dateien in den Ordner „DEU“, Spanische in „ESP“. Im Verzeichnis „images“ sind sämtliche Bilder gespeichert. Beide Webseiten, die deutsche und die spanische Version, greifen auf den Ordner „images“ und damit auf dieselben medialen Dokumente zu. Eine redundante Speicherung wird so umgangen. 
Im Laufe der Entwicklung des Prototyps zeigte sich jedoch, dass der Umfang der inhaltlichen und technischen Anforderungen der Webseite stetig anwuchs. Strukturelle Änderungen innerhalb der Webseite sowie Änderungen der Navigation bedeuteten große Aufwände und waren nicht in kurzer Zeit realisierbar. Das Hinzufügen oder Löschen von Links sowie jede andere Strukturveränderung der Webseite, was während der Entwicklungsphase relativ oft vorkam, wurde durch die statische Programmierung zu einer großen Herausforderung.

Mit Programmiertechniken wie CSS und PHP wurden erste dynamische Teile in das statische HTML integriert und so der Änderungsaufwand - resultierend aus aktualisierten Anforderungen - minimiert.

Es wurde eine externe Datei (style.css) geschrieben und in den HTML-Code eingebunden.

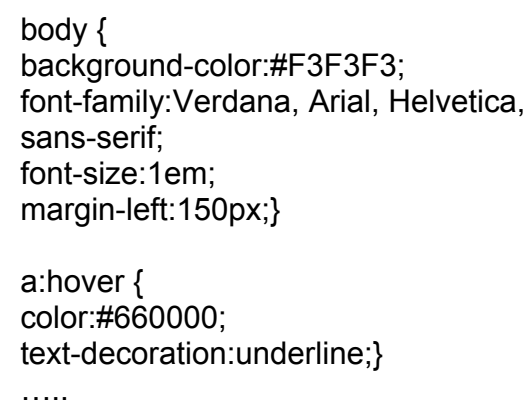

Abbildung 14: Ausschnitt von style.css

\section{Erläuterung zur Abbildung:}

Diese Abbildung zeigt einen kleinen Ausschnitt aus dem Stylesheet „style.css“ und legt Eigenschaften für den Körper und für die speziellen Linkeigenschaften fest.

Die Körper-Eigenschaften (body) beziehen sich auf den gesamten sichtbaren Körper der HTML-Datei und beschreiben hierfür die Hintergrundfarbe (background-color:\#F3F3F3), die Schriftart (font-family:Verdana, Arial, Helvetica, sans-serif), die Schriftgröße (font-size:1em) sowie den Abstand auf der linken Seite der Webseite.

„A:hover“ definiert die Link-Eigenschaften, wenn der Anwender mit der Maus darüber fährt. Hier wird die Linkfarbe (color:\#660000) gewechselt und der Text unterstrichen (textdecoration:underline)

CSS ersparte enorm Zeit bei Anpassungen und Veränderungen von den verschiedenen Formatierungen. In dieser Datei wurden alle Gestaltungseigenschaften der Webpage definiert und konnten somit zentral mit wenigen Eingriffen editiert werden. 
Trotzdem gab es Verbesserungspotential bei der Gestaltung dieses Prototyps. Einen großen Aufwand stellten Veränderungen des Navigationsmenüs dar.

Während der Projektentwicklungsphase wurde ein PHP-Skript geschrieben (inc.navi.php). mit der Funktion function printnavi(), um damit das Navigationsmenü der Webseite dynamisch einzubetten.

Die Navigationsleiste in den HTML-Seiten wurde durch die PHP-Funktion <?php printnavi();?> ersetzt. Dafür musste der HTML-Code für das Navigationsmenü aus allen HTML-Seiten (Siehe Abb.15) in eine PHP-Datei ausgelagert werden. Diese Datei enthält nur ein einziges Mal den Navigationsquelltext. Entstehen Änderungen in der Navigation, braucht nur noch diese Datei modifiziert werden und die Änderungen wirken sich sofort auf jede einzelne Seite aus.

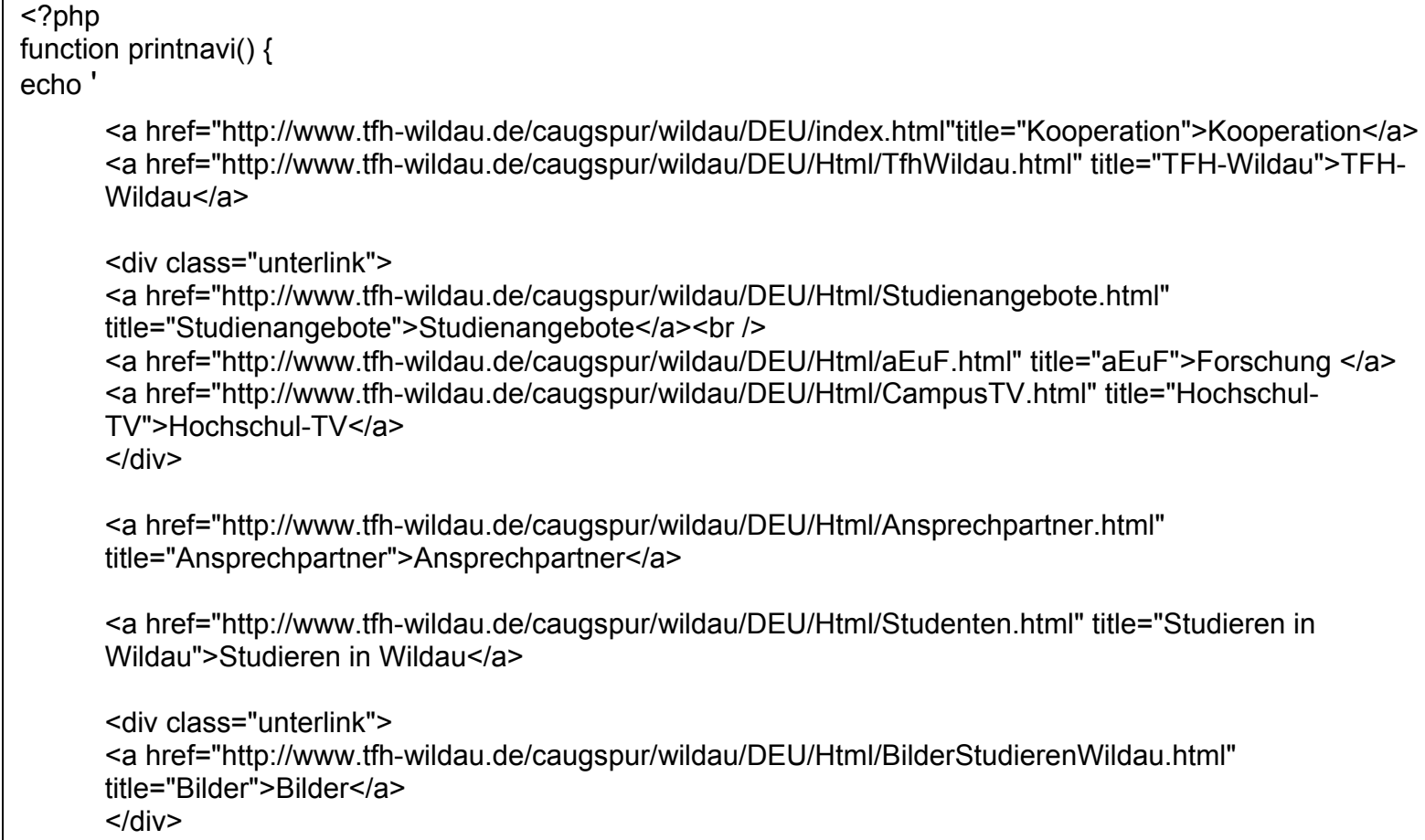

Abbildung 15: Navigationsmenü mit PHP (Ausschnitt)

Diese Funktion hat die Navigationsleiste in jede HTML-Seite integriert. Das bot die Möglichkeit mit wenigen und kurzen Schritten Links an einer zentralen Stelle im Quellcode hinzuzufügen, zu entfernen oder zu verändern.

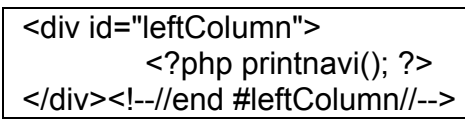

Abbildung 16: Funktionsaufruf für die Navigation

In der spanischen Version wurde entsprechend die Funktion „printnaviesp()“ definiert. 


\subsection{Struktur der Webseite}

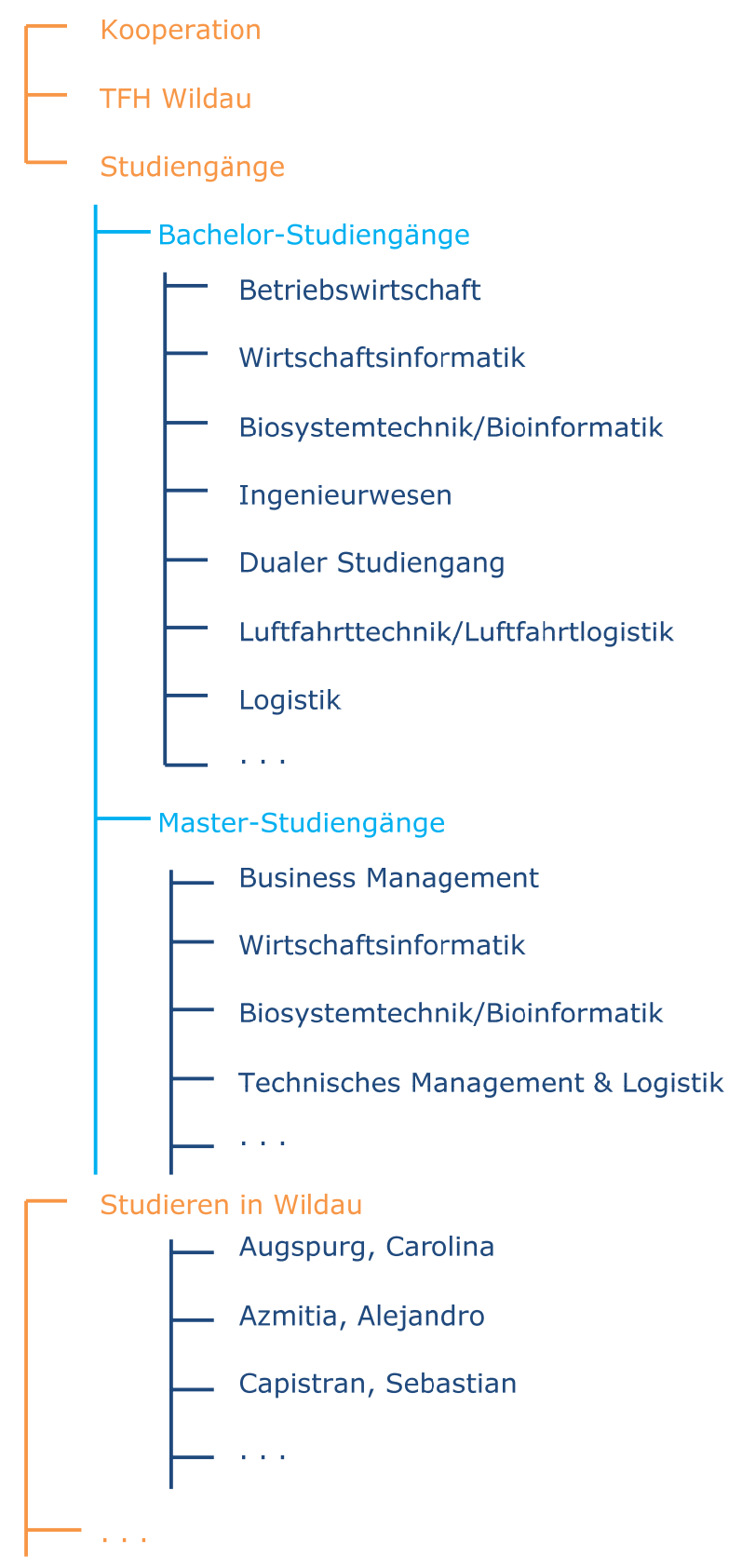

Abbildung 17: Struktur der Webseite (Ausschnitt, vereinfacht)

Die Linien zwischen den Ebenen entsprechen den Verknüpfungen zwischen den Seiten. Die erste Ebene enthält die elf Hauptbereiche der Webseite. Sie bildet die erste Navigationsebene der Webseite (jeweils orange dargestellt).

Einige Bereiche innerhalb der Navigation besitzen eine eigene Navigation (hell blau), diese wiederum enthalten auch Unterpunkte, eine dritte Ebene (dunkel blau). 
Die Hauptbereiche der Webseite sind:

- „Kooperation“

Die Kooperations-Seite ist gleichzeitig die Startseite der Webseite und beschreibt allgemeine Inhalte zur Kooperation, wie z.B. das Kooperationsabkommen und seine Historie.

Diese Seite hat den Zweck, den lateinamerikanischen Schülern einer Deutschen Schule eine grundlegende Idee von den Vorzügen, die die Kooperation der TFH Wildau mit sich bringt, vorzustellen. Die Schüler sollen hier aufgeklärt werden, welche Vorteile ein Studium in Wildau für sie bietet.

Die Informationen hierfür wurden von Prof. Dr. Müller aufbereitet (Siehe Anhang, S. 55).

\section{- „TFH Wildau“}

Mit der TFH-Wildau Seite wird dem Leser die TFH Wildau vorgestellt. An dieser Stelle werden die Tätigkeitsbereiche und Kompetenzen der TFH Wildau beschrieben. Diese Seite berichtet sowohl über die Studierende als auch über die angewandte Forschung und Entwicklung (aFuE) an der TFH Wildau. Dazu zeigen Grafiken die Entwicklung der Studentenzahl und Absolventenzahl der TFH Wildau.

Prof. Dr. László Ungvári (Präsident der TFH Wildau) stellte den Inhalt dieser Seite zur Verfügung (Siehe Anhang, S. 56).

\section{- „Studienangebote“}

Die Studiengänge-Seite besteht aus zwei Unterpunkten (Bachelor- und Masterstudiengänge). In einer tabellarischen Darstellung werden die Studienangebote an der TFH Wildau nach Fachbereichen aufgelistet. Informationen wie Dauer des Studiums, Art des Abschlusses und einen Link zu den Flyern von jedem Studiengang sind in der Tabelle zu finden. Die „Studienangebote-Seite“ enthält ebenfalls einen Link zu den Fachbereichen sowie Studieninformationen aus dem Studienführer 2008/2009.

Umfangreicher Erfahrungsaustausch und Diskussionen führten zu einer optimierten Struktur und Aufbau dieser Seite, so dass große Mengen an Informationen möglichst übersichtlich publiziert werden können. 
Schnelleinstieg für: Studierende | Mitarbeiter | Bewerber | Gäste | Wirtschaft |

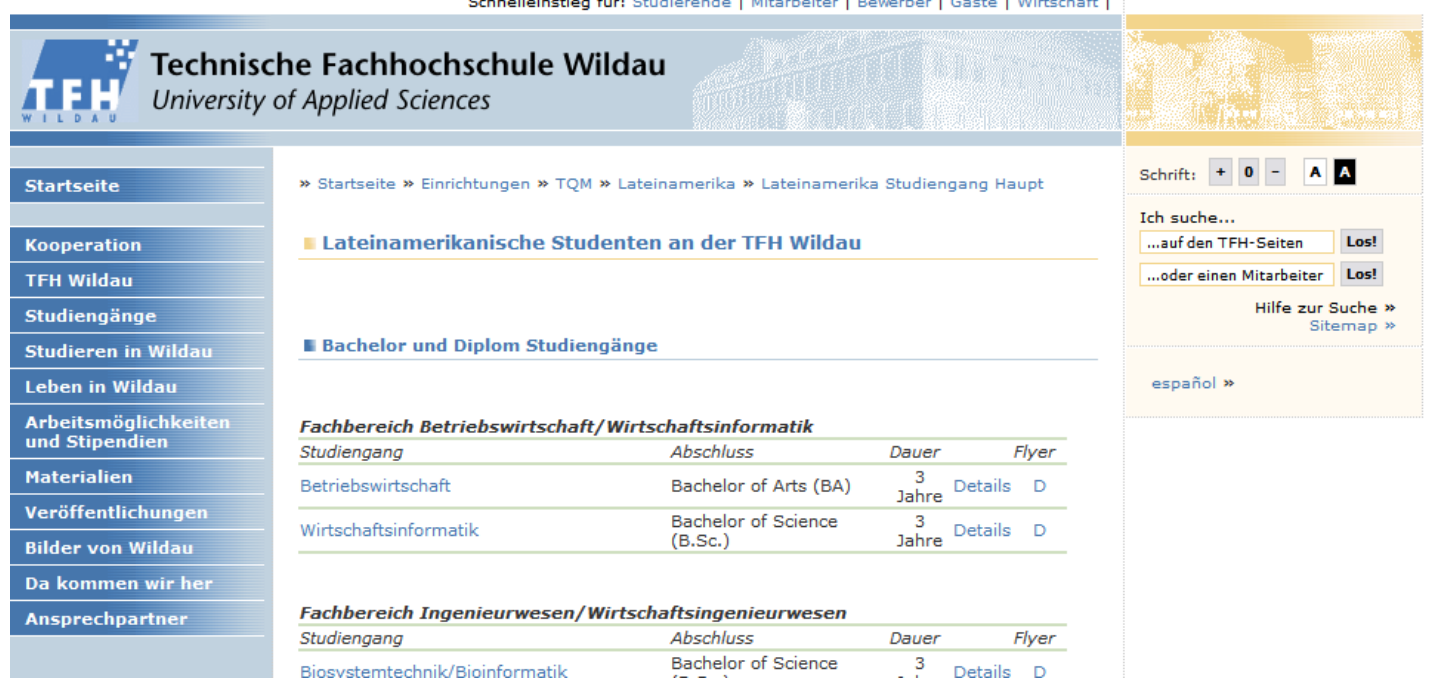

Abbildung 18: Screenshot von "Studiengänge"

Bei der Übersetzung der Seite von Deutsch auf Spanisch wurde besonders Wert darauf gelegt, die Inhalte der Detail-Links bzw. die Seiten mit Informationen zu jedem Studiengang zu übersetzen.

- „Studieren in Wildau“

Die Erstellung dieser Seite erforderte erhebliche Aufwände hinsichtlich der Datenerhebung (Siehe Kap. 3.1. Datenerhebung, Befragungen zu den Profilen).

Hier werden in einer Tabelle alle Namen der lateinamerikanischen Studenten der TFH Wildau aufgelistet. Neben Herkunftsland und Studiengang findet man die Email-Adressen in dieser Tabelle. (Siehe Anhang, S.61-62)

Die Länder-Links führen den Nutzer zu einem Länder-Profil mit Hauptinformationen und Bildern über das jeweilige Land (Siehe Kap. 3.1. Datenerhebung, Umfrage zu den Grunddaten der Heimatländer). 
Schnelleinstieg für: Studierende | Mitarbeiter | Bewerber | Gäste | Wirtschaft |

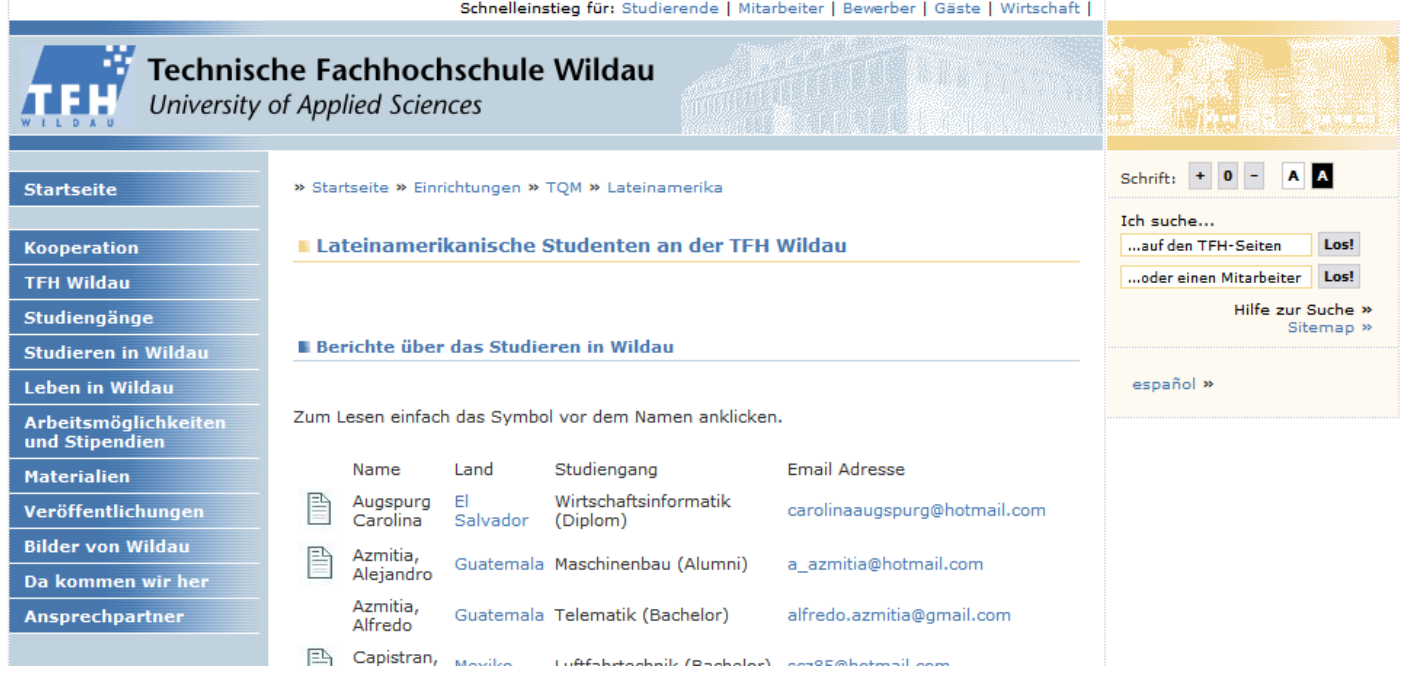

Abbildung 19: Screenshot zu "Studieren in Wildau"

- „Leben in Wildau“

"Leben in Wildau“ ist eine Seite bestehend aus zwei von Studenten geschriebenen Berichten über das Leben in Wildau und das Leben in Berlin.

Diese Berichte tragen dazu bei, dass der Leser (Schüler oder Eltern) sich ein besseres Bild über Wildau bilden kann. Die persönlichen Eindrücke von Studenten spielen dabei eine wichtige Rolle (Siehe Anhang, S. 63).

\section{- „Arbeitsmöglichkeiten und Stipendien“}

Innerhalb der Seite „Arbeitsmöglichkeiten und Stipendien“ (Siehe Anhang, S. 64) geht es um eine explizite Darstellung für ausländische und deutsche Schüler über die Möglichkeiten, die sie in Deutschland haben, als Student Geld zu verdienen. Die Rubriken auf dieser Seite sind: Studentische Hilfskraft, Stipendium, Praktikum, Nebenjob und Fördermöglichkeiten für Deutsche bzw. für Ausländer.

Für die Datenerhebung wurden die lateinamerikanischen Studenten der TFH Wildau befragt und interviewt (Siehe Kap. 3.1. Datenerhebung, Befragungen zu Finanzierungsmöglichkeiten). 
- "Materialien“

An dieser Stelle werden Materialien, Flyer und Poster in Form von pdf-Dateien veröffentlicht. Für jeden Fachbereich findet man den entsprechenden Flyer zu den Studiengängen. Die Poster stehen jeweils auf Deutsch und auf Englisch zur Verfügung. (Siehe Anhang, S. 65).

\section{- „Veröffentlichungen“}

Der Link „Veröffentlichungen“ (Siehe Anhang, S. 66) führt zu Informationen, die für diese Kooperation von Bedeutung sind. Die bis jetzt enthaltenden Informationen sind:

- Jahrbuch Bericht für die Deutsche Schule San Salvador : Bericht für das Jahrbuch der Deutsche Schule San Salvador von salvadorianischen Studenten der TFH Wildau über das Studieren und Leben in Wildau

- Präsentation für die Schüler aus der Deutschen Schule Guatemala: Präsentation über das Studieren und Leben in Wildau von guatemaltekischen Studenten der TFH Wildau

- Bilder vom Besuch von Thomas Castella : Von Thomas Castella (Lehrer an der Deutschen Schule Guatemala) erstellte Bilder zu seinem Besuch in Deutschland

- Videopräsentationen der TFH Wildau: Link zu den Videopräsentationen der TFH Wildau auf verschiedene Sprachen

- „Bilder von Wildau“

Diese Seite (Siehe Anhang, S. 69-70) besteht aus einer Reihe von Unterkategorien mit Bildern über: Wildau und Umgebung, Wohnheime in Wildau, Partys in Wildau und Berlin, Sehenswürdigkeiten in Berlin, Campus und Labore. Hier können die Besucher der Webseite optische Eindrücke von Wildau sammeln (Siehe Kap. 3.1. Datenerhebung, Umfrage den Bildern).

- „Da kommen wir her"

Hauptsächlich die deutschen Interessenten lateinamerikanischer Länder werden mit diesem Link angesprochen (Siehe Anhang, S. 73-74). In einer Liste mit Links zu den Profilen von 
den Heimatländern der lateinamerikanischen Studenten stellen sich Chile, Costa Rica, El Salvador, Guatemala, Kolumbien, Mexiko und Venezuela vor.

Jedes Länderprofil enthält folgende Informationen: Allgemeine Informationen zu dem Land, Bilder so wie eine Auswahl weiterer Informationsquellen (Siehe Kap. 3.1. Datenerhebung, Umfrage zu den Grunddaten der Heimatländer).

Die Länderprofile sollen mit verschiedenen Bildern aus den entsprechenden Heimatländern vervollständigt werden (Siehe Kap. 3.1. Datenerhebung, Umfrage zu Bildern).

Schnelleinstieg für: Studierende | Mitarbeiter | Bewerber | Gäste | Wirtschaft |

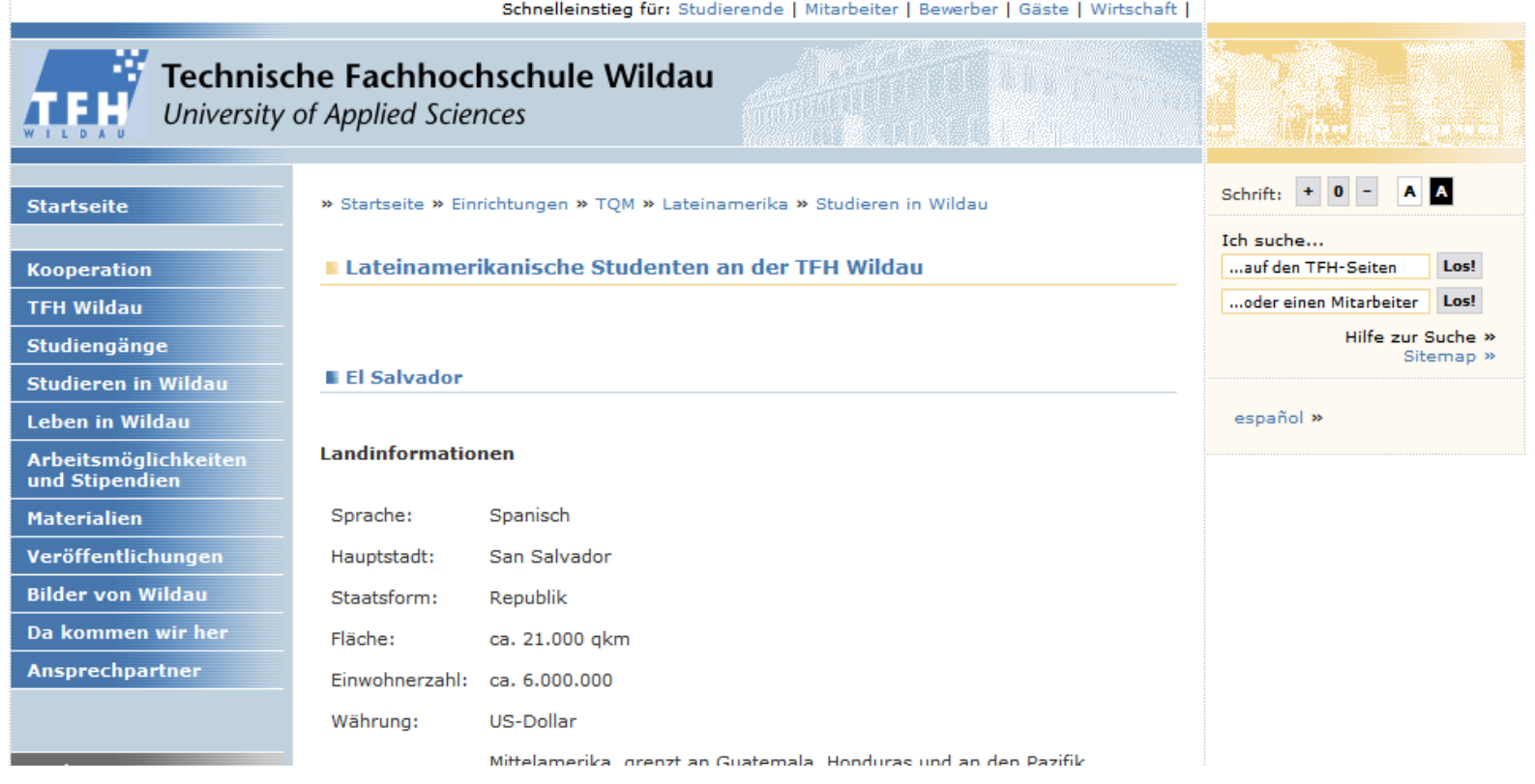

Abbildung 20: Screenshot von "El Salvador"

- „Ansprechpartner“

An dieser Stelle werden die Ansprechpartner der Kooperation und ihre Email-Adressen aufgelistet (Siehe Anhang, S. 75). 


\section{Integration in das RedDot CMS der TFH Wildau}

Nach einer Stärken-Schwächen-Analyse (Siehe Kap. 2.3.1. Vor- und Nachteile des herkömmlichen Webpublishing) des ersten Prototyps mittels HTML, CMS und PHP, wurde entschieden die Webseite in das RedDot CMS der TFH zu integrieren. Diese ist unter: http://www.tfh-wildau.de/lateinamerika/3857.htm zu erreichen.

In dem folgenden Kapitel werden Nutzen und Grenzen, sowie die Benutzeroberfläche des RedDot CMS behandelt. Anschließend erfolgt eine Beschreibung der Einführung der Webseite in das RedDot CMS.

\subsection{RedDot}

\section{$\underline{\text { Über RedDot }}$}

Das „RedDot CMS“ ist ein Content Management und Publishing System. Diese kommerzielle Content Management Lösung koordiniert die Handlungen der Nutzer. „Sie automatisiert die Veröffentlichung der Dokumente, so dass stets aktuelle Online-Publikationen entstehen.“ ${ }^{21}$

„RedDot CMS“ bietet folgenden Nutzen:

- Trennung von Content und Layout

- Strikte Einhaltung eines vorgegebenen Designs (Corporate Identity)

- Dezentrale Pflege von Inhalten

- Effektive Produktion ohne HTML-Kenntnisse

- Permanente Vorschau

- Automatische Linkkonsistenz

- Webbasierter Zugriff auf eine Asset-Ablage

- Integrierte Bildbearbeitungsfunktion

- Definierte Arbeitsabläufe über Workflow (Berechtigungen, Qualitätssicherung) 
Das „RedDot CMS“ wird von einem Content Manager per Webbrowser gepflegt und administriert. Jeder Autor erhält einen eigenen Benutzer-Account mit dem er genau die inm zugewiesene Seiten bearbeiten kann.

Im Gegensatz zu anderen CMS-Systemen findet bei RedDot eine statische Speicherung der Webseiten statt. Diese Methode führt zu einem kürzeren Antwortzeitverhalten und benötigt wesentlich weniger Hardwareressourcen.

Für die Arbeit im „RedDot CMS“ benötigte man trotz Programmierkenntnisse an einigen Stellen technische Unterstützung. Das Programm „RedDot CMS“ und dessen Funktionen bzw. dessen Bedienung haben an bestimmten Stellen technische Fragen aufgeworfen. Für eine reibungslose Einbindung bzw. Aktualisierung von Seiten im „RedDot CMS“ ist daher eine ausführliche Mitarbeiterschulung zu empfehlen.

In dem „RedDot CMS“ lassen sich weitere allgemeingültige Nachteile eines CMS finden, wie z.B: die unflexiblen Gestaltungsmöglichkeiten bei der Inhaltsdarstellung, was einerseits gewollt ist, aber was anderseits die Kreativität des Autors beschränkt.

Besonders nachteilig ist, dass eine hundertprozentige Unterstützung aller verfügbaren Funktionen nur im Internet Explorer gewährleistet wird.

\section{Benutzeroberfläche}

Die Benutzeroberfläche der „RedDot CMS“ Version 7.1 ist in drei Bereiche aufgeteilt:

- Mittlerer Bereich

Im mittleren Bereich der Seite, dem Arbeitsbereich, werden die Inhalte der Seite mit Hilfe der RedDot-Symbole bearbeitet.

- Werkzeugleiste oben

Diese Werkzeugleiste befindet sich oben und ist nur im Seiten-Bearbeitungsmodus zu erreichen. Die freigegebenen Funktionen in dieser Leiste sind schwarz markiert. Gesperrte bzw. deaktivierte Funktionen sind grau hinterlegt.

\begin{tabular}{|l|l|l|l|l|l|}
\hline \multicolumn{2}{|c|}{ Sie bearbeiten die Seite 'Lateinamerika Kooperation' mit Seiten-ID 3858} \\
\hline - Eigenschaften & - Aktionen & - Verlinkung & - Schlagworte & - Bemerkungen & - Seiteninformationen \\
\hline
\end{tabular}

Mit den freigegebenen Optionen, werden Funktionen wie „Seite publizieren“ oder „Seite löschen" sowie verschiedene Seiteninformationen (u.a. eine Auskunft über die Überschrift und die ID der Seite) zur Verfügung gestellt. 
- Werkzeugleiste links

Die Symbolleiste auf der linken Seite enthält folgende Funktionen:

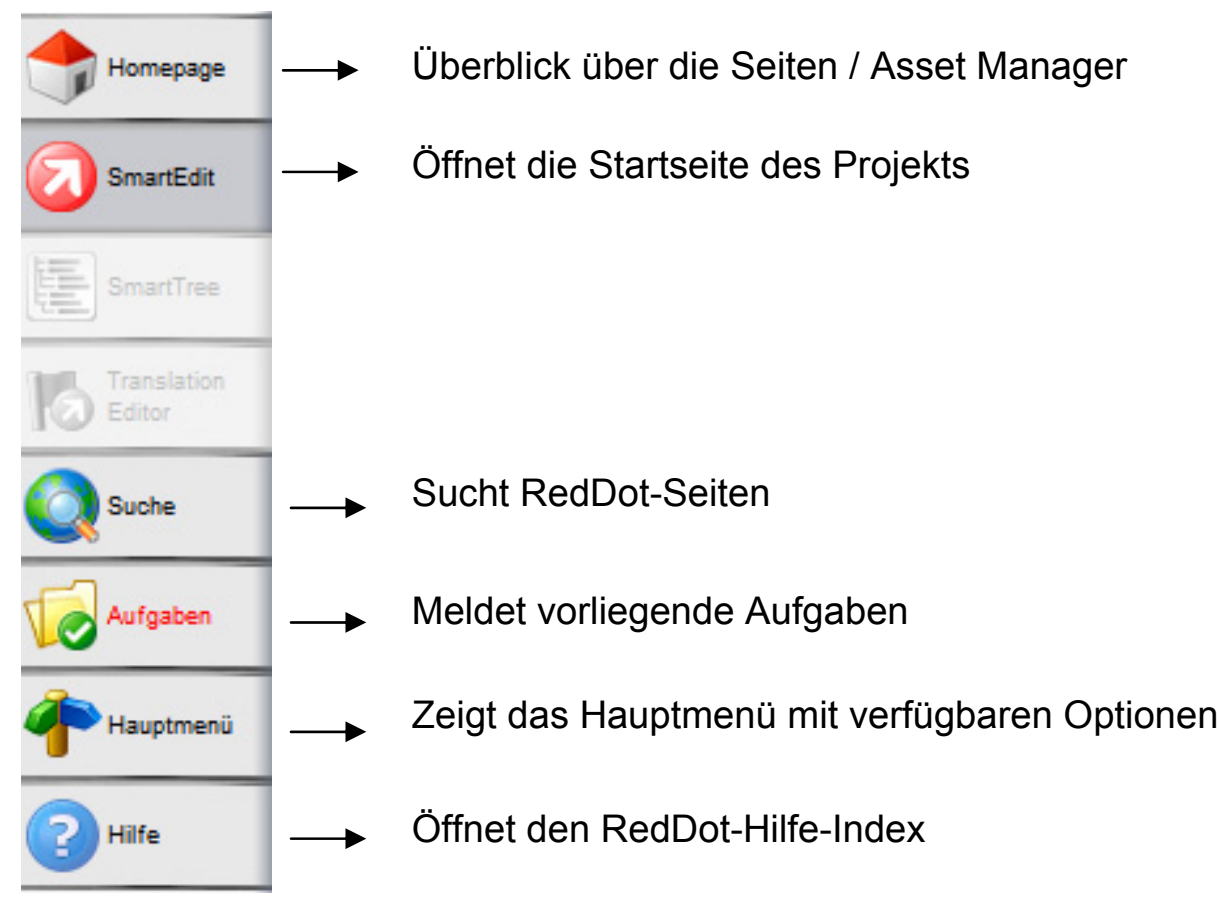

Klickt man auf den Homepage-Knopf in der linken Werkzeugleiste erhält man eine Übersicht über die von dem Benutzer erstellten Seiten, die noch nicht freigegeben wurden, sowie den Zugriff auf den „Asset Manager“.

„Der Asset Manager ist ein Medienverwaltungsordner, dessen Dateien - ohne dass sie wie bisher mit einer Seite verknüpft sind - im Internet publiziert werden (z.B. als Verweis in einer Email)."22

Mit dem Asset-Manager können alle hochgeladenen pdf-Dokumente und Bilder auf dem Server verwaltet werden. Der Asset-Manager besitzt u.a. eine Funktion zur Bildbearbeitung, welche besonders nützlich ist, um Bilder in ihrer Größe zu anzupassen.

[22] R. Tinius (2004) 


\subsection{Einführung der Webseite in das RedDot CMS}

Mit der Überführung des HTML - Prototyps in das RedDot CMS sollten die entstandenen Schwachstellen behoben und die Lateinamerika-Webseite vollständig in den Internetauftritt der TFH Wildau integriert werden.

Mir wurde eine Benutzer-Rolle sowie ein Benutzername und Passwort für die notwendige Anmeldung an das System zugeteilt, um mit dem RedDot CMS arbeiten zu können. Nach einer erfolgreichen Anmeldung, gelangt man auf die Startseite von dem mir zugeordneten neuen Web-Projekt.

Anhand der Seite „Kooperation“ wird nun beispielhaft die Erstellung einer Seite im RedDot CMS erläutert.

\section{Navigation und Erstellung Neuer Seiten}

Zunächst wird dafür in der Navigationsleiste unter dem Punkt „Navi bearbeiten“ ein neuer Link erstellt. Dort findet man eine große Auswahl an Funktionen, die für die Bearbeitung der Navigations-Links nützlich sind.

1. Ein neuer Linkname wird festgelegt (z.B: Kooperation).

2. Klick auf „Seite erstellen und verknüpfen“

Es öffnet sich ein neues Fenster mit einer großen Auswahl an Templates. Für die Erstellung der Webseite wurde ausschließlich mit dem Standard-Template:

„Neu_Leere_Seite_mit_Ueberschrift_und_Textbox“ gearbeitet. Eine identifizierende Kennung bzw. ein Name für die neu erstellte Seite ist erforderlich, um später die Seite im System wiederfinden zu können.

3. Klick auf „Target-Container zuweisen“

Jeder Hauptbereich einer Webseite besitzt im RedDot CMS seinen eigenen TargetContainer. Er stellt das komplette Rahmen-Design (wie Navigation und Überschriften) zur Verfügung. Diese Eigenschaften sind für jeden Bereich unterschiedlich.

Für dieses Projekt wurden zwei verschiedene Target-Container benutzt. Der TargetContainer „Lateinamerikanische Studenten an der TFH Wildau“ für die deutsche Version der 
Seite und „Estudiantes latinoamericanos en la Universidad de Wildau“ für die spanische Version der Seite.

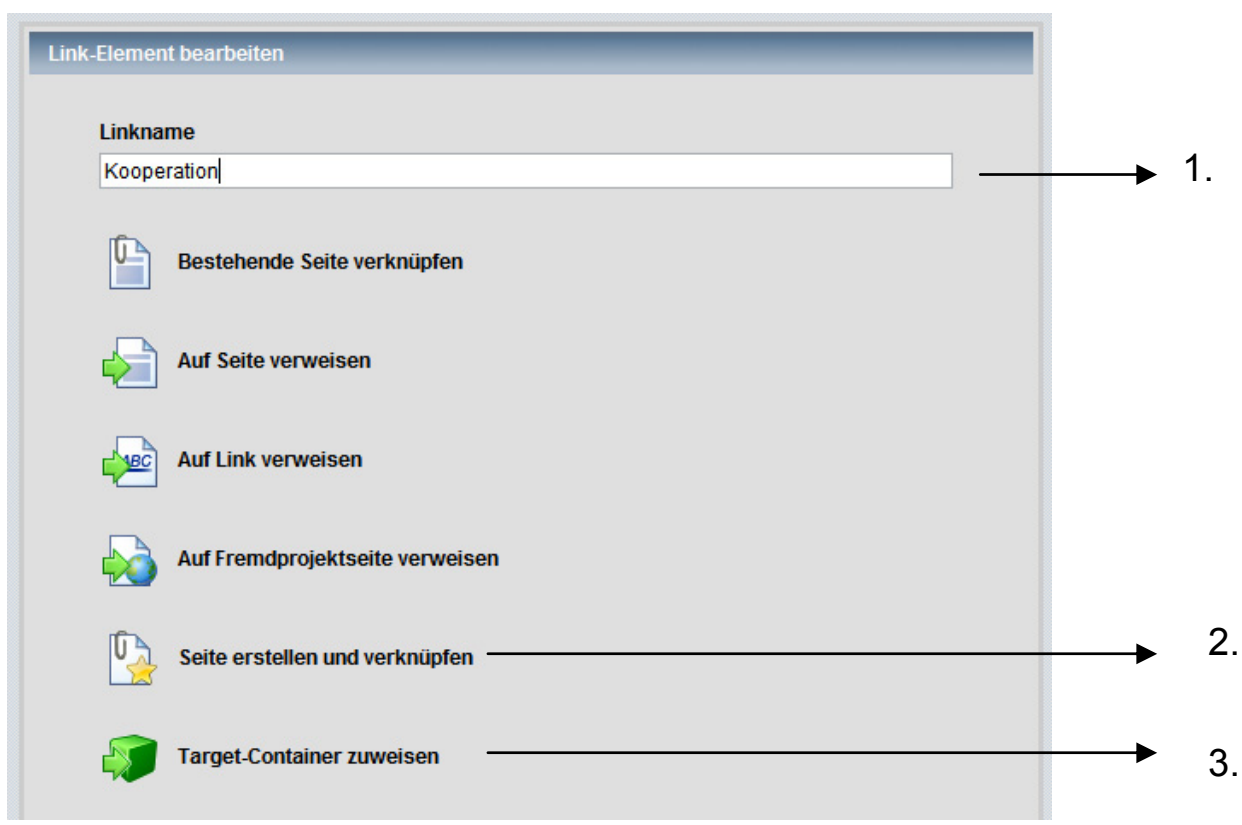

Sind diese drei Schritte erfolgreich durchgeführt, kann man nun innerhalb dieser Seite Inhalte bearbeiten, neuanlegen oder aktualisieren.

\section{Seitenbearbeitung}

Für die Seitenbearbeitung muss der fertiggestellte Link betätigt werden. Es öffnet sich ein Fenster mit einer Auswahl an Optionen für diese Seite.

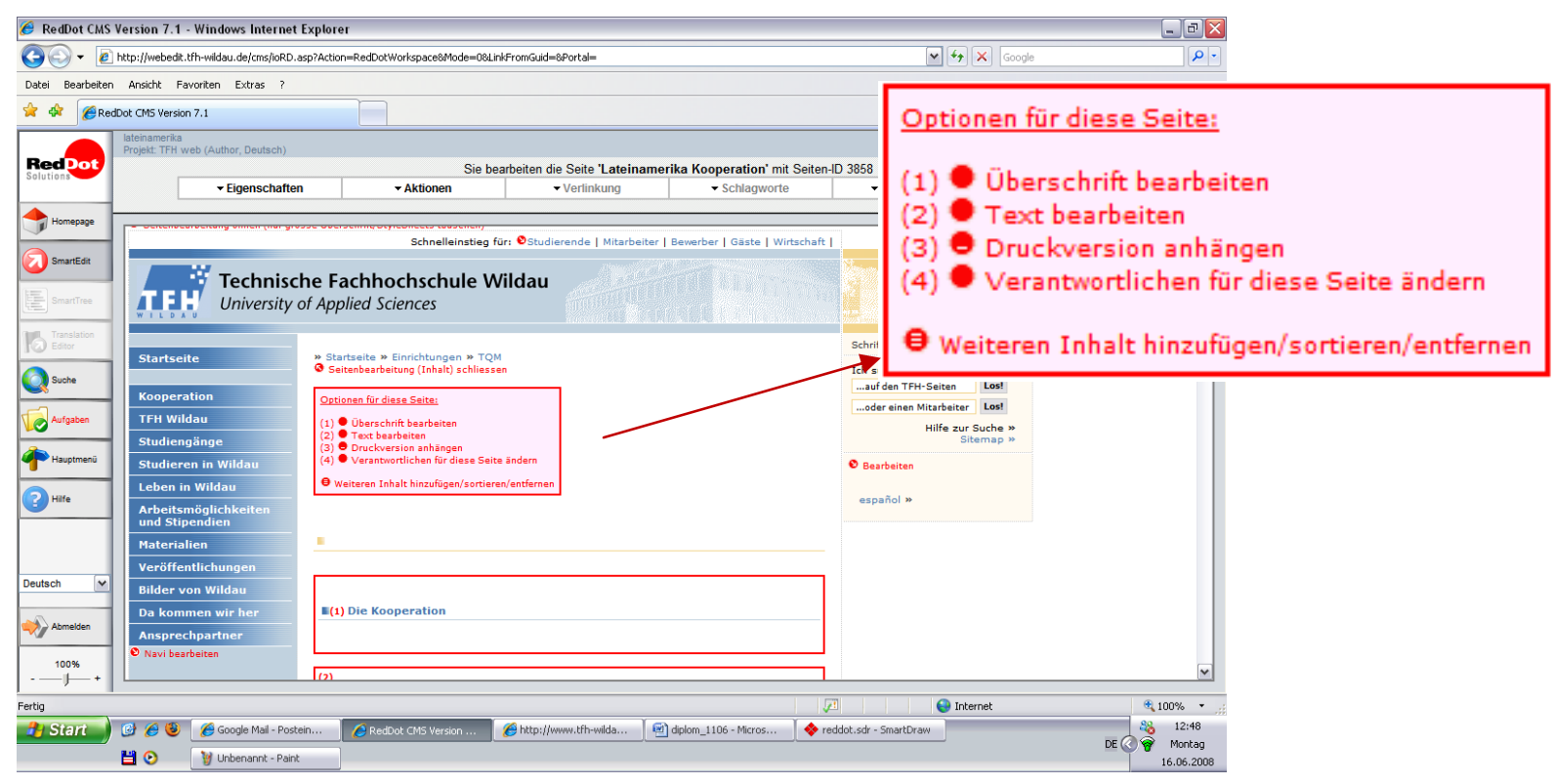

Mit der ersten Option wird ein Titel für die Seite (1) festgelegt (Die Kooperation). 
Mit einem Klick auf den RedDot Punkt „Text bearbeiten“ (2) gelangt man zu einem Texteditor für die Textbearbeitung. Die reinen Textinformationen können hierfür aus den HTML-Seiten des Prototyps übernommen werden. Innerhalb des Texteditors ist eine Leiste mit verschiedenen Funktionen sowie ein Feld für die Textbearbeitung zu finden.

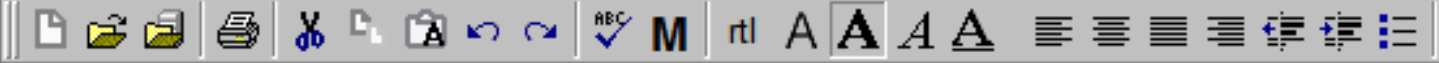

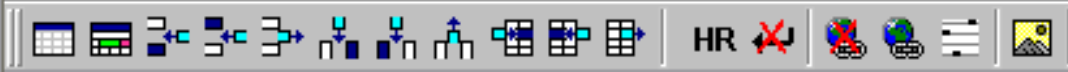

Die Darstellung der möglichen Textbearbeitungsfunktionen ist besonders benutzerfreundlich. Einige Funktionen, die für die Entwicklung der Lateinamerika-Webseite nützlich waren, sollen an dieser Stelle erwähnt werden:

\section{- Tabellen erstellen}

Tabellen werden mit dem Symbol $\square$ erstellt und mit dem Symbol $\sqrt{\mathbf{m}}$ bearbeitet.

Es können sowohl die Anzahl der Spalten und Zeilen innerhalb der Tabelle als auch die Breite und Farbformatierungen festgelegt werden.

Zusätzlich kann man nachträglich Zeilen, Spalten oder Zellen einfügen bzw. löschen.

\section{- Links erstellen}

Für die Erstellung eines Links muss zunächst der Text oder das Bild, welcher/-s später einen Link enthalten soll, markiert werden. Dann wird das Symbol geklickt. Dabei öffnet sich eine Maske mit verschiedenen Optionen.

Auf der Lateinamerika-Webseite findet man Verlinkungen zu externen Seiten (z.B. Webpages der lateinamerikanischen Deutschen Schulen innerhalb der Kooperation) sowie zu bereits in RedDot erstellten Webseiten.

Für die Verlinkung auf externe Seiten gibt man im „href“-Feld die URL-Adresse der Internetseite, auf die der Link verweisen soll, ein. 


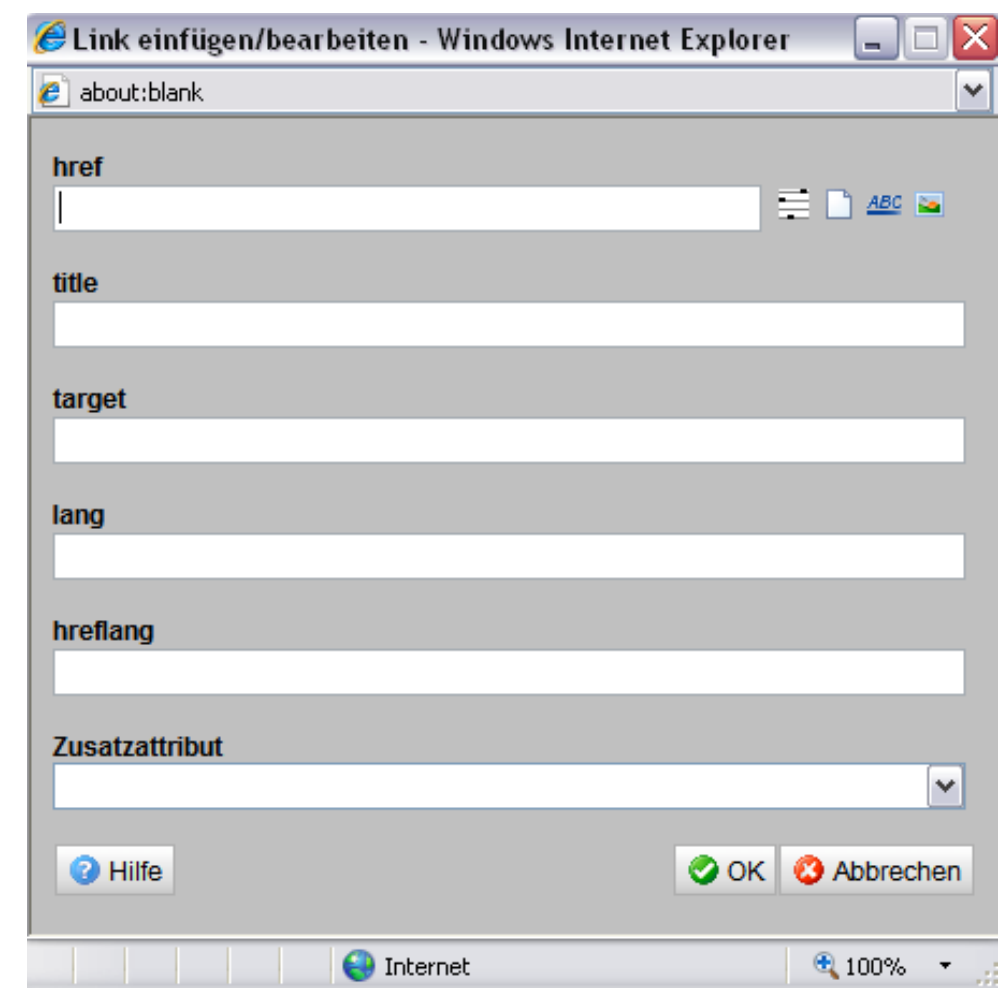

Ähnlich wie in HTML können auch hier Target-Eigenschaften für die Links festgelegt werden.

Für die Einbindung von einer bereits erstellten Seite in RedDot muss man das $\square$ Symbol benutzen. Es öffnet sich eine Suchmaske, in der man verschiedene Suchkriterien angeben kann, um die gewünschte Seite zu finden. Die gefundene Seite erscheint im „href“ Feld und kann analog wie eine externe Seite verlinkt bzw. eingebunden werden.

\section{- Bild einfügen}

Für die Speicherung der Bilder, Grafiken und pdf-Dateien wurde serverseitig der Ordner „Dokumente Lateinamerika“ zur Verfügung gestellt.

Bilder können mit einem Klick auf das Symbol eingefügt werden. Es gibt die Wahlmöglichkeit, das Bild von einer Festplatte hochzuladen oder ein bereits in RedDot CMS vorhandenes Bild zu benutzen.

Beim Hochladen von lokalen Bildern muss mit der Suchfunktion der richtige Dateipfad angegeben werden. Die Auswahl des richtigen Zielordners (Dokumente Lateinamerika) im RedDot CMS ist erforderlich. 
Möchte man auf dem Server bereits existierende Dateien einbinden, muss man im entsprechenden Ordner danach suchen.

Das Hochladen von neuen Bildern bedeutete einen hohen Zeitaufwand, da die Bilder nur einzeln hochgeladen oder bearbeitet werden können.

\section{Erstellen von neuen RedDot Seiten (außerhalb der Navigation)}

Das Erstellen von neuen RedDot Seiten konnte, im Vergleich zu den meisten Funktionen von RedDot, nicht intuitiv erfolgen. Nach einer Einweisung seitens der Techniker des Hochschulrechenzentrums an der TFH Wildau konnte diese Herausforderung jedoch schnell gemeistert werden.

Für den Aufbau der Webseite war eine große Anzahl dieses Seitentyps (neue Seite außerhalb der Navigation), der in RedDot unter dem Namen „unsichtbare Links“ bekannt ist, erforderlich.

Anfangs wird für die Erstellung solcher Seiten der Punkt „Weiteren Inhalt hinzufügen/entfernen“ ausgewählt. Diese Funktion befindet sich im SeitenverarbeitungsMenü. Dabei öffnet sich eine Maske mit verschiedenen Funktionen, aus der die Funktion „neue Seite erstellen und verknüpfen“ ausgewählt wird. Hier besteht das Angebot, ähnlich wie bei der Erstellung von neuen Seiten für die Navigation, ein Template zu wählen. Anders als die bereits kennengelernte Variante, wird einer neuen Seite das Template „ContainerInhalte $\rightarrow$ Dynamischer_Link_nicht_sichtbar“ zugewiesen. Der Target-Container bleibt in der deutschen Version „Lateinamerikanische Studenten an der TFH Wildau“ und in der spanischen Version „Estudiantes latinoamericanos en la Universidad de Wildau“.

Wird eine neue Seite auf die Weise angelegt, kann sie mit einem Link auf bereits erstellten Seiten in das Webprojekt hinzugefügt und verknüpft werden.

Diese „unsichtbaren Links“ werden am unteren Ende der Seitenansicht dargestellt und bieten alle bekannten Seitenbearbeitungsfunktionen. 


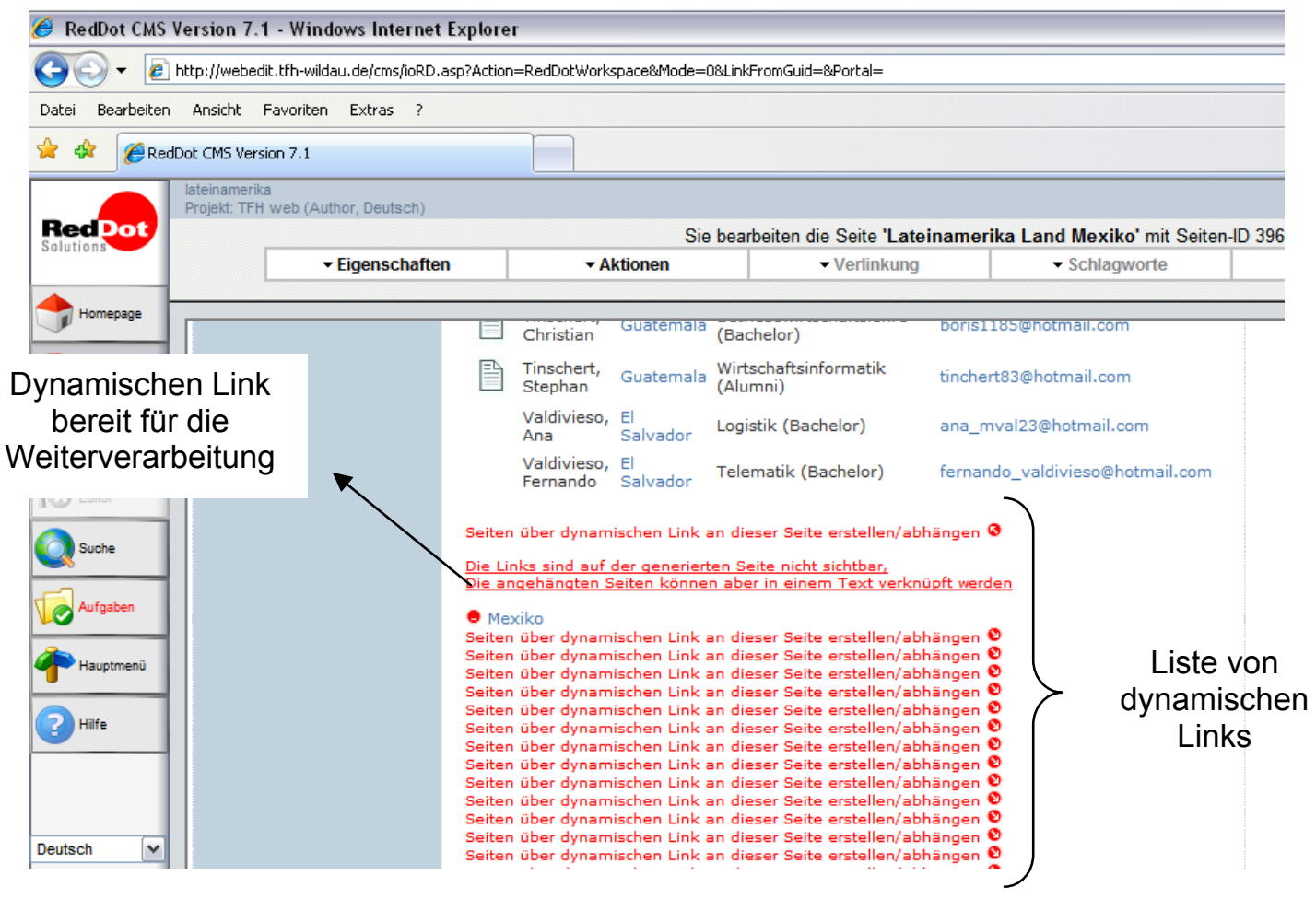

\section{Spanische Version}

Erst nach Fertigstellung der deutschen Version war die spanische Version zu realisieren.

Dafür wurde eine 1:1 Kopie der deutschen Version der Webseite erstellt. Diese Kopie der Webseite ist unter dem Link „español“ auf der rechten Seite der Webseite (orangenen Balken) zu erreichen.

In diesem Duplikat waren lediglich die inhaltlichen Aspekte zu ersetzen, die aus dem Prototyp stammen.

Schnelleinstieg für:

\begin{tabular}{|l|l|}
\hline Startseite \\
\hline Kooperation \\
\hline TFH Wildau \\
\hline Studiengänge \\
\hline Studieren in Wildau \\
\hline Leben in Wildau \\
\hline Arheitsmönlichkeiten \\
\hline
\end{tabular}
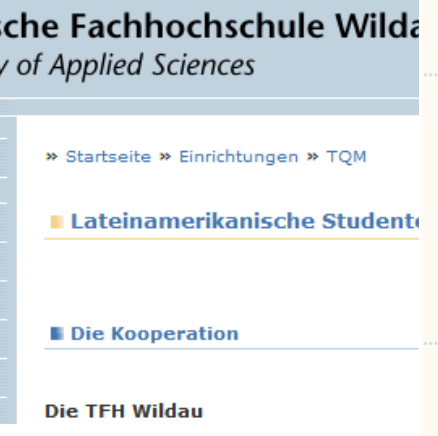

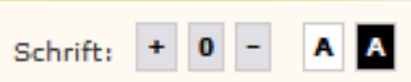

Ich suche...

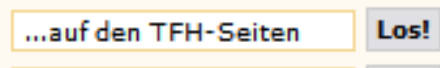

...oder einen Mitarbeiter Los!

Hilfe zur Suche $»$

Sitemap $»$

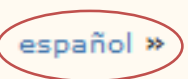




\section{Erweiterbarkeit der Webseite}

Die Webseite für die Lateinamerika - Kooperation an der TFH Wildau wird zukünftig kontinuierlich weiterentwickelt und ausgebaut. Das folgende Kapitel stellt mögliche Erweiterungen für die Webseite vor.

\subsection{Mehrsprachigkeit}

Es besteht die Anforderung, diese Webseite in verschiedene Sprachen zu übersetzen, um so alle Zielgruppen erreichen zu können.

Zurzeit werden die Inhalte der Webseite auf deutsch und auf spanisch im Internet bereitgestellt.

Eine englische Version der Webseite ist bereits in der Planung. Mit dieser Option könnten Studenten aus anderen, nicht lateinamerikanischen Ländern die Leistungen und Vorzüge der TFH Wildau kennenlernen (z.B. Studenten aus Russland, Ungarn oder Polen).

\subsection{Webportal}

Eine weitere Idee für die Erweiterung der Webseite ist die Einführung eines Webportals. Es könnten Web 2.0 Funktionen für eine Social Networking Community integriert werden.

Die Grundidee von Web 2.0 ist, dass der Benutzer selbst Inhalte im Internet erstellen und bearbeiten kann und somit gleichzeitig Informationen konsumiert und produziert.

„Unter Social Network versteht man heute Plattformen bzw. Webseiten (MySpace, StudiVZ) innerhalb des Web 2.0 Kosmos, die ein „Freundenetzwerk“ als virtuelles Interaktionsgeflecht darstellen. ${ }^{“ 23}$

Dabei kann ein Benutzer i.d.R. ein persönliches Profil von sich selbst erstellen, um sich dort vorzustellen und mit anderen Menschen im Netzwerk Kontakt aufzunehmen. Die Kontaktaufnahme funktioniert meist durch das Senden von Freundschaftsanfragen. Mit einer Reminder-Funktion besteht die Möglichkeit die Nutzer an kommenden Events oder Geburtstage zu erinnern.

[23] J. Leibiger, J. Fürst, S. Redlich, B. Schröder, M. Bruno, D. Dank (2006) 
Oft existieren zusätzliche Funktionen, um Bilder- und Videodateien in die Profile einzubinden und so persönliche Lieblingsmusik, Filme oder Fotos im Freundeskreis vorzustellen. Diese lassen sich von den Freunden jeweils bewerten oder kommentieren, was letztendlich auch eine Form der Kontaktaufnahme darstellt.

Die Einbindung solcher Plattformfunktionen in die Webseite der Lateinamerika Kooperation an der TFH Wildau könnte den Kommunikations- und Informationsaustausch zwischen den Nutzern wesentlich erweitern.

Social Networking-Dienste können auch den Aktualisierungsprozess der Webseite automatisieren, da viele Informationen selbst von den Nutzern erstellt und aktualisiert werden.

Nach einer ersten Anmeldung im Portal könnte der Benutzer aufgefordert werden, ein Profil über sich zu erstellen und u.a. Informationen über seinen Studiengang oder seine Erfahrungen in Wildau vorzustellen. Der Aufbau sollte den Profilen der „Studieren in Wildau"Seite entsprechen, mit dem Unterschied, dass die Informationen selbst vom Benutzer erstellt, erfasst und publiziert werden. Die Aktualisierung der Bilder würde gleichermaßen ablaufen, da jeder Benutzer in der Lage wäre, eigene Bilder hochzuladen.

Ein Schüler aus einer Deutschen Schule im Ausland könnte sich im Portal anmelden und nach Studenten suchen, die z.B. Wirtschaftsinformatik studieren. Es besteht dann die Möglichkeit einer Kontaktaufnahme.

\subsection{Weblog}

„Der Begriff Weblog ist ein zusammengesetztes Wort aus „Web“ und „Logbuch“ - das ist im Grunde eine öffentlich zugängliche Webseite, auf der mehr oder minder regelmäßig neue Beiträge veröffentlicht werden. “24 Sie bieten dem Nutzer die Möglichkeit Informationen, Gedanken und Erfahrungen auszutauschen.

In einem Blog hätten Schüler aus Deutschen Schulen in Lateinamerika die Möglichkeit Beitrage zu kommentieren bzw. Fragen zu einem bestimmten Thema zu stellen. 


\subsection{Video Conferencing}

Eine andere Erweiterung könnte die Einführung von Video-Conferencing auf der Webseite sein.

Die grundlegende Idee ist, halbjährlich oder jährlich Termine festzulegen, an dem VideoConferencing statt findet. Diese Termine werden mit den Deutschen Schulen in Lateinamerika abgestimmt. Dabei würden sich die Schüler aus den entsprechenden Deutschen Schulen sammeln und eine Präsentation über das Leben und Studieren in Wildau über einen Live-Video-Stream anhören. Fragen könnten ebenfalls beantwortet werden.

Zu den Vorzügen einer Videokonferenz gehört, dass die jährlichen Reisen nach Lateinamerika, zur Präsentation der TFH Wildau, entfallen könnten. Es entstehen so neue wertvolle Kommunikationswege zur Kontaktaufnahme mit Schülern und Eltern in Lateinamerika. Zeitaufwand und Kosten können ohne Verlust des persönlichen Kontaktes reduziert werden.

\subsection{Ersetzen der „Fachbereiche-Seite“ im Internet-Auftritt der TFH Wildau}

Im Laufe der Entwicklung entstand die Idee, die „Studiengänge-Seite" der LateinamerikaWebseite als hochschulweite „Fachbereiche-Seite" innerhalb der TFH Wildau Homepage zu nutzen, da diese für die Kooperations-Webseite übersetzt wurde. Dafür wurden auf die Informationen aus dem Studienführer zugegriffen.

Die „Studiengang-Seite" enthält sowohl einen Link zu den Fachbereichen der TFH Wildau, als auch einen Link zu den Informationen aus dem Studienführer und bietet somit einen Zusammenhang beider Inhalte. Es entsteht, im Vergleich zu der aktuellen Darstellung der Fachbereiche auf der TFH Homepage, eine Zwischenebene. Diese kann zentral verwaltet werden und verfügt über ein einheitliches Layout und Struktur. 


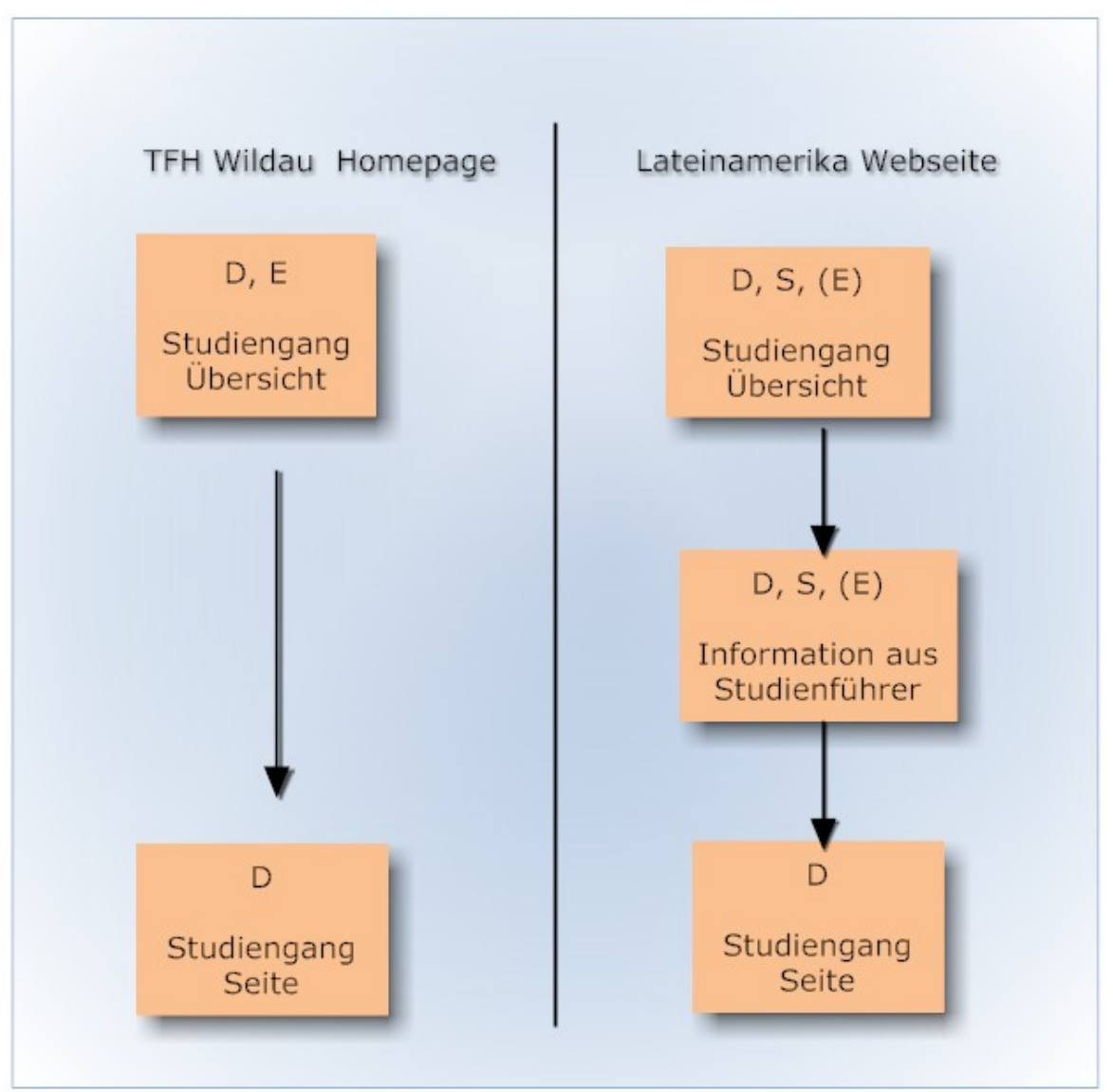

Abbildung 21: Ersetzen der „Fachbereiche-Seite“ im Internet-Auftritt der TFH Wildau

Angebote von Studiengängen der TFH Wildau sind mehrsprachig und einheitlich dargestellt und können in die Homepage der TFH Wildau übernommen werden. 


\section{Zusammenfassung und Ausblick}

Diese Diplomarbeit behandelte das Thema „Konzeption und Entwicklung einer Webseite für die Lateinamerika-Kooperation an der TFH Wildau“ mit dem Ziel den Kommunikations- und Informationsaustausch der lateinamerikanischen Schüler und Studenten der TFH Wildau zu optimieren und eine Basis für das Kooperationsmarketing der TFH Wildau zu schaffen.

Zu Beginn der vorliegenden Arbeit wurde gezeigt, welche technischen Grundlagen für eine Umsetzung der Webseite nötig waren. Mit dem verbesserten Verständnis für die Begrifflichkeiten HTML, CSS und PHP ist anschließend eine Begriffserklärung, sowie die Eigenschaften und Komponenten von WCMS erläutert worden.

Nach dem Datenerhebungsprozess für den Inhalt der Webseite wurde ein Prototyp mit HTML, CSS und PHP entworfen. Durch die kontinuierliche Steigerung der inhaltlichen und strukturellen Anforderungen an die Webseite entstand ein enormer Pflege- und Aktualisierungsaufwand bei der statischen Implementierung, da bei jeder Veränderung eine große Anzahl an HTML-Seiten verändert werden musste.

Die Integration der Webseite in das RedDot CMS zeigte den direkten Vergleich zwischen der Entwicklung der Webseite mit HTML und einem Content Management System. Dabei wurden Vor- und Nachteile beider Vorgehensweisen betrachtet. Die Ergebnisse dieser Analyse zeigen, dass die Entwicklung der Webseite im RedDot CMS die Struktur und Ordnung der Inhalte optimiert hat. Die Veränderungsaufwand wurde reduziert und die Corporate Identity der TFH Wildau übernommen.

Das Ziel, eine Kommunikations- und Informationsplattform für die Lateinamerika-Kooperation zu entwickeln, wurde erreicht. Bereits heute sind Verlinkungen der Webseite in InternetAuftritten der Deutschen Schulen mit einem positiven Feedback zu verzeichnen.

Das Projekt „Lateinamerikanische Studenten an der TFH Wildau" wird fortgeführt und ausgebaut. Dafür wurden im Kapitel „Erweiterbarkeit der Webseite" bereits einige Ideen für die Weiterentwicklung der Webseite beschrieben.

Diese Webseite wird im Laufe ihres Einsatzes und der damit verbundenen Fortentwicklung weiter an Bedeutung gewinnen. Davon bin ich überzeugt. 


\section{Abkürzungsverzeichnis}

CSS

Cascading Style Sheets

CMS

Content Management System

DB

Datenbank

HTML

Hypertext Markup Language

ODBC

Open Data Base Connectivity

PHP

Hypertext Preprocessor

SQL

Structured Query Language

TFH

Technische Fachhochschule

XML

Extended Markup Language

WCMS

Web Content Management System 


\section{Literaturverzeichnis}

Broda, Stephan (2002)

Marketing Praxis, 2. Auflage, Wiesbaden: Gabler Verlag

Brose, Steffen (2008)

Web Anwendungen Programmieren mit PHP, unter: http://www.tu-

chemnitz.de/urz/kurse/unterlagen/php/php.html, abgerufen am 4.5.2008

Engesser, H.; Claus V, Schwill A. (1993):

Duden Informatik. In:Dudenverlag, Mannheim

Glantsching, Peter (2004):

Diplomarbeit: Innovative Content Management Systeme im Betrachtungsfeld von Java 2

Enterprise Edition, Technische Universität Granz

Hömberg, Kay; Jodin, Dirk; Leppin, Maren (2004)

Methoden der Informations- und Datenerhebung, unter: https://eldorado.uni-

dortmund.de/bitstream/2003/21609/1/04002.pdf, abgerufen am 10.5.2008

It-Lexikon (2008)

unter: http://www.itwissen.info/definition/lexikon/content-Inhalt.html, abgerufen am 19.5.2008

Jablonski, Stefan; Meiler, Christian (2001)

Web-Content-Management-Systeme, unter: http://www.wiwitest.uni-

tuebingen.de/cms/fileadmin/Uploads/Web-CMS.pdf, abgerufen am 21.5.2008

Köhle, H. (2008)

Elektronisches Publizieren (X)HTML und CSS, unter: http://www.math-inf.unigreifswald.de/ koehler/publiz/html2.pdf, abgerufen am 3.5.2008

Krugman, Nils (2001)

HTML, XHTML und CSS, unter: http://www.ra.cs.uni-

tuebingen.de/lehre/ss01/pro_internet_ausarbeitung/proseminar_krugmann_ss2001.pdf, abgerufen am 28.4.2008

Leibiger, Johannes; Fürst, Johannes; Redlich, Sören; Schröder, Björn; Bruno, Matthias; Dank Daniel (2006)

Projektarbeit: Social-Networking Plattformen - Nutzen und Möglichkeiten, unter:

http://www.bernd-schmitz.net/skripte/Social-Networking.pdf, abgerufen am 1.6.2008

PHP: Einleitung - Manual (2008)

unter: http://www.php.net/manual/de/introduction.php, abgerufen am 10.5.2008 
Rothfuss, Gunther; Ried, Chirstian (2001)

Content Management mit XML, Berlin, Heidelberg

Prof. Dr. Rieder H. K.; Dipl.-Volksw. Witter-Rieder (2000)

Konzeptionelle und organisatorische Aspekte der erfolgreichen Internet-Nutzung im Tourismus, unter: http://wirtschaft.fh-trier.de/ri/lit/liechtenstein2000.pdf, abgerufen am 3.05.2008

Tinius, Ron (2004)

Diplomarbeit: Einführung eines Content Management System für den Internetauftritt der Technischen Fachhochschule Wildau, Technische Fachhochschule Wildau

Zschau, Oliver; Traub Dennis; Zahradka, Rik. (2001)

Web Content Management, Websites professionell planen und betreiben, Galileo Press

Wolff, Rochus (2004)

Blogs! Text und Form im Internet, unter: http://www.kritische-

ausgabe.de/hefte/reich/wolff.pdf, abgerufen am 18.6.2008

Zschau, Oliver (2000)

Der Content Life Cycle unter:

www.contentmanager.de/magazine/artikel_5_der_content_life_cycle.html, abgerufen am 28.4.2008

Zschau, Oliver (2000)

Tutorium - Web Content Management Systeme unter: www.uni-duesseldorf.de/dfntag2000/downloads/Zschau/ozs-WCMS.ppt, abgerufen am 10.5.2008 


\section{Abbildungsverzeichnis}

Abbildung 1: Darstellung der Funktionsweise von PHP ....................................................

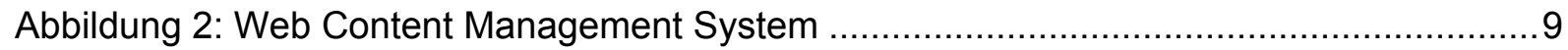

Abbildung 3: Funktionsweise eines Content Management Systems..................................10

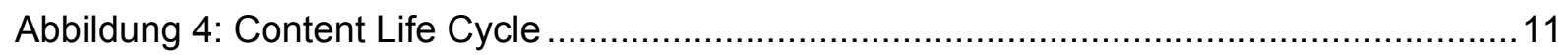

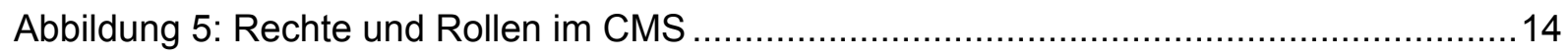

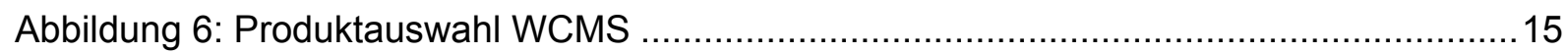

Abbildung 7: Exponentiell wachsender Pflegeaufwand ohne CMS .................................17

Abbildung 8: Herkömmliches Webpublishing ..................................................................... 18

Abbildung 9: Webpublishing mit und ohne Content Management Systeme ....................... 19

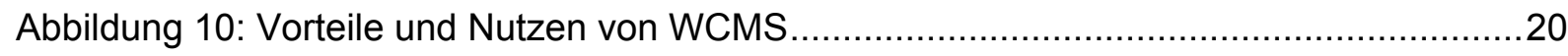

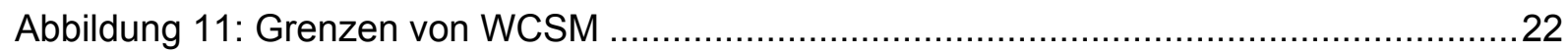

Abbildung 12: Methoden der Informations- und Datenerhebung ....................................24

Abbildung 13: Lateinamerikanische Studenten an der TFH Wildau ....................................26

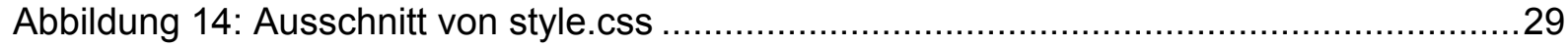

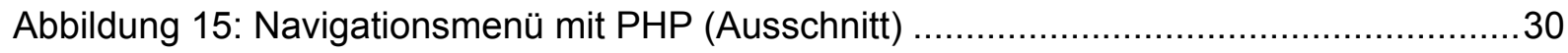

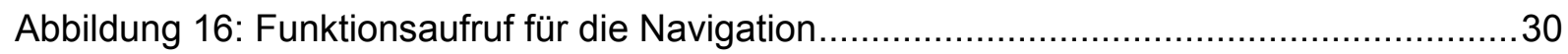

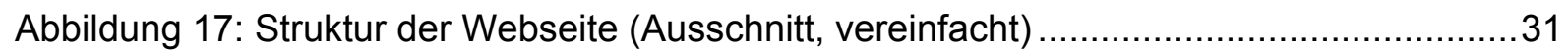

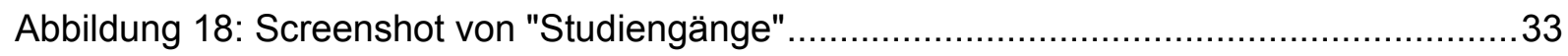

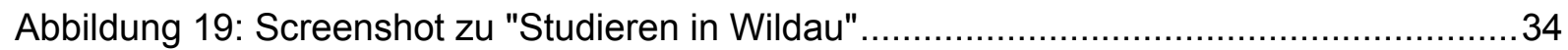

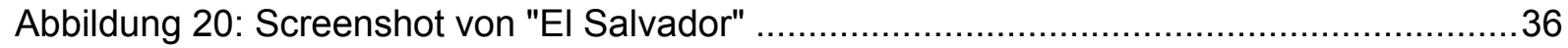

Abbildung 21: Ersetzen der „Fachbereiche-Seite“ im Internet-Auftritt der TFH Wildau..........49 


\section{Anhang}

\section{Lateinamerikanische Studenten an der TFH Wildau $\rightarrow$ Kooperation}

Schnelleinstieg für: Studierende | Mitarbeiter | Bewerber | Gäste | Wirtschaft |

\begin{tabular}{|c|c|}
\hline Startseite & \multirow{2}{*}{$\begin{array}{l}\text { " Startseite " Einrichtungen " TQM } \\
\text { " Lateinamerikanische Studenten an der TFH Wildau }\end{array}$} \\
\hline Kooperation & \\
\hline TFH Wildau & \multirow[b]{3}{*}{ Eie Kooperation } \\
\hline Studiengänge & \\
\hline Studieren in Wildau & \\
\hline Leben in Wildau & \multirow{7}{*}{$\begin{array}{l}\text { Die TFH Wildau } \\
\text { Die TFH Wildau ist mit über } 3500 \text { Studenten die größte Fachhochschule im Land Brandenburg } \\
\text { und liegt am Stadtrand von Berlin. Mit der S-Bahn ist das Berliner Zentrum in } 30 \text { Minuten } \\
\text { erreichbar. Die TFH Wildau ist eine sehr junge und modern ausgestattete Hochschule und } \\
\text { bietet viele vorzügliche technische und wirtschaftswissenschaftliche Studiengänge an. } \\
\text { Gemessen an Mitteleinwerbungen pro Hochschullehrer hat die TFH Wildau eine führende Rolle } \\
\text { unter den Fachhochschulen in Deutschland. Dieses Wissen aus den Forschungsvorhaben fließt } \\
\text { auch sehr schnell in unsere Lehrplane ein. Somit sind diese durch Aktualitat und durch ein } \\
\text { hohes Niveau und starken Praxisbezug geprägt. }\end{array}$} \\
\hline $\begin{array}{l}\text { Arbeitsmöglichkeiten } \\
\text { und Stipendien }\end{array}$ & \\
\hline Materialien & \\
\hline Veröffentlichungen & \\
\hline Bilder von Wildau & \\
\hline Da kommen wir her & \\
\hline Ansprechpartner & \\
\hline \multicolumn{2}{|l|}{ Suche } \\
\hline \multicolumn{2}{|l|}{ Impressum } \\
\hline Sitemap & 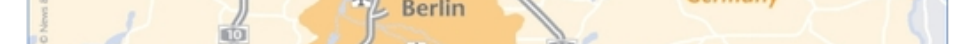 \\
\hline & Al Magdeburg/ \\
\hline
\end{tabular}

Die Kooperationen

Ebenso wichtig wie eine gute fachlich fundierte Ausbildung sind jedoch die Entwicklung und Pflege von internationalen Kontakten zwischen den Studierenden. Wir alle wissen, dass solche frühen Kontakte oft die Basis für spatere erfolgreiche Geschäftsbeziehungen sind. Deshalb versuchen wir gezielt, unseren Anteil lateinamerikanischer Studenten zu erhöhen. Zur Erreichung dieses Zieles unterhalten wir Kooperationen mit:

- der Deutschen Schule in Guatemala,

- der Deutschen Schule in El Salvador.

- der Deutschen Schule in Costa Rica una

- der Deutschen Schule in Cali, Kolumbien.

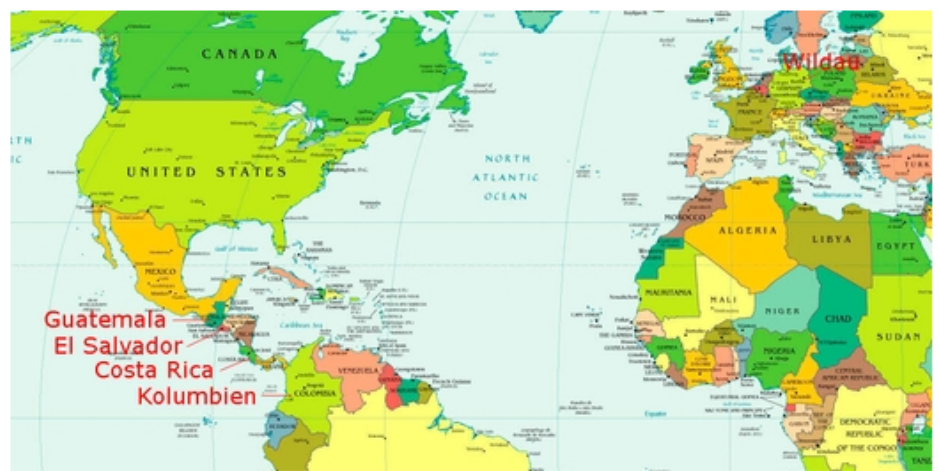




\section{Lateinamerikanische Studenten an der TFH Wildau $\rightarrow$ TFH Wildau}

Schnelleinstieg für: Studierende | Mitarbeiter | Bewerber | Gäste | Wirtschaft |

\section{Technische Fachhochschule Wildau \\ IE University of Applied Sciences}

\begin{tabular}{|l|}
\hline Startseite \\
\hline Kooperation \\
\hline TFH Wildau \\
\hline Studiengänge \\
\hline Studieren in Wildau \\
\hline Leben in wildau \\
\hline $\begin{array}{l}\text { Arbeitsmöglichkeiten } \\
\text { und Stipendien }\end{array}$ \\
\hline Materialien \\
\hline Veröffentlichungen \\
\hline Bilder von Wildau \\
\hline Da kommen wir her \\
\hline Ansprechpartner \\
\hline \\
\hline Suche \\
\hline Impressum \\
\hline Sitemap \\
\hline
\end{tabular}

"Startseite " Einrichtungen " TQM " Lateinamerika

Eateinamerikanische Studenten an der TFH Wildau

Die Technische Fachhochschule Wildau heute

Die Technische Fachhochschule Wildau hat sich seit ihrer Gründung im Jahr 1991 zu einem wichtigen Wissenschaftszentrum und Wirtschaftsfaktor in der Hauptstadtregion BerlinBrandenburg entwickelt.

Zum Beginn des Wintersemesters 2005/2006 waren an der größten Fachhochschule des Landes Brandenburg 212 Mitarbeiter beschäftigt, darunter 62 Professoren. Zusätzlich wurden im Jahresdurchschnitt 53 Mitarbeiter aus Drittmittelforschungsprojekten finanziert.

Die TFH Wildau versteht sich in erster Linie als ein Dienstleistungsunternehmen. Dabei sind die wichtigsten Bereiche ihrer Tätigkeit:

- Ausbildung

- Angewandte Forschung und Entwicklung

- Weiterbildung

- Wissens- und Technologietransfer

- Förderung und Unterstützung von Existenzgründungen

- Internationale Zusammenarbeit

- Integration in die Region

- Kulturförderung

Die TFH Wildau ist eine praxisverbundene Hochschule und ein wichtiger Standortfaktor der Region. Sie zeichnet sich durch eine außerordentlich enge Verbindung zur Wirtschaft sowie zu Behörden und Verwaltungen aus. Sichtbares Zeichen dafür ist das hohe Volumen an angewandten Forschungs- und Entwicklungsaufträgen, die alljährlich und konstant durch Professoren, Mitarbeiter und Studenten der Hochschule eingeworben werden. Dies führt u. a. auch zu sehr guten und unmittelbaren Verbindungen zu Praxiseinrichtungen, die für die Qualität der Ausbildung von ausschlaggebender Bedeutung sind. Kulturförderung

Wichtige Kompetenzbereiche sind für uns:

- Neue Technologien

- Neue Materialien

- Umweltschutz

- Maschinenbau

- Verfahrenstechnik

- Biosystemtechnik und Bioinformatik

- Energiewirtschaft

- Logistik (Verkehrs- und Unternehmenslogistik)

- Informatik (Wirtschaftsinformatik, Software Engineering, Multimedia)

- Telematik (Telematikanwendungen u. a. in der Verkehrslogistik)

- Luftfahrttechnik und Luftfahrtlogistik

- Lasertechnik

- Oberflachentechnik

- Qualitätsmanagement

- Betriebswirtschaft und regionale Innovationsforschung

- Internettechnologien

- Interkulturalitat

- Verwaltungsmodernisierung

Die TFH Wildau geht auch weitere innovative Schritte, um ihr Profil als ein Zentrum der akademischen Weiterbildung zu stärken und icre internationale Attraktivitat zu erhöhen. Am 21. Februar 2005 wurde dazu al Graduate School das Wildas Institute of Technologr 21. (WIT) 200 wite . (WIT) gegrundet. Im Mittelpunkt des deutsch-und englischsprachigen Angebotes des WIT Ingenieure und Naturwissenschaftler aus dem In- und Ausland, insbesondere an Manager und Nachwuchskrafte sowohl in internationalen Großkonzernen als auch in wachstumsorientierten kleinen und mittleren Unternehmen. Darüber hinaus richten sich die Angebote zur interdisziplinären Weiterbildung bzw. zur Erzielung eines zweiten Berufsabschlusses auch an Akademiker, die eigene Unternehmensgründungsprojekte verfolgen. 
Lateinamerikanische Studenten an der TFH Wildau $\rightarrow$ Studiengänge

Schnelleinstieg für: Studierende | Mitarbeiter | Bewerber | Gäste | Wirtschaft |

\section{Technische Fachhochschule Wildau \\ University of Applied Sciences}

\begin{tabular}{|l}
\hline Startseite \\
\hline Kooperation \\
\hline TFH Wildau \\
\hline Studiengänge \\
\hline Studieren in Wildau \\
\hline Leben in Wildau \\
\hline Arbeitsmöglichkeiten \\
und Stipendien \\
\hline Materialien \\
\hline Veröffentlichungen \\
\hline Bilder von Wildau \\
\hline Da kommen wir her \\
\hline Ansprechpartner \\
\hline \\
\hline Suche \\
\hline Impressum \\
\hline Sitemap \\
\hline
\end{tabular}

" Startseite " Einrichtungen " TQM " Lateinamerika

= Lateinamerikanische Studenten an der TFH Wildau

E Studiengänge

- Bachelor Studiengänge

- Master Studiengänge

Letzte Anderung: 30.05.2008 12:20

Verantwortlich für diese Seite:

(C) 2008 Technische Fachhochschule Wildau 


\section{Lateinamerikanische Studenten an der TFH Wildau $\rightarrow$ Bachelor Studiengänge $\rightarrow$ Wirtschaftsinformatik}

Schnelleinstieg für: Studierende | Mitarbeiter | Bewerber | Gäste | Wirtschaft

\begin{tabular}{|l|l|}
\hline Startseite \\
\hline Kooperation \\
\hline TFH wildau \\
\hline Studiengänge \\
\hline Studieren in wildau \\
\hline Leben in wildau \\
\hline $\begin{array}{l}\text { Arbeitsmöglichkeiten } \\
\text { und Stipendien }\end{array}$ \\
\hline Materialien \\
\hline Veröffentlichungen \\
\hline Bilder von wildau \\
\hline Da kommen wir her \\
\hline Ansprechpartner \\
\hline
\end{tabular}

E Lateinamerikanische Studenten an der TFH Wildau

Bachelor-Studiengang Wirtschaftsinformatik

Der Bachelor-Studiengang Wirtschaftsinformatik vemittelt neben Fachwissen

Schlüsselqualifikationen und Methodenkompetenz grundlegende Kenntnisse über und

Verständnis für quantifizierbare Prozesse, wie z. B. Ressourcenplanung, Produktions- und Dienstleistungsplanung, Supply-Chain-Management, Investitionsprozesse, Absatz-, Umsatzund Deckungsbeitragsplanung, Investitionsprozesse, Tourenplanung, dienstleistungsorientierte Marketingplanung.

\section{Ausbildungsziele}

In allen Wirtschaftsbereichen nimmt die IT-gestützte (insbesondere die Internet-gestützte Abwicklung von Geschäftsprozessen zu. Die Beherrschung der Informatiksysteme ist eine zukünftige Hauptaufgabe der Unternehmen. Nachgefragt werden daher Integrationsfachleute, die sich sowohl mit relevanten Geschäftsprozessen als auch mit den technischen Grundlagen auskennen und die Fähigkeit besitzen, Prozesse zu analysieren, sie entsprechend zu modellieren und die Ergebnisse zu interpretieren. Der Studiengang Wirtschaftsinformatik (B.Sc.) orientiert sich an diesem Bedarf. Die Absolventen verfügen einerseits über fundierte wirtschafts- und informationstechnische Kenntnisse, andererseits über Fertigkeiten in der Geschaftsprozessmodellierung, die entscheidend zur erfolgreichen Bewaltigung in der betriebswirczessmodellierung, die entscheidend zur efolgreichen Bewaltigung modern interdisziplinares fachliches Know-how, $u$ a in Projektmanaement, und durch soziale Kompetenz auszeichnen.

\section{Einsatzgebiete/Berufsfelder}

Unabhängig von der Stagnation oder Entwicklung des Marktes sind Wirtschaftsinformatiker an der Schnittstelle der Wissenschaftsdisziplinen Wirtschaft und Informatik immer gefragt. Sie ermoglichen und sichern integrative, fachubergreifende Losungen und systematisches, strategisches und erfolgsorientiertes Handeln. Großunternehmen schätzen vor allem Fähigkeiten wie Systemdenken und Prozessgestaltung, KMU vor allem Innovationskompetenz aufgrund des aktuellen Wissens. Praxisorientierte Fachkräfte, die über derartige Kompetenzen verfügen, sind für Unternehmen risikoarm und häufig nach nur kurzer Einarbeitungszeit einsetzbar. Konkret eröffnet der Studiengang Wirtschaftsinformatik (B.Sc.) berufliche Perspektiven in

- Produktions- und Dienstleistungsunternehmen der Privatwirtschaft,

- Unternehmensberatungen,

- Banken,

- Kommunen, Landkreisen, Landes- oder Bundesbehörden sowie

- Verbanden.

\section{Studienaufbau}

Der Bachelor-Studiengang Wirtschaftsinformatik erstreckt sich über sechs Studiensemester, von denen die ersten vier ausschließlich in Form von Lehrveranstaltungen erfolgen und das fünfte Semester zusatzlich ein zwolfwochiges Praktikum beinhaltet. Im sechsten Semester liegt der Fokus auf der Erarbeitung der Bachelor-Thesis. Hierfür sind maximal 12 Wochen vorgesehen, wobei auch weiterhin Lehrveranstaltungen stattfinden.

\section{Studieninhalte}

Die Studieninhalte sind zusammengefasst in den Modulgruppen Informatik,

Wirtschfsinformatik, Betriabswirschaftere Sof Skills, Praktikum, Bachelor-Thesis. 
Lateinamerikanische Studenten an der TFH Wildau $\rightarrow$ Master Studiengänge

Schnelleinstieg für: Studierende | Mitarbeiter | Bewerber | Gäste | Wirtschaft |

\section{Technische Fachhochschule Wildau \\ University of Applied Sciences}

\begin{tabular}{|l}
\hline Startseite \\
\hline Kooperation \\
\hline TFH Wildau \\
\hline Studiengänge \\
\hline Studieren in Wildau \\
\hline Leben in Wildau \\
\hline $\begin{array}{l}\text { Arbeitsmöglichkeiten } \\
\text { und Stipendien }\end{array}$ \\
\hline Materialien \\
\hline Veröffentlichungen \\
\hline Bilder von Wildau \\
\hline Da kommen wir her \\
\hline Ansprechpartner \\
\hline \\
\hline Suche \\
\hline Impressum \\
\hline Sitemap \\
\hline
\end{tabular}

" Startseite " Einrichtungen " TQM " Lateinamerika " Lateinamerika Studiengang Haupt

= Lateinamerikanische Studenten an der TFH Wildau

Easter Studiengänge

Fachbereich Betriebswirtschaft/Wirtschaftsinformatik

\begin{tabular}{|c|c|c|c|}
\hline Studiengang & Abschluss & Daver & \\
\hline Business Management (ab WS 2009) & Master of Arts (MA) & 2 Jahre & Details \\
\hline lirtschaftsinformatik (ab WS 2009) & Master of Science & 2 Jahre & Details \\
\hline
\end{tabular}

Wirtschaftsinformatik (ab WS 2009) (M.Sc.)

Fachbereich Ingenieurwesen/Wirtschaftsingenieurwesen

\begin{tabular}{llll}
\hline Studiengang & Absch/uss & Daver & Flyer \\
\hline Biosystemtechnik/Bioinformatik & $\begin{array}{l}\text { Master of Science } \\
\text { (M.Sc.) }\end{array}$ & 2 Jahre Details \\
\hline Luftfahrttechnik/Luftfahrtlogistik & $\begin{array}{l}\text { Master of Engineering } \\
\text { (M.Eng.) }\end{array}$ & 2 Jahre Details D \\
\hline $\begin{array}{l}\text { Technisches Management und Logisik (ab } \\
\text { WS 2009) }\end{array}$ & $\begin{array}{l}\text { Master of Engineering } \\
\text { (M.Eng.) }\end{array}$ & 2 Jahre Details \\
\hline Telematik & $\begin{array}{l}\text { Master of Engineering } \\
\text { (M.Eng.) }\end{array}$ & 2 Jahre Details D \\
\hline Photonics & $\begin{array}{l}\text { Master of Engineering } \\
\text { (M.Eng.) }\end{array}$ & $\begin{array}{c}1 \\
1 / 2 \text { Jahre Details D }\end{array}$
\end{tabular}

Fachbereich Wirtschaft, Verwaltung und Recht

\begin{tabular}{llll}
\hline Studiengang & Abschluss & Dauer & Flyer \\
\hline Europäisches Management & Master of Arts (MA) & 2 Jahre Details D \\
\hline Wirtschaft und Recht & Master of Laws (LLM.) & 2 Jahre Details \\
\hline
\end{tabular}

\begin{tabular}{|c|c|c|c|}
\hline \multicolumn{2}{|l|}{$<1$} & & $>$ \\
\hline Fertig & (9) Internet & $+100 \%$ & - \\
\hline
\end{tabular}


Lateinamerikanische Studenten an der TFH Wildau $\rightarrow$ Studieren in Wildau

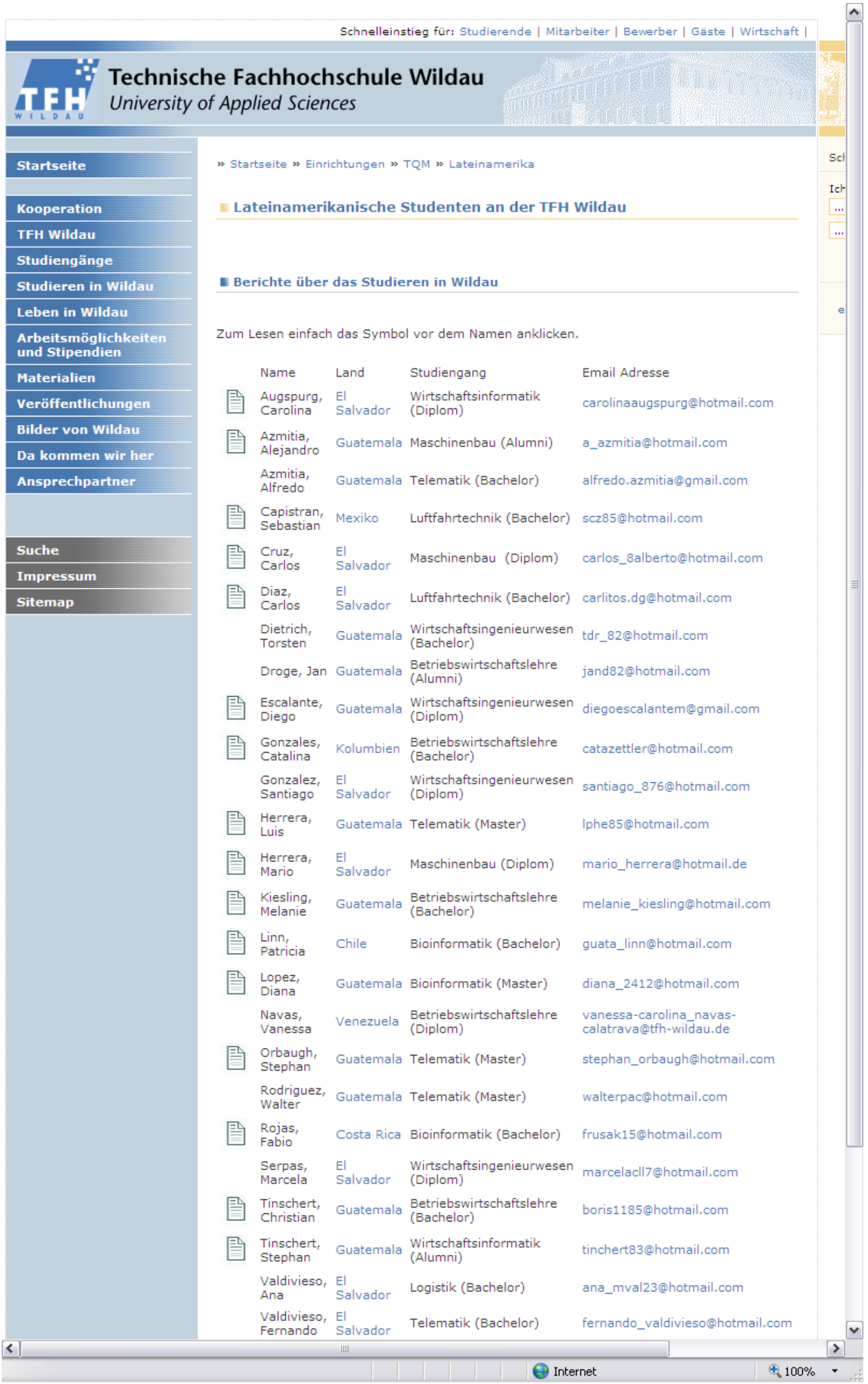


Lateinamerikanische Studenten an der TFH Wildau $\rightarrow$ Studieren in Wildau $\rightarrow$ C. Augspurg

Schnelleinstieg für: Studierende | Mitarbeiter | Bewerber | Gäste | Wirtschaft |

\section{Technische Fachhochschule Wildau \\ University of Applied Sciences}

\begin{tabular}{|l}
\hline Startseite \\
\hline Kooperation \\
\hline TFH wildau \\
\hline Studiengänge \\
\hline Studieren in Wildau \\
\hline Leben in wildau \\
\hline Arbeitsmöglichkeiten \\
und Stipendien \\
\hline Materialien \\
\hline Veröffentlichungen \\
\hline Bilder von Wildau \\
\hline Da kommen wir her \\
\hline Ansprechpartner \\
\hline Suche \\
\hline Impressum \\
\hline Sitemap \\
\hline
\end{tabular}

"Startseite " Einrichtungen " TQM " Lateinamerika " Studieren in Wildau

= Lateinamerikanische Studenten an der TFH Wildau

Earolina Augspurg

Name: Carolina Augspurg

Geburtsdatum: 30 März, 1986

Herkunft: El Salvador

Studiengang: Wirtschaftsinformatik

Email: caroaugspurg@yahoo.com

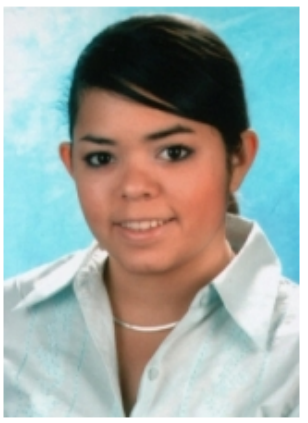

Mein Name ist Carolina Augspurg. Mein Bachillerato habe ich an der Deutschen Schule San Salvador absolviert. Seitdem studiere ich Wirtschaftsinformatik an der TFH-Wildau und befinde mich zurzeit im 6 . Semester.

Die Wirtschaftsinformatik ist eine relativ junge Wissenschaftsdisziplin. Der Studierende des Studiengangs Wirtschaftsinformatik soll befähigt werden, selbständig Aufgabenstellungen an der Schnittstelle zwischen Informatik und Betriebswirtschaftslehre zu lösen. Hierzu sind sowohl gründliche Kenntnisse der Informatik (z. B. Methoden der Systemanalyse und Optimierung) als auch der betriebswirtschaftlichen Anwendungsfelder ( $z$. B.

Produktionswirtschaft, Rechnungswesen, Controlling und Organisation) notwendig.

Das Leben in Wildau ist ruhig, aber sehr schön. Dank der verschiedenen Nationalitäten die man in den Wohnheimen findet, ist man ständig in Kontakt mit neuen Kulturen und Freunden. Einkaufs-und-Freizeitmöglichkeiten, sowie eine SBahn-Verbindung, mit der man in 20 min. Berlin erreichen kann, gehören zu den Vorzügen von Wildau.

Nach drei Jahren Leben in Deutschland und nur noch ein Jahr bis zum Diplom-Abschluss kann ich nur noch sagen, dass es die richtige Entscheidung war an der TFH-Wildau zu studieren.

Letzte Anderung: 05.06.2008 $18: 16$

Verantwortlich für diese Seite:

(C) 2008 Technische Fachhochschule Wildau 


\section{Lateinamerikanische Studenten an der TFH Wildau $\rightarrow$ Leben in Wildau}

Schnelleinstieg für: Studierende | Mitarbeiter | Beverber | Gaste | Wirtschaft |

\section{Technische Fachhochschule Wildau \\ IE University of Applied Sciences}

\begin{tabular}{|l|}
\hline Startseite \\
\hline Kooperation \\
\hline TFH Wildau \\
\hline Studiengänge \\
\hline Studieren in Wildau \\
\hline Leben in Wildau \\
\hline Arbeitsmöglichkeiten \\
und Stipendien \\
\hline Materialien \\
\hline Veröffentlichungen \\
\hline Bilder von Wildau \\
\hline Da kommen wir her \\
\hline Ansprechpartner \\
\hline \\
\hline Suche \\
\hline Impressum \\
\hline Sitemap \\
\hline
\end{tabular}

"Startseite " Einrichtungen " TQM " Lateinamerika

E Lateinamerikanische Studenten an der TFH Wildau

Eerichte über das Leben in Wildau und in Berlin

Leben in Wildau

Das Leben in Wildau lässt sich recht vielseitig gestalten. Mit einer Einwohnerzahl von knapp 10.000 und über 3.000 Studierenden ist die Gemeinde Wildau, gelegen im Bundesland Brandenburg, zwar überschaubar, profitiert allerdings enorm von einigen bemerkenswerten Randbedingungen. Dazu gehört in erster Linie die Nähe zur deutschen Hauptstadt Berlin. Allerdings behält der Ort durch seine Einbettung in die wunderschöne Landschaft Südbrandenburgs seinen ländlichen Charme. Somit sind zahlreiche Möglichkeiten gegeben, Sudbranden einerseits kurzfristige und nicht sehr zeitaufwendige

Ein wirklicher Ortskern existiert in der Gemeinde Wildau momentan noch nicht. Dies ist dem regional bekannten und stark besuchten Einkaufszentrum A-10-Center geschuldet. Der wie der Name schon verrat, unmittelbar an der Autobahnabfahrt Wildau des südlichen Berliner Rings oder auch BAB 10 gelegen ist. Eigentlich ist die Gegend um die TFH Wildau / S-Bahnhof der ursprüngliche Ortsmittelpunkt. Es wird allerdings stark an einer Erneuerung gearbeitet. Die baulichen Maßnahmen sind weitestgehend abgeschlossen und immer mehr füllen sich die zahlreichen Geschafte und Restaurants / Bars mit Leben.

Neben den Freizeitmöglichkeiten im Freien existieren zahlreiche Gelegenheiten, die auch bei schlechtem Wetter keinen Grund zum Trübsal-Blasen geben. Als erstes ist da die Schwimmhalle, deren Wiedereröffnung nach umfangreichen Um- und Ausbauarbeiten erst vor kurzem stattgefunden hat, zu nennen. Dort werden nun auch umfangreiche Kurse aus den Bereichen Wellness und Fitness angeboten. Unweit von der Schwimmhalle befindet sich ein Fußballplatz, auf dem unter anderem alljährlich das Fußballturnier der TFH Wildau ausgetragen wird. Auf dem Gelände des A-10-Centers befindet sich eine Erlebniswelt mit einem Kino, einem Bowling-Center, einem Billard-Center als auch verschiedenen Bars und Restaurants.

Der Ort verfügt über eine überdurchschnittliche Infrastruktur. Einerseits ist ein direkter Anschluss an die BAB 10 gegeben, andererseits ist der Ort auch an den S-Bahn-Verbund Berlins angeschlossen. Im Ort stellt die dezentrale Lage der einzelnen Freizeitmöglichkeiten aufgrund einer guten Verkehrsanbindung mit dem Bus kein Problem dar.

Diana Lopez

\section{Leben in Berlin}

Als Hauptstadt von Deutschland bietet Berlin verschiedene Sehenswürdigkeiten. Wahrzeichen der Stadt ist der Fernsehturm, von dessen Panoramakaffee man in aller Ruhe die wunderschöne Aussicht auf ganz Berlin bewundern kann. Mit seinen 368 Metern Höhe ist er zugleich das höchste Gebaude der Stadt und Zentrum des Alexanderplatzes. In unmittelbarer zugleich das hochste Gebaude der Stadt und Zentrum des Alexanderplatzes. In unmittelbare Nahe zum "Großen" befindet sich der Sitz des Berliner Burgermeisters das Rote Rathaus.
seiner rot verklinkerten Fassade bildet es einen beeindruckenden Kontrast zum dahinter seiner rot verklinkerten Fassade bildet es einen beeindruckenden Kontrast zum dahinter
liegenden Nikolaiviertel, dass mit seinen vielen Geschaften, Restaurants, Cafes und Museen liegenden Nikolaiviertel, dass mit seinen
zu einem schönen Spaziergang einlädt.

Der Alexanderplatz selbst bietet sich als Ausgangspunkt für viele Spaziergänge durch Berlin an. In Richtung Unter den Linden" passiert man den Berliner Dom im Stiel der italienischen Hochrenaissance und Barock aus dem 19. Jahrhunder, die Marchenbrücke, die Staatsoper, die Humbolt-Universitat und zu guter letzt das Brandenburgertor. Hinter dem Brandenburgertor am Ende der Straße des 17.Juni's erhebt sich majestatisch auf der Siegessaule die "Gold am Ende der Strabe des 17.Juni's erhebt sich majestatisch auf der Siegessaule die "Gold Deutschland, der Reichstag mit seiner begehbaren Glaskuppel und das Bundeskanzleramt. Deutschland, der Reichstag mit seiner begehbaren Glaskuppel und das Bundeskanzleramt.
Bedeutend in der Geschichte Berlins und auch immer einen Besuch wert, sind die ehemalige Mauer von Berlin und der Checkpoint Charlie am Osthafen. Eine der bekanntesten Shoppingmailen Berlins ist der Kudamm. In dessen Mitte befindet sich die nach den Weltkriegen restaurierte Gedächniskirche und das Europacenter. Gerade einen Steinwurf entfernt kann man im Zoologischen Garten sowie Im Aquarium Tiere aus verschiedenen Regionen der Welt bestaunen. Eine Alternative hierzu, ist der im Osten der Stadt gelegene Tierpark. Mit seinen weitreichenden Freigehegen zählt der von Dr. Heinrich Dathe gegründete Park zu den größten seiner Art in Europa. Alle Sportbegeisterten sollten in Berlin unter keinen Umständen eine der verschiedenen Sportveranstaltungen im Olympiastadion, der Max Schmeling - Halle und dem Velodrom verpassen. Weitere kulturelle Eindrücke sammelt man auch bei einem Besuch der zahlreichen Schlösser und Museen von Berlin. 


\section{Lateinamerikanische Studenten an der TFH Wildau $\rightarrow$ Arbeitsmöglichkeiten und Stipendien}

Schnelleinstieg für: Studierende | Mitarbeiter | Bewerber | Gäste | Wirtschaft |

\section{Technische Fachhochschule Wildau \\ University of Applied Sciences}

Startseite

Kooperation

TFH Wildau

Studiengänge

Studieren in Wildat

Leben in wildau

Arbeitsmöglichkeiten und Stipendien

Materialien

Veröffentlichungen

Bilder von Wildau

Da kommen wir her

Ansprechpartner

\section{Suche}

Impressum

Sitemap
"Startseite " Einrichtungen " TQM " Lateinamerika

Eateinamerikanische Studenten an der TFH Wildau

E Arbeitsmöglichkeiten und Stipendien

Studentische Hilfskraft

Als studentische Hilfskraft bezeichnet man Studierende, die Professoren oder Dozenten in verschiedenen Tätigkeiten unterstützen.

Kontakte zur Besetzung studentischer Hilfskraftstellen ergeben sich oft in Seminaren oder Vorlesungen, einige werden auch ausgeschrieben (Stellenausschreibungen Studentische Mitarbeiter) oder per Rundmail angeboten. Hat man einen interessanten Job entdeckt, entweder durch einen Professor, Email oder Stellenanzeige, ist der nächste Schritt, sein Bewerbungsunterlagen zu erstellen. Wird man für die Stelle angenommen, muss nur noch ein Arbeitsvertrag unterschrieben werden.

In der Regel darf man ein Maximum von 20 Stunden pro Woche arbeiten mit einem

Stundenlohn von $4,97 € / h$ (alt) bzw. 5,11€/h (ab 01.05.08). Einige der lateinamerikanischen Studenten sind als studentische Hilfskraft in der Bibliothek oder beim Hochschulrechenzentrum (HRZ) tätig und berichten nur positives darüber.

\section{Stipendium}

Es gibt die Möglichkeit, an der TFH-Wildau ein Stipendium aufgrund besonders guter Studienleistungen zu erhalten. Annahmekriterien und wichtige Unterlagen, um ein Stipendium zu bekommen, sind:

- Gute/sehr gute Leistungen

- Anschreiben/Motivationsbrief

- Empfehlungen durch einen Professor und durch den Dekan

Wer näheren Informationen über das Thema wissen möchte, kann sich bei der Hochschulverwaltung erkundigen.

\section{Praktikum}

Das Praktikum ist als praktische Ergänzung zur theoretischen Ausbildung in Unternehmen und Organisationen gedacht.

Im Internet, in Fachzeitschriften oder auf Messen werden Praktikumsstellen angeboten. Interessiert man sich für einen bestimmten Job, schickt man die Bewerbungsunterlagen zu der jeweiligen Firma. Hat die Firma wiederum Interesse, wird man zu einem

derstellungs diese Firma eingestellt.

Ein Praktikum dauert in der Regel 3 bis 6 Monate. Die Vergütung variiert von Unternehmen zu Unternehmen.

Nebenjob

Ein Nebenjob kann Tätigkeiten unterschiedlicher Art bedeuten, von Babysitten über Nachhilfe geben bis hin zum Zeitungsaustragen oder Kellnern. Außerdem kann ein Nebenjob auch zeitlich befristet sein, wenn es sich beispielsweise um eine Saisonarbeit im Winter oder Sommer handelt oder ein Ferienjob.

Ausländische Studenten dürfen gemäß §16 Abs. 3 Aufenhaltsgesetz bis 90 ganzen oder 180 halben Tagen im Jahr ohne Arbeitserlaubnis in Deutschland arbeiten.

Die Vergütung einer Nebentätigkeit hängt meistens von der Arbeitsintensität und der Art der Tatigkeit $\mathrm{ab}$.

Fördermöglichkeiten für Deutsche bzw. für Ausländer 
Lateinamerikanische Studenten an der TFH Wildau $\rightarrow$ Materialien

Schnelleinstieg für: Studierende | Mitarbeiter | Bewerber | Gäste | Wirtschaft |

\section{Technische Fachhochschule Wildau \\ University of Applied Sciences}

\begin{tabular}{|l}
\hline Startseite \\
\hline Kooperation \\
\hline TFH Wildau \\
\hline Studiengänge \\
\hline Studieren in Wildau \\
\hline Leben in Wildau \\
\hline $\begin{array}{l}\text { Arbeitsmöglichkeiten } \\
\text { und Stipendien }\end{array}$ \\
\hline Materialien \\
\hline Veröffentlichungen \\
\hline Bilder von Wildau \\
\hline Da kommen wir her \\
\hline Ansprechpartner \\
\hline \\
\hline Suche \\
\hline Impressum \\
\hline Sitemap \\
\hline
\end{tabular}

"Startseite " Einrichtungen " TQM " Lateinamerika

E Lateinamerikanische Studenten an der TFH Wildau

Elyer und Poster

Flyer

Fachbereich Betriebswirtschaft/Wirtschaftsinformatik

- Betriebswirtschaft.pdf

- Wirtschaftsinformatik.pdf

Fachbereich Ingenieurwesen/Wirtschafsingiurwesen

- Maschinenbau.pdf

- PhysikalischeTechnik.pdf

- Verfahrenstechnik.pdf

- Logistik.pdf

- Wirtschaftsingenieur.pdf

- Biosystemtechnik Bachelor.pdf

- Biosystemtechnik Master.pdf

- Luftfahrttechnik.pdf

- Telematik Bachelor.pdf

- Telematik Master.pdf

- Photonics.pdf

Fachbereich Wirtschaft, Verwaltung und Recht

- Verwaltung und Recht.pdf

- Wirtschaft und Recht.pdf

- Europäisches Management Bachelor.pd

- Europaisches Management Master.pd

Poster

- Studium 2007 auf deutsch.pdf

- Studium 2007 auf englisch.pdf

Zum Studienführer

Letzte Änderung: 29.05.2008 17:03

Verantwortlich für diese Seite:

(C) 2008 Technische Fachhochschule Wildau 
Lateinamerikanische Studenten an der TFH Wildau $\rightarrow$ Veröffentlichungen

Schnelleinstieg für: Studierende | Mitarbeiter | Bewerber | Gäste | Wirtschaft |

\section{Technische Fachhochschule Wildau \\ University of Applied Sciences}

\begin{tabular}{|l}
\hline Startseite \\
\hline Kooperation \\
\hline TFH Wildau \\
\hline Studiengänge \\
\hline Studieren in Wildau \\
\hline Leben in Wildau \\
\hline $\begin{array}{l}\text { Arbeitsmöglichkeiten } \\
\text { und Stipendien }\end{array}$ \\
\hline Materialien \\
\hline Veröffentlichungen \\
\hline Bilder von Wildau \\
\hline Da kommen wir her \\
\hline Ansprechpartner \\
\hline \\
\hline Suche \\
\hline Impressum \\
\hline Sitemap \\
\hline
\end{tabular}

"Startseite " Einrichtungen " TQM " Lateinamerika

Eateinamerikanische Studenten an der TFH Wildau

E Veröffentlichungen

- Jahrbuch Bericht für die DS San Salvador

- Präsentation für die Schüler aus der DS Guatemala

- Bilder vom Besuch von Thomas Castella

- Videopräsentationen der TFH Wildau

Letzte Änderung: 21.05.2008 16:24

Verantwortlich für diese Seite:

(C) 2008 Technische Fachhochschule Wildau 
Lateinamerikanische Studenten an der TFH Wildau $\rightarrow$ Veröffentlichungen $\rightarrow$ Jahrbuchbericht für die DS San Salvador

Schnelleinstieg für: Studierende | Mitarbeiter | Bewerber | Gäste | Wirtschaft |

\begin{tabular}{l}
\hline Technische Fachhochschule Wildau \\
University of Applied Sciences \\
\hline Startseite \\
\hline Kooperation \\
\hline TFH wildau \\
\hline Studiengänge \\
\hline Studieren in wildau \\
\hline
\end{tabular}

Leben in Wildau

Arbeitsmöglichkeiten und Stipendien

Materialien

Veröffentlichungen

Bilder von wildau

Da kommen wir her

Ansprechpartner

"Die Sammlung von Anekdoten und Geschichten ist der größte Schatz der Menschheit" Goethe.

Der Schnee ist schon geschmolzen, die Bäume blühen wieder und lassen den Weg, der sonst so lang erscheint in neuem Glanz erstrahlen. Die Wintersachen sind im Schrank verstaut. Statt des morgenltichen überpünktlichen Buses sind wir auf die Fussvariante umgestiegen. Die Chancen den Anschlusszug zu bekommen stehen um einiges höher und die $20 \mathrm{~min}$ Wartezeit kommen uns nicht mehr so lange vor. Der Frühling ist da und endlich gehört auch unser Herz zu Wildau und der Fachhochschule, die uns mit offenen Armen empfangen hat.

Die TFH Wildau hat ihre Pforten geöffnet, bessonders für Ausländer, eine Fachhochschule die uns zuhört und die unsere Wünsche auch in die Tat umsetzt. Unseren Dozenten und der Inhalt des Studiums sind auf sehr hohem Niveau, wie auch die gut ausgestatteten Laboratorien. Dies lässt uns an eine Zeit zurück denken, in der wir geträumt haben in Deutschland zu studieren. Nicht nur der Mathematik- und Physik-unterricht bestatigen uns, dass die Vorbereitung durch dicht nur der Mathemati-undern auch das altägliche die Schule wertvoll war, sondern auch das altagliche Leben birgt Herausforderungen, die wir uns wichtige Werte und Normen vermittelt, die uns helfen in einer völlig anderen Kultur zurecht zu kommen.

Wir beginnen jetzt mit dem zweiten Semester, wenn wir mal zurück schauen zu unserem erstenTag an der Fachhochschule, dann war es für die meisten Deutschen wohl nur ein verregneter Septembertag, doch fur uns war es der Anfang eines Traumes. Aber auch einer guten Freundschaft zwischen drei Salvadorianern, die sich manchmal treffen um Pupusas zu braten und sich Geschichten aus den unvergeslichen Schuljahren zu erzählen, die trotz des Witzes, mit dem sie erzählt werden, nur der Wahrheit entsprechen. Trotz der großen Entfernung zu unseren Familien, Freunden, traumhaftem Heimatland und angenehmen Klima, ist es unserer Traum, den wir jetzt leben können.

Wildau ist eine ruhige Stadt, manchmal etwas zu ruhig, obwohl die drei Studentenwohnheime, die Partys und das Studium die beste Mischung ergeben. Komellitoren und Freuden aus die Partys und das Studium die beste Mischung ergeben. Komelitoren und Freuden aus verschiedenen Nationalitaten wie zB. aus Guatemala, Polen, Russland und Indien bilden diese aus ganz Deutschland stammenden Freunde vergessen, bessonders die aus Berlin, die uns mit ihrem Akzent und ihrer Art die Uhrzeit zu sagen die ersten Tage zum verzweifeln brachten.

Wir liebten Deutschland auf den ersten Blick, Bier und Wurst, in guten wie in schlechten Zeiten....

Carlos, Carolina und Fernando
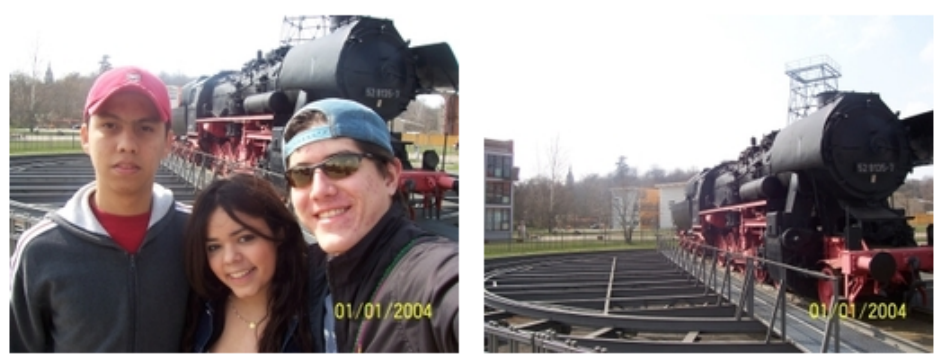
Lateinamerikanische Studenten an der TFH Wildau $\rightarrow$ Veröffentlichungen $\rightarrow$ Bilder vom Besuch von Thomas Castella

Schnelleinstieg für: Studierende | Mitarbeiter | Bewerber | Gäste | Wirtschaft |

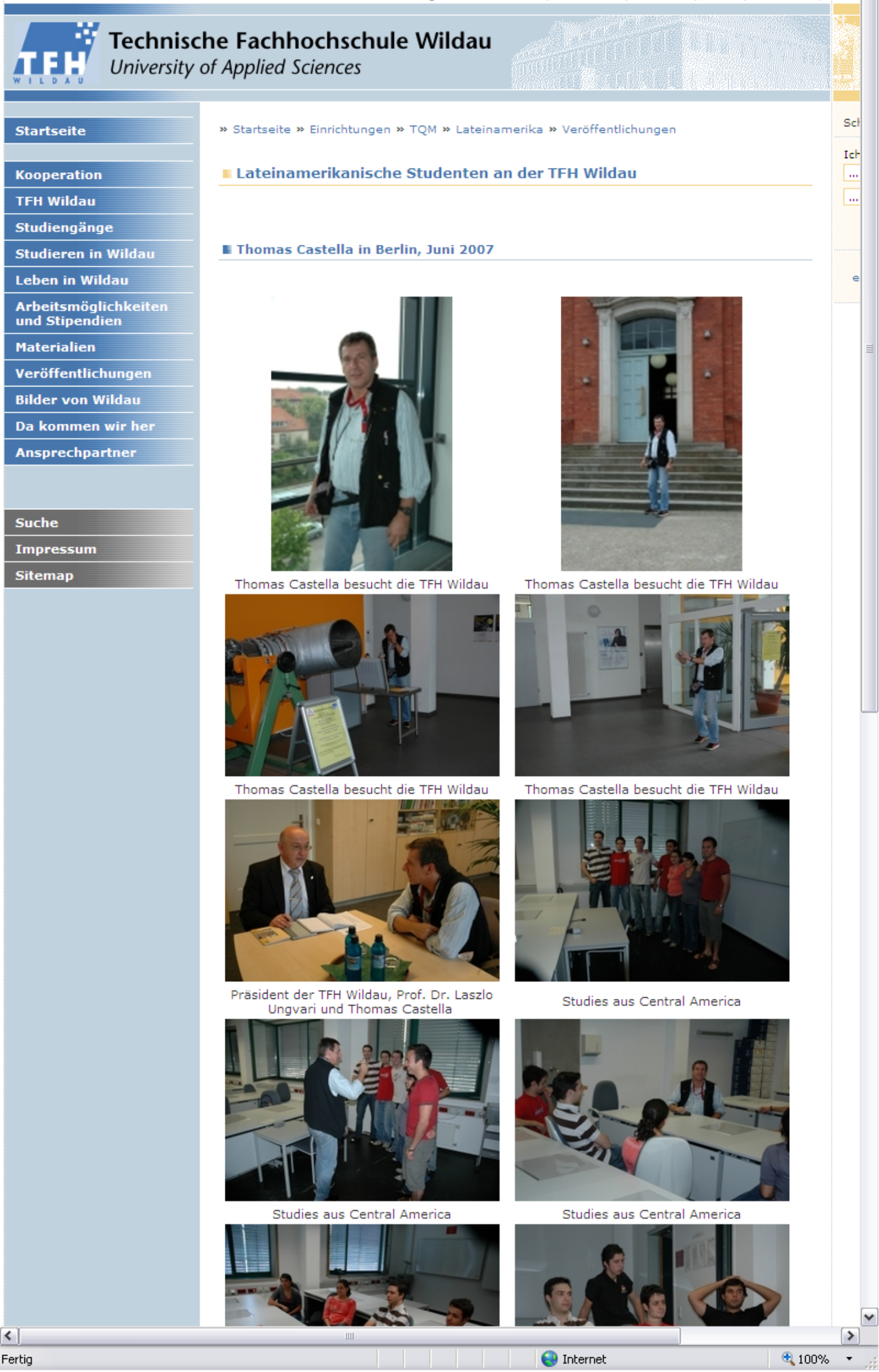


Lateinamerikanische Studenten an der TFH Wildau $\rightarrow$ Bilder von Wildau

Schnelleinstieg für: Studierende | Mitarbeiter | Bewerber | Gäste | Wirtschaft |

\section{Technische Fachhochschule Wildau \\ University of Applied Sciences}

\begin{tabular}{|l}
\hline Startseite \\
\hline Kooperation \\
\hline TFH Wildau \\
\hline Studiengänge \\
\hline Studieren in Wildau \\
\hline Leben in Wildau \\
\hline Arbeitsmöglichkeiten \\
und Stipendien \\
\hline Materialien \\
\hline Veröffentlichungen \\
\hline Bilder von Wildau \\
\hline Da kommen wir her \\
\hline Ansprechpartner \\
\hline \\
\hline Suche \\
\hline Impressum \\
\hline Sitemap \\
\hline
\end{tabular}

"Startseite " Einrichtungen " TQM " Lateinamerika

Eateinamerikanische Studenten an der TFH Wildau

Bilder von Wildau und Umgebung

- Wildau

- Wohnheime

- Party

- Sehenswürdigkeiten in Berlin

- Campus

- Labore

Letzte Änderung: 16.05.2008 08:52 Verantwortlich für diese Seite:

(C) 2008 Technische Fachhochschule Wildau 
Lateinamerikanische Studenten an der TFH Wildau $\rightarrow$ Bilder von Wildau $\rightarrow$ Wildau

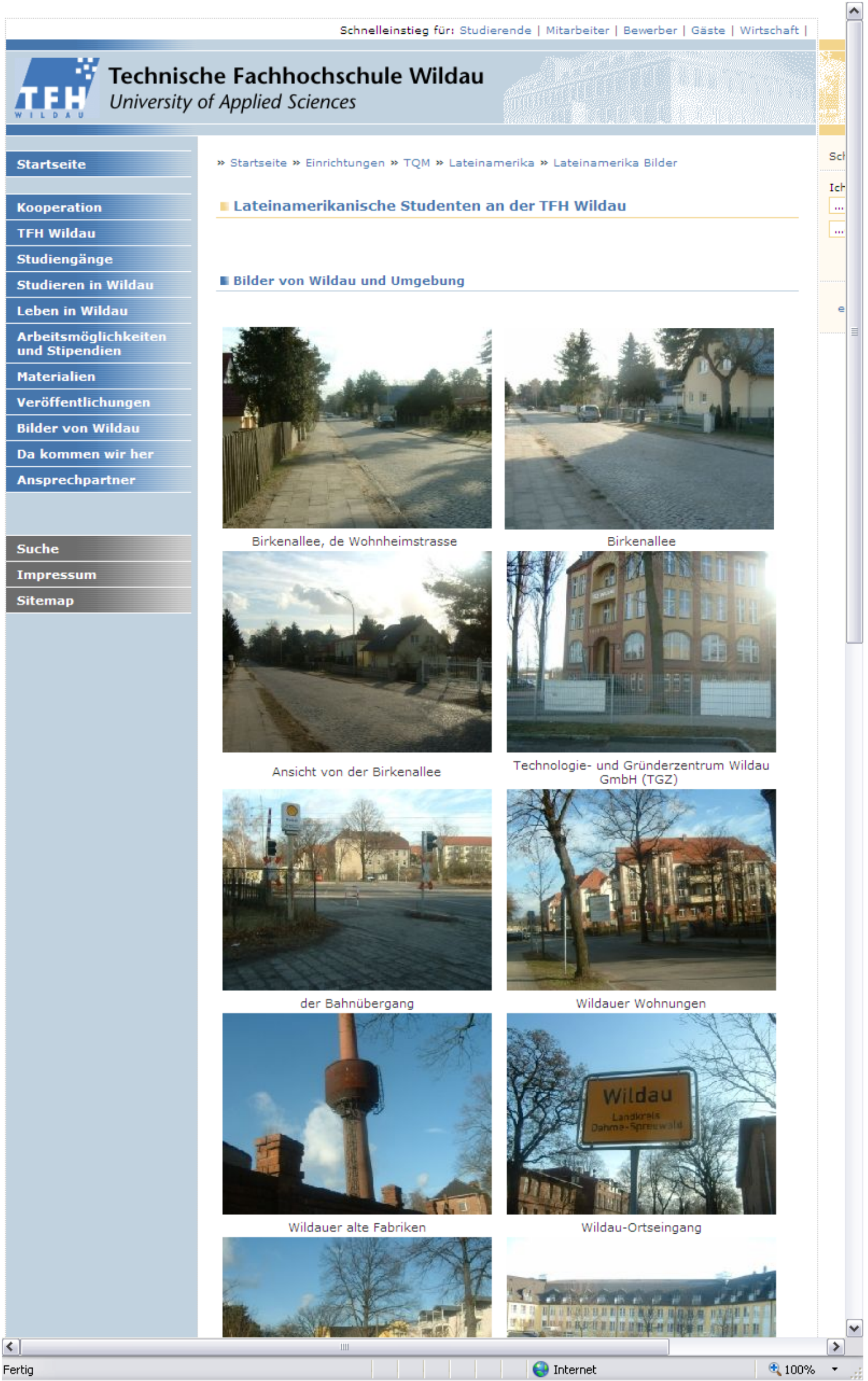


Lateinamerikanische Studenten an der TFH Wildau $\rightarrow$ Bilder von Wildau $\rightarrow$ Wohnheime

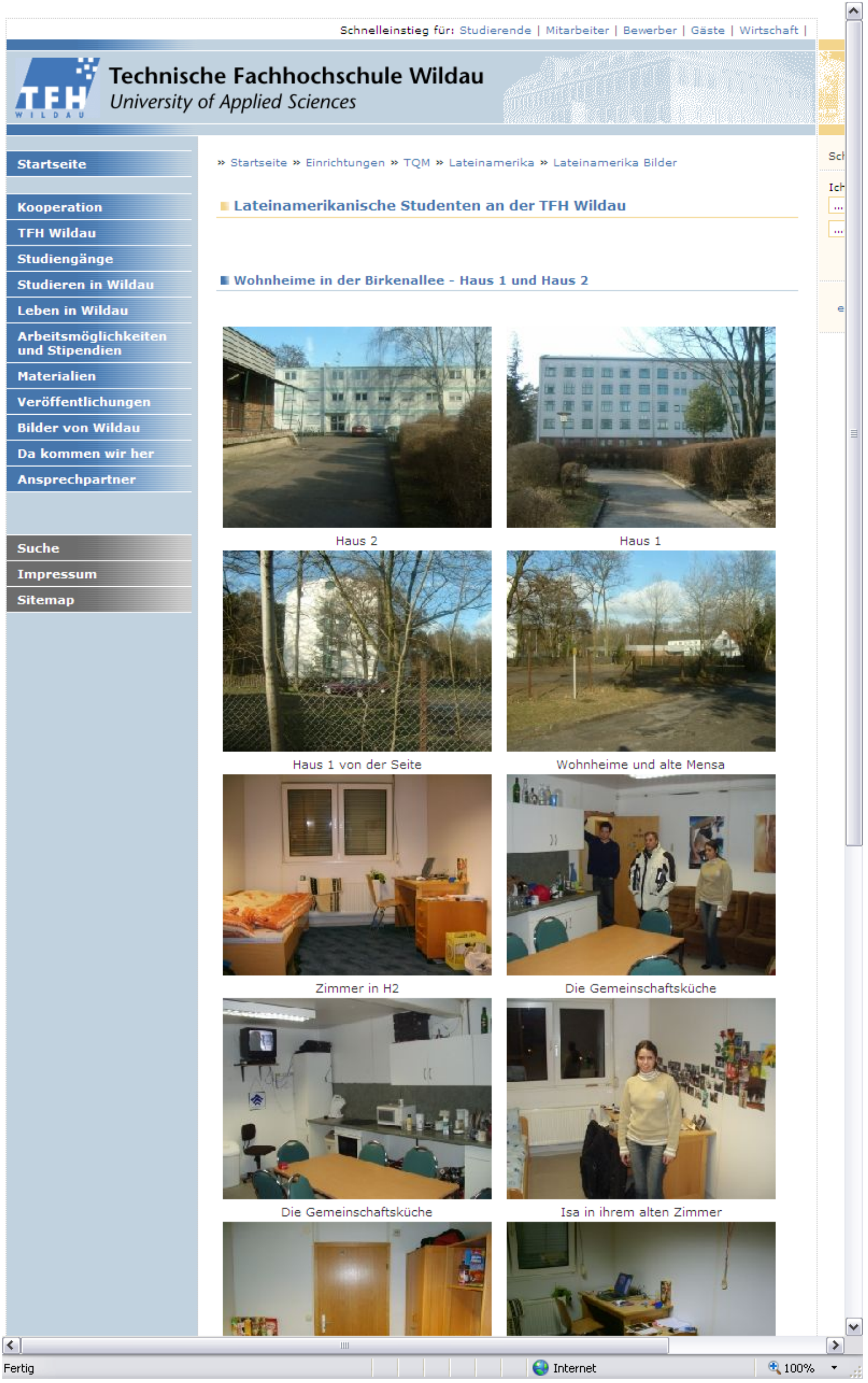


Lateinamerikanische Studenten an der TFH Wildau $\rightarrow$ Bilder von Wildau $\rightarrow$ Campus

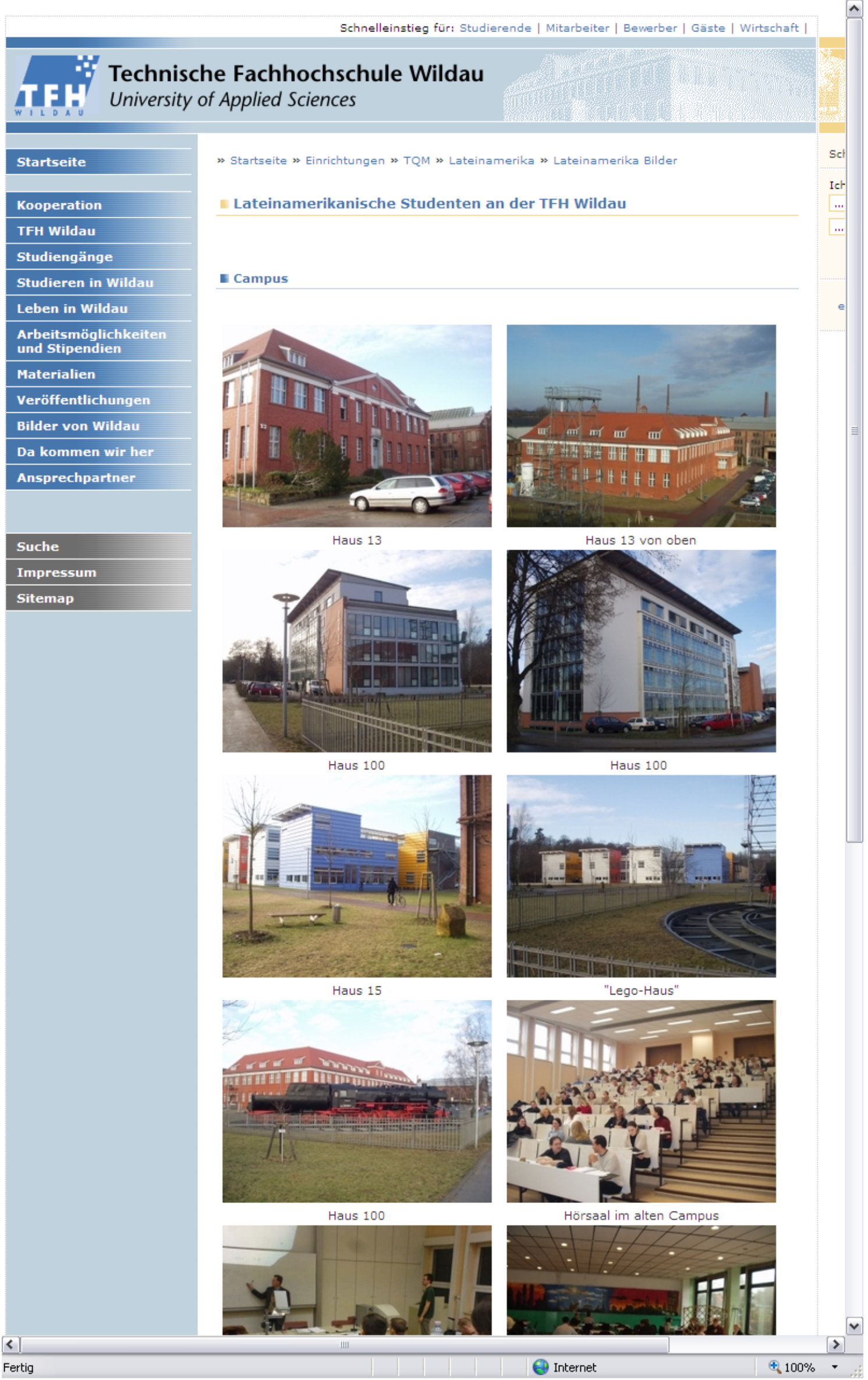


Lateinamerikanische Studenten an der TFH Wildau $\rightarrow$ Da kommen wir her

Schnelleinstieg für: Studierende | Mitarbeiter | Bewerber | Gäste | Wirtschaft |

\section{Technische Fachhochschule Wildau \\ University of Applied Sciences}

\begin{tabular}{|l}
\hline Startseite \\
\hline Kooperation \\
\hline TFH Wildau \\
\hline Studiengänge \\
\hline Studieren in Wildau \\
\hline Leben in Wildau \\
\hline Arbeitsmöglichkeiten \\
und Stipendien \\
\hline Materialien \\
\hline Veröffentlichungen \\
\hline Bilder von Wildau \\
\hline Da kommen wir her \\
\hline Ansprechpartner \\
\hline \\
\hline Suche \\
\hline Impressum \\
\hline Sitemap \\
\hline
\end{tabular}

"Startseite " Einrichtungen " TQM " Lateinamerika

Lateinamerikanische Studenten an der TFH Wildau

E Unsere Heimatländer

- Chile

- Costa Rica

- El Salvador

- Guatemala

- Kolumbien

- Mexiko

- Venezuela

Letzte Anderung: 20.05.2008 08:22

verantwortlich für diese Seite:

(C) 2008 Technische Fachhochschule Wildau 
Lateinamerikanische Studenten an der TFH Wildau $\rightarrow$ Da kommen wir her $\rightarrow$ El Salvador

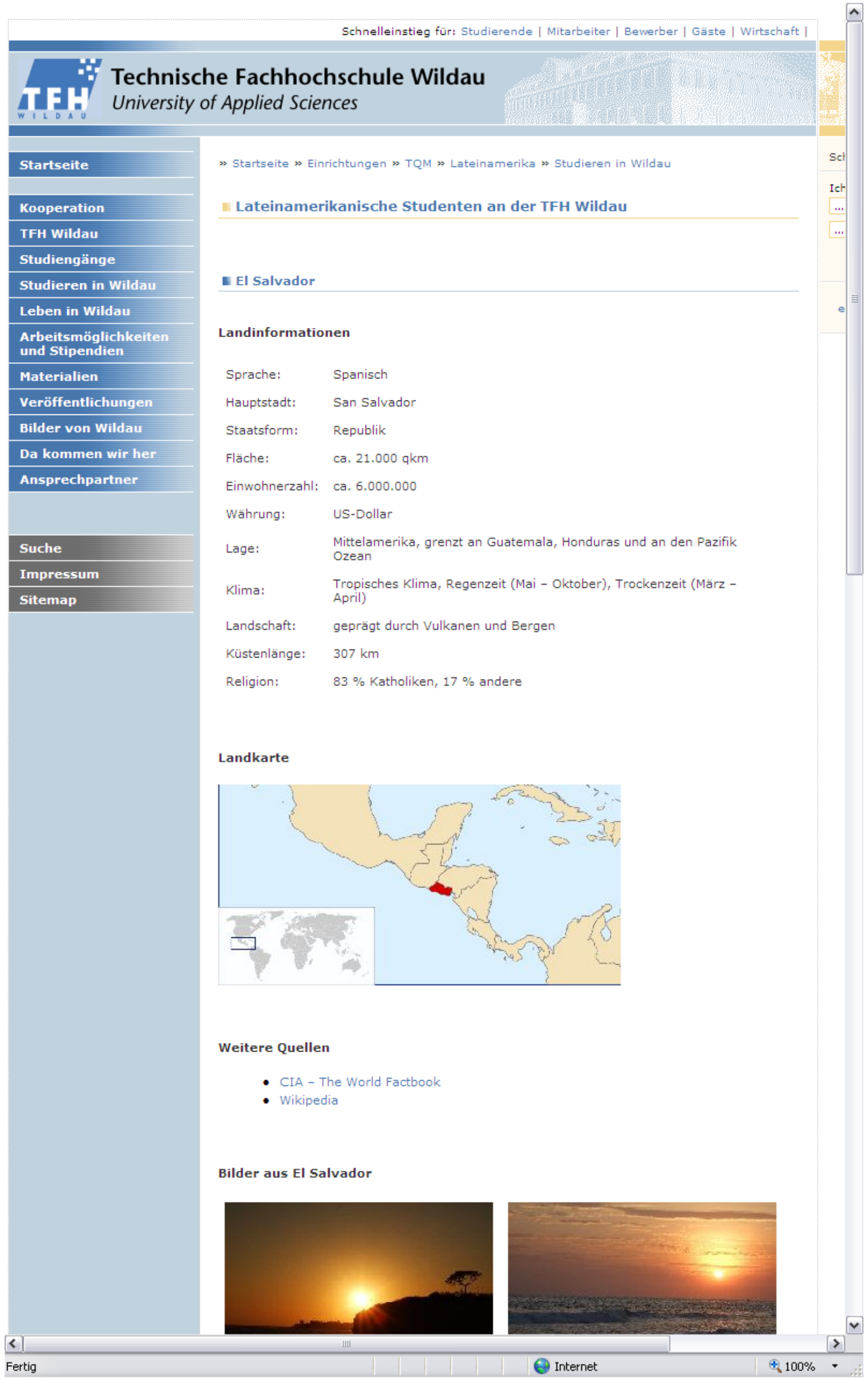


Lateinamerikanische Studenten an der TFH Wildau $\rightarrow$ Ansprechpartner

Schnelleinstieg für: Studierende | Mitarbeiter | Bewerber | Gäste | Wirtschaft |

\section{Technische Fachhochschule Wildau \\ I E University of Applied Sciences}

Startseite

Kooperation

TFH Wildau

Studiengänge

Studieren in Wildau

Leben in wildau

Arbeitsmöglichkeiten

und Stipendien

Materialien

Veröffentlichungen

Bilder von Wildau

Da kommen wir her

Ansprechpartner

\section{Suche}

Impressum

Sitemap
"Startseite " Einrichtungen " TQM " Lateinamerika

E Lateinamerikanische Studenten an der TFH Wildau

Ansprechpartner

Die Ansprechpartner für diese Kooperation:

- Prof. Dr. László Ungvári (Präsident der TFH-Wildau)

- Prof. Dr. Christian Müller

Die Schulleiter und Studienfachberater unserer Kooperationspartner:

- Deutschen Schule in Guatemala

- Deutschen Schule in El Salvador

- Deutschen Schule in Costa Rica

- Deutschen Schule in Cali, Kolumbier

etzte Änderung: 18.05.2008 17:58

Verantwortlich für diese Seite:

(C) 2008 Technische Fachhochschule Wildau 


\section{Selbständigkeitserklärung}

Hiermit erkläre ich, dass ich die vorliegende Arbeit selbständig verfasst, noch nicht anderweitig für Prüfungszwecke vorgelegt, keine anderen als die angegebenen Quellen oder Hilfsmittel verwendet, sowie wörtlich und sinngemäße Zitate als solche gekennzeichnet habe.

Ort, Datum, Unterschrift 